\title{
Bugle-96 Validation with Morse-SGC/S Using Water and Iron Experiments from Sinbad 97
}

by

A. Blanchard

Westinghouse Savannah River Company

Savannah River Site

Aiken, South Carolina 29808

D. Biswas

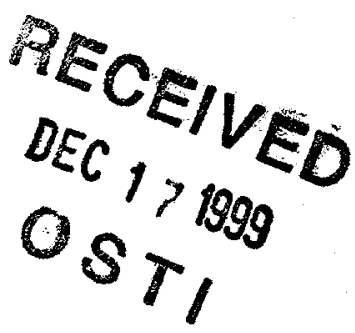

WSMS

O. Rivera

WSMS

DOE Contract No. DE-AC09-96SR18500

This paper was prepared in connection with work done under the above contract number with the $U$. S. Department of Energy. By acceptance of this paper, the publisher and/or recipient acknowledges the U. S. Government's right to retain a nonexclusive, royalty-free license in and to any copyright covering this paper, along with the right to reproduce and to authorize others to reproduce all or part of the copyrighted paper. 


\section{DISCLAIMER}

This report was prepared as an account of work sponsored by an agency of the United States Government. Neither the United States Government nor any agency thereof, nor any of their employees, makes any warranty, express or implied, or assumes any legal liability or responsibility for the accuracy, completeness, or usefulness of any information, apparatus, product, or process disclosed, or represents that its use would not infringe privately owned rights. Reference herein to any specific commercial product, process, or service by trade name, trademark, manufacturer, or otherwise does not necessarily constitute or imply its endorsement, recommendation, or favoring by the United States Government or any agency thereof. The views and opinions of authors expressed herein do not necessarily state or reflect those of the United States Government or any agency thereof.

This report has been reproduced directly from the best available copy.

Available to DOE and DOE contractors from the Office of Scientific and Technical Information, P. O. Box 62, Oak Ridge, TN 37831; prices available from (423) 576-8401.

Available to the public from the National Technical Information Service, U. S. Department of Commerce, 5285 Port Royal Road, Springfield, VA 22161. 


\section{DISCLAIMER}

Portions of this document may be illegible in electronic image products. Images are produced from the best available original document. 
Westinghouse Safety Management Solutions LLC

Criticality and Radiation Transport Services

Criticality Technology Group

September 30, 1999

WSRC-TR-99-00349

KEYWORDS

BUGLE-96

MORSE-SGC/S

SCALE 4.3

UNCLASSIFIED

SINBAD 97

Does Not Contain

Unclassified Controlled Nuclear Information

ADC \& Reviewing

Official:

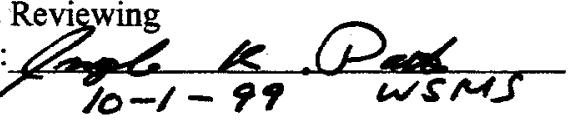

RETENTION:

Lifetime

BUGLE-96 VALIDATION WITH MORSE-SGC/S

USING WATER AND IRON EXPERIMENTS FROM SINBAD 97

APPROVALS:

Author:

Criticality Technology

Author:

Criticality Technology

Technical Reviewer:

Radiological \& Spent Fuel Engineering

Manager:

Criticality technology
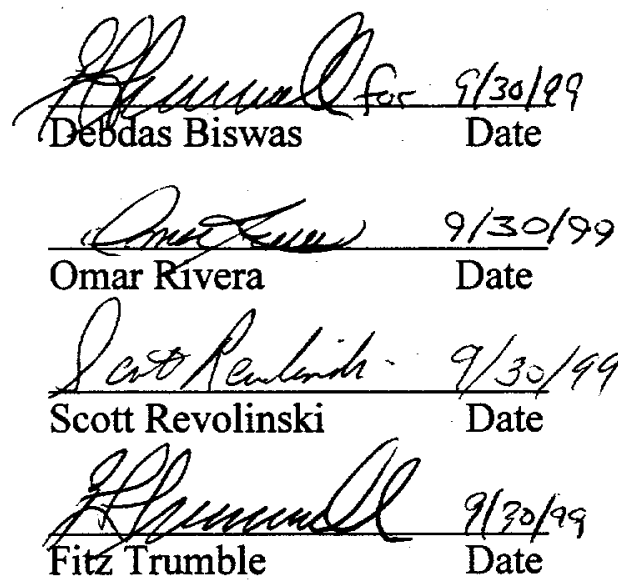

\section{DISTRIBUTION:}

J. Brotherton, CCC-3 E.F. Trumble, CCC-3

C.E. Apperson, CCC-3

B.T. Baranko, CCC-3

D. Biswas, CCC-3

M.A. Rosser, 703-F
S. Nathan, CCC-3

O. Rivera, CCC-3

M. Harris, 707-C
S. Revolinski, CCC-3
T. White, $\mathrm{CCC}-3$

J. Thompson, $\mathrm{CCC}-3$

J. Hack, 730-2B

J. Bryce, 707-C

M. Nadeau, 707-C
N.J. Jordan, 707-C

WSMS/CRT Files

Records 773-51A

A. Blanchard, 730-B 


\section{TABLE OF CONTENTS}

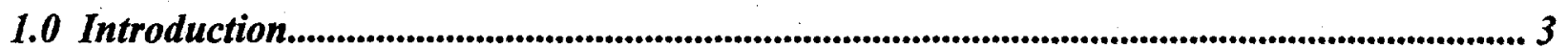

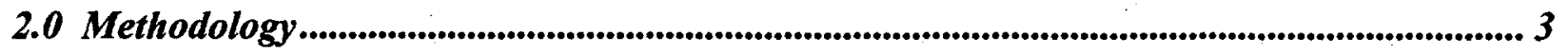

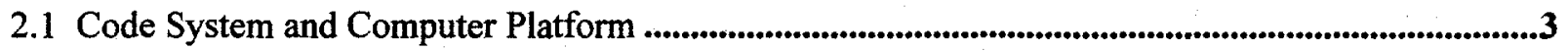

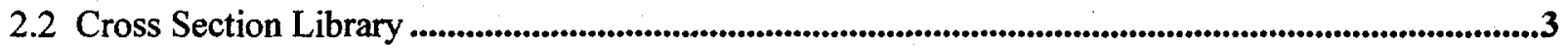

2.3 SINBAD 97 Shielding Database

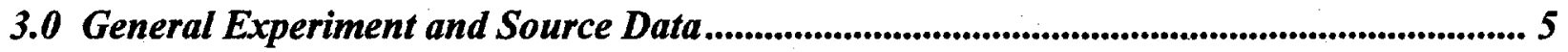

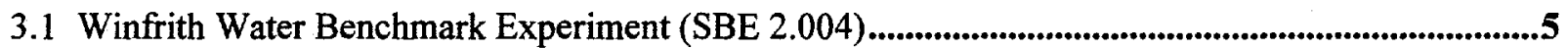

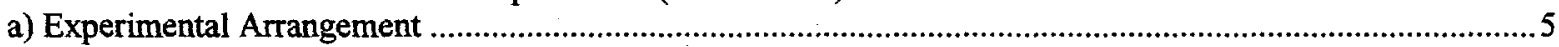

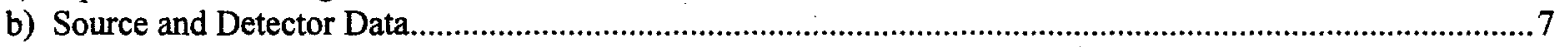

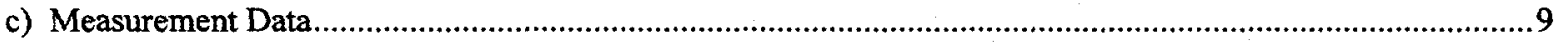

3.2 Karlsruhe Iron Sphere Benchmark Experiment (SBE 6.001) ........................................................17

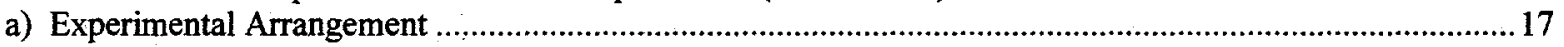

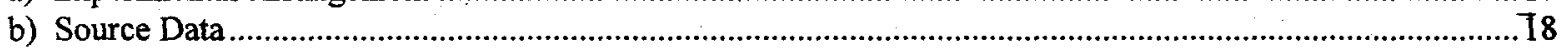

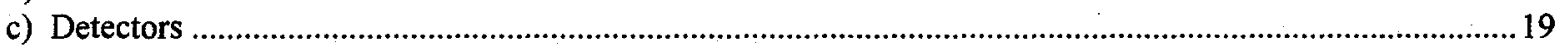

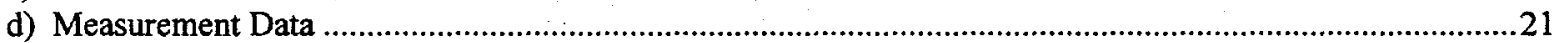

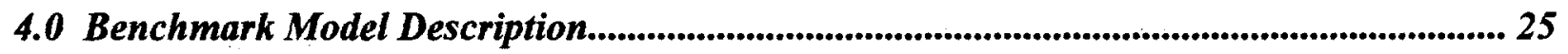

4.1 Winfrith Water Benchmark Model ............................................................................................................25

4.2 Karlsruhe Iron Sphere Benchmark Model............................................................................................26

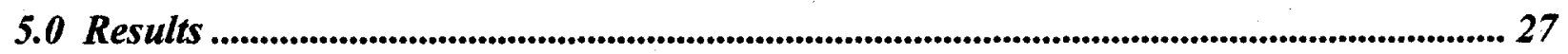

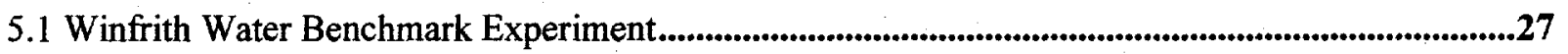

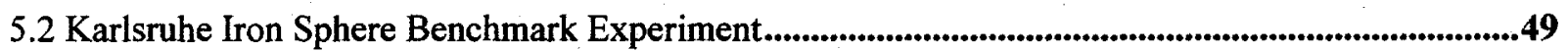

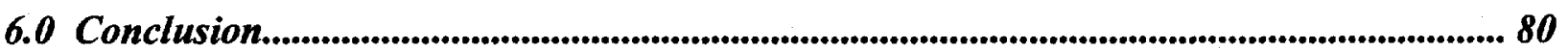

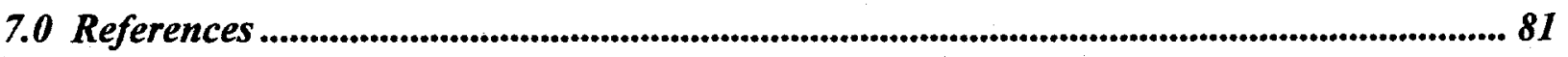

APPENDIX A: Input Listings for Winfrith Water Benchmark Experiment ........................... 82

APPENDIX B: Input Listings for Karlsruhe Iron Sphere Benchmark Experiment................ 86 
BUGLE 96 VALIDATION WITH MORSE USING WATER

WSRC-TR-00349

AND IRON EXPERIMENTAL DATA FROM SINDBAD 97

Rev.0

Page 3 of 89

\subsection{Introduction}

This document summarizes the validation of MORSE-SGC/S [1] with the BUGLE-96 cross section library [2]. SINBAD [3] Benchmark Experiment (SBE) 2.004, Winfrith Water Benchmark Experiment and SBE 6.001, Karlsruhe Iron Sphere Benchmark Experiment were utilized for this validation. The MORSE-SGC/S code with the BUGLE-96 cross-section library was used to model the experimental configurations as given in SINDBAD 97. SINDBAD is a shielding integral benchmark archive and database developed at the Oak Ridge National Laboratory (ORNL). For means of comparison, the experimental models were also executed with MORSE-SGC/S using the BUGLE-80 cross-section library. BUGLE-96 cross section will be used for shielding applications only as recommended by ORNL.

\subsection{Methodology}

\subsection{Code System and Computer Platform}

MORSE-SGC/S is the SCALE version of the MORSE-SGC code, a member of the MORSE Monte Carlo family of codes developed at Oak Ridge National Laboratory (ORNL). MORSESGC is unique from other versions of MORSE since it contains supergroup cross-section storage and tracking features along with an improved geometry system (MARS, Multiple ARray System) for nested rectangular array analysis. The basic theory behind all the MORSE codes is identical, however, and has been documented in Ref. 1 .

The MORSE-SGC/S modular code system was received from the Radiation Shielding Information Center (RSIC) as part of the SCALE 4:3 package [4]. It was installed on the WSMS Digital Equipment Alpha Processors (DEC Alpha) UNIX Workstation cluster, where the validation calculations were executed [5].

\subsection{Cross Section Library}

A new multigroup cross-section library based on ENDF/B-VI data was used. This broad-group (47 neutron, 20 gamma-ray) library, designated as DLC-185/ BUGLE-96 (or simply BUGLE96), is intended to replace the DLC-75/BUGLE-80 and DLC-76/SAILOR libraries, which are both based on ENDF/B-IV data. It also replaces DLC-175/BUGLE-93 library by correcting some deficiencies and adding several additional data sets.

The BUGLE-96 cross section library was produced and tested for light water reactor shielding and reactor pressure vessel dosimetry applications. It contains 120 nuclides, which have been processed as infinitely dilute and collapsed using an LWR concrete shield spectrum. Additionally, it contains 105 nuclides which have been energy self-shielded and collapsed using LWR-specific material composition and flux spectra. 
BUGLE 96 VALIDATION WITH MORSE USING WATER

WSRC-TR-00349

AND IRON EXPERIMENTAL DATA FROM SINDBAD 97

Rev.0

Page 4 of 89

BUGLE-96 cross section set was obtained from RSICC. The BUGLE 96 library needs to be converted from the ANISN format to an AMPX working library format before it can be used with the functional modules in SCALE like MORSE or XSDRNPM. This is different from the 27-group or the 238-group library in master library format commonly used in SCALE.

If one wants to run with the SCALE control modules like SAS1, SAS3 or SAS4, VITAMIN-B library in master library format may be used. This is the 199-group library from which BUGLE96 was collapsed.

There are two BUGLE files in the DLC-185 set. One is BUGLE-96 and the other is BUGLE96T. In our study, BUGLE-96T is used, because it is the same cross section set as BUGLE-96 with the thermal upscattering cross sections retained.

We have used the SCALE module LAVA to convert BUGLE-96T from ANISN to AMPX format. A formatting problem was found in the original BUGLE-96T file. The problem has been corrected and the corresponding modified file is called BUGLE-96T1. The normal scale scripts (subscale, smr_scale4.3_script, batch43 (here called lava43) and scale43 (here called scale96t) have been used to run LAVA with an input deck called lava96t.in to produce an ampx formatted library called bugle96_ampx.bin.

\subsection{SINBAD 97 Shielding Database}

SINBAD 97/DLC-191, a shielding integral benchmark archive and database was developed at ORNL and was obtained from RSICC. SINBAD is an active and growing radiation benchmark catalogue that provides the most accurate and complete electronic data available from bulk shielding experiments. SINBAD 97 contains ten (10) fission and one (1) fusion benchmark compilations for shielding analysis. The Winfrith (ASPIS) water benchmark experiment and the Karlsruhe iron benchmark experiment were selected for MORSE- SGC/S validation with BUGLE-96 for this initial phase and more experiments will be used in the future subject to the availability of funding. The details of the experiments are outlined in Section 3. 
BUGLE 96 VALIDATION WITH MORSE USING WATER

WSRC-TR-00349

AND IRON EXPERIMENTAL DATA FROM SINDBAD 97

Rev.0

Page 5 of 89

\subsection{General Experiment and Source Data}

The general experimental arrangements, detector locations, and source term data for the Winfrith water experiments and the Karlsruhe iron experiments are given in Sections 3.1 and 3.2, respectively.

\subsection{Winfrith Water Benchmark Experiment (SBE 2.004)}

Seven sets of experiments were analyzed with different source to detector distance. Cf-252 sources were used in the experiments. The 47 -group Cf- 252 spontaneous fission spectrum data (identical to Bugle-96 group structure) was obtained from the University of Illinois Iron Benchmark evaluation (1975) as documented in SINBAD 97.

a) Experimental Arrangement

The general arrangement of the experiment (shown in Figure 3.1-1) consists of a light structure supporting a vertical aluminium tube in which the measurements are made. Eight neutron sources are suspended from the arms of the support structure at predetermined and accurately known distances from the axis of the measurement tube. The sources are suspended at equal distances from the arms. All measurements are made in the source plane and at distances of 15 $\mathrm{cm}$ and $30 \mathrm{~cm}$ above and below the source plane in the air-filled measurement tube. The support structure is located in a water-filled aluminium tank with a cross-section of $228 \mathrm{~cm} \times 177 \mathrm{~cm}$ and a height of $172 \mathrm{~cm}$. The nearest distance of a source to any external boundary during any measurement was approximately $38 \mathrm{~cm}$. Since this is more than four times the migration length of $5 \mathrm{MeV}$ neutrons in water, the whole source-detector arrangement can be safely treated as being immersed in an infinite bath of water. 


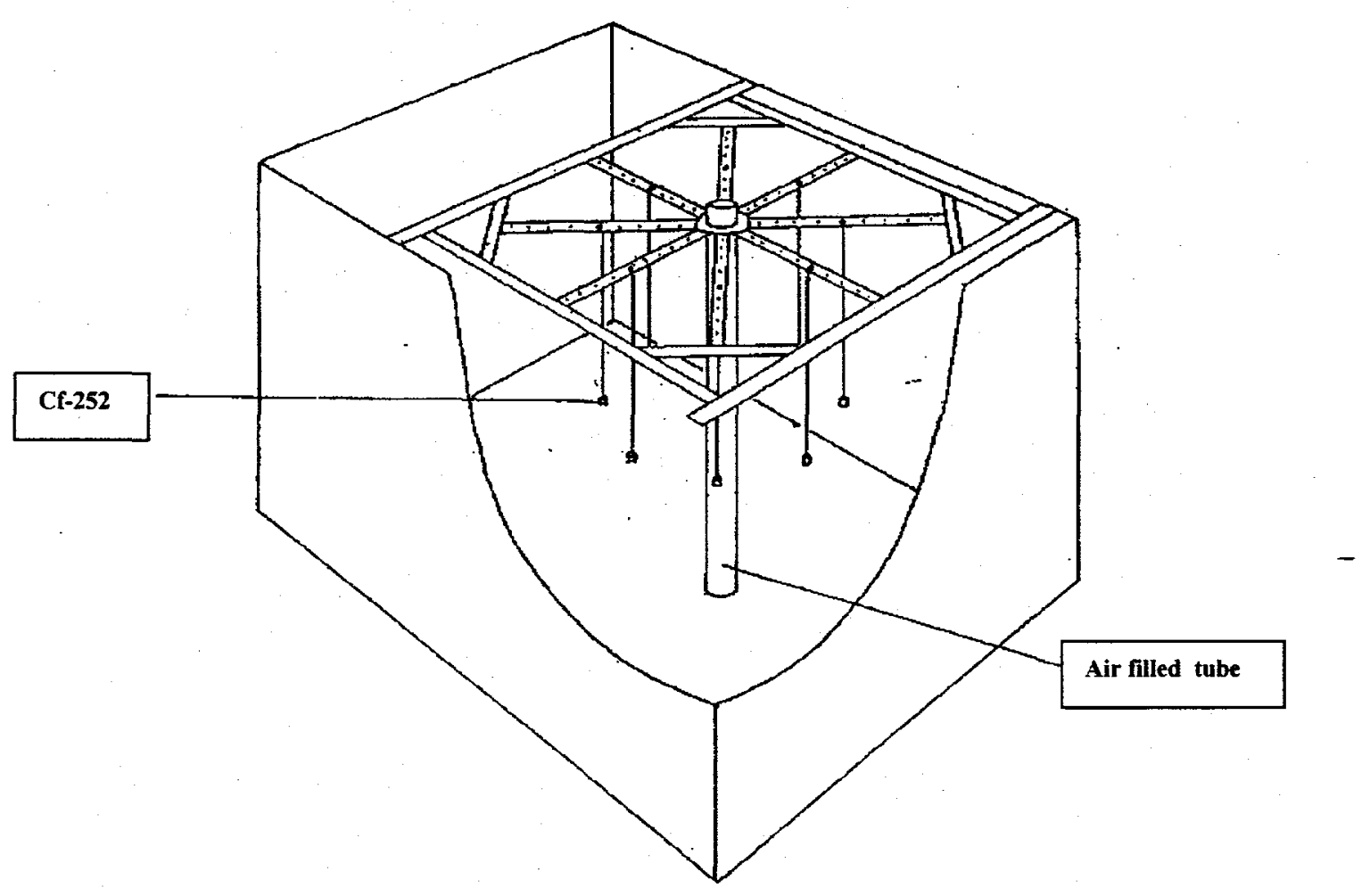

Figure 3.1-1: Schematic of the benchmark experiment.

Figure 3.1-2 shows in detail the source-detector arrangement with one source at the minimum separation distance of $101.6 \mathrm{~mm}$ from the center of the detector. This separation distance can be increased in units of $50.8 \mathrm{~mm}$ and up to eight sources can be positioned symmetrically about the detector. The inner source capsule is contained by double stainless steel walls of total thickness $1.6 \mathrm{~mm}$ and inserted via a plug (not shown in the Figure) into an outer cylindrical stainless steel container.

The neck of the outer container contains a steel screw, which is connected by very fine steel wire to an arm of the support structure. The central region of the source capsule contains a very small amount of air and aluminium. An exact geometric model of the source is given in Figure 3.1-3. 


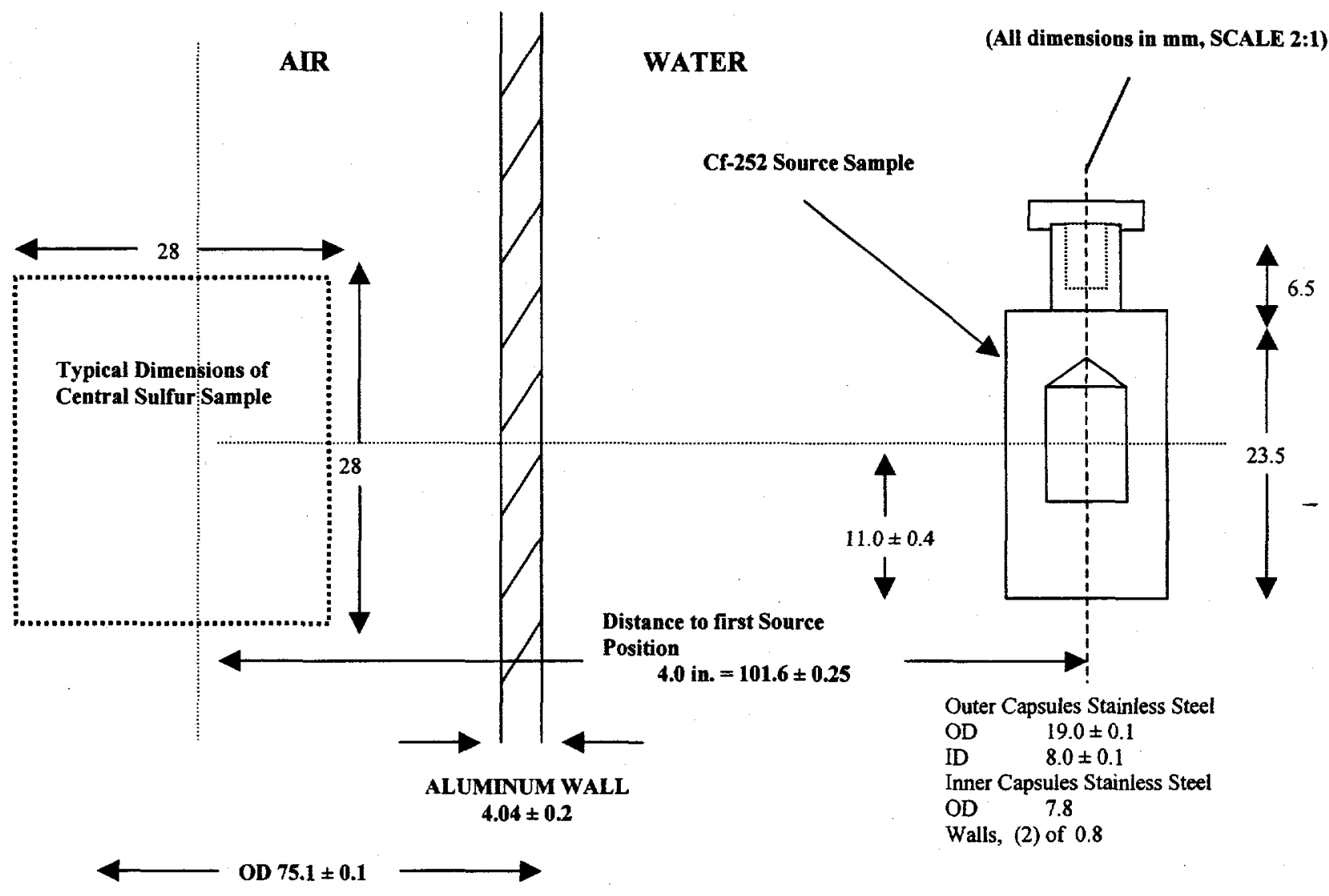

Figure 3.1-2: Detail of the Water Benchmark Experiment

b) Source and Detector Data

The neutron source in this experiment was provided by spontaneous fission from Cf-252. Eight individual sources, calibrated at the National Physical Laboratory, were available with a total output of $1.048 \mathrm{E}+8$ neutrons/second. The composition of the source is given in Table 1 .

Absolute calibration of the sources was carried out at the National Physical Laboratory, Teddington, in April 1981 using manganese bath method. The estimated standard deviation on the source strengths is $0.5 \%$ and in the measured results the appropriate (and very small) corrections for half-life have been made.

The sulfur detectors were cast into cylindrical blocks of the same nominal size but whose dimensions vary slightly. The nominal size sample is shown in Figure 3.1-2. 


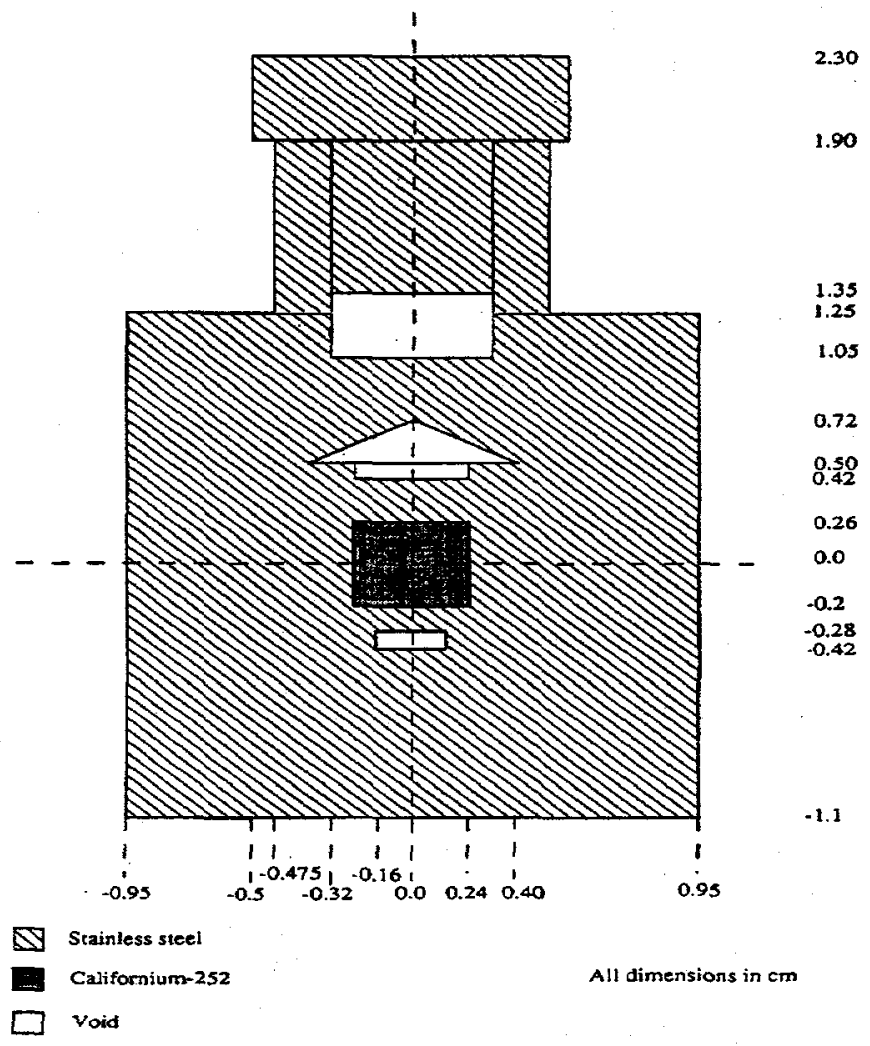

Figure 3.1-3: Model of the source capsule.

Table 3.1-1: Composition of the Source Capsule (see Figure 3 for region definitions).

\begin{tabular}{|c|c|c|c|c|}
\hline Region & Material & Density $\left(\mathrm{g} / \mathrm{cm}^{3}\right)$ & Composition & Proportion by weight \\
\hline 1 & $\mathrm{U}^{*} 238^{*}$ & 18.9 & $\mathrm{U}-238$ & 1.0 \\
\hline 2 & Stainless Steel & 7.9 & $\mathrm{Mn}$ & 0.01587 \\
& & & $\mathrm{Cr}$ & 0.1868 \\
& & & $\mathrm{Ni}$ & 0.09504 \\
& & & $\mathrm{Fe}$ & 0.703 \\
\hline 3 & Air Gaps & & $\mathrm{Void}$ & - \\
\hline 4 & Aluminium & 2.7 & $\mathrm{Al}$ & 1.0 \\
\hline 5 & Water & 1.0 & $\mathrm{H}$ & 0.1119 \\
& & & $\mathrm{O}$ & 0.8881 \\
\hline 6 & Sulfur & 1.86 & $\mathrm{~S}$ & 1.0 \\
\hline
\end{tabular}

Cf-252 actually used in the experiment is replaced by U-238 in the calculations. 
BUGLE 96 VALIDATION WITH MORSE USING WATER

WSRC-TR-00349

AND IRON EXPERIMENTAL DATA FROM SINDBAD 97

Rev. 0

Page 9 of 89

c) Measurement Data

The information provided in Tables 3.1-3 to 3.1-9 was taken from Reference 3 as unfolded by RADAK [6]. The tables show the spectra determined by a $3 \mathrm{ml} \mathrm{NE}-213$ organic liquid scintillator at seven different source-detector separations. The fractional standard deviations shown in column 5 derive from random counting statistics only; in the last column, to these was added quadratically a contribution of $5 \%$ attributable to uncertainty in the volume of the detector. This contribution is systematic throughout the measurements. The results in tables 3.1-3 to 3.1-9 are appropriate to the total source strength given in Table 3.1-2.

Reference 3 also provided the S-32(n,p)P-32 saturated reaction rates per sulfur atom per source neutron for the various source-detector separations. These are listed in Table 3.1-10. The 6\% standard deviation shown for these measurements is caused by dispersion arising from the experimental method rather than from counting statistics. (The experimental values given for the off axis positions are the mean of the above and below axis measurements.) The cast sulfur samples had a density of $1.86+-5 \% \mathrm{~g} / \mathrm{cm}^{3}$.

Table 3.1-2: Experimental Source Arrangements

\begin{tabular}{|c|c|c|c|}
\hline Case & $\begin{array}{c}\text { Source-detector Spacing } \\
(\mathrm{cm})\end{array}$ & $\begin{array}{c}\text { Number of } \\
\text { Sources }\end{array}$ & $\begin{array}{c}\text { Total Source Strength } \\
(\mathrm{n} / \mathrm{s})\end{array}$ \\
\hline 1 & 10.16 & 1 & $1.26 \mathrm{E}+07$ \\
\hline 2 & 15.24 & 2 & $2.54 \mathrm{E}+07$ \\
\hline 3 & 20.32 & 4 & $5.26 \mathrm{E}+07$ \\
\hline 4 & 25.40 & 8 & $1.05 \mathrm{E}+08$ \\
\hline 5 & 30.48 & 8 & $1.05 \mathrm{E}+08$ \\
\hline 6 & 35.56 & 8 & $1.05 \mathrm{E}+08$ \\
\hline 7 & 50.80 & 8 & $1.04 \mathrm{E}+08$ \\
\hline
\end{tabular}


BUGLE 96 VALIDATION WITH MORSE USING WATER

WSRC-TR-00349

AND IRON EXPERIMENTAL DATA FROM SINDBAD 97

Rev.0

Page 10 of 89

Table 3.1-3: Neutron Spectrum at $10.16 \mathrm{~cm}$

\begin{tabular}{|c|c|c|c|c|c|}
\hline Group & $\begin{array}{c}\text { Energy Boundaries } \\
(\mathrm{MeV})\end{array}$ & $\begin{array}{c}\text { Flux/Lethargy } \\
\left(\mathrm{cm}^{-2} / \mathrm{s}\right)\end{array}$ & $\begin{array}{c}\text { Flux/Lethargy per } \\
\text { Source Neutron } \\
\left(\mathrm{cm}^{-2} / \mathrm{s}\right)\end{array}$ & $\begin{array}{c}\text { Std. Deviation } \\
(\text { statistics only) } \\
(\%)\end{array}$ & $\begin{array}{c}\text { Std. Deviation } \\
\text { (total) } \\
(\%)\end{array}$ \\
\hline & 10.000 & & & & \\
19 & 8.825 & $8.50 \mathrm{E}+01$ & $6.77 \mathrm{E}-06$ & 13.6 & 14.5 \\
18 & 7.788 & $2.07 \mathrm{E}+02$ & $1.65 \mathrm{E}-05$ & 9.0 & 10.3 \\
17 & 6.873 & $3.45 \mathrm{E}+02$ & $2.75 \mathrm{E}-05$ & 6.3 & 8.0 \\
16 & 6.065 & $5.80 \mathrm{E}+02$ & $4.62 \mathrm{E}-05$ & 4.4 & 6.7 \\
15 & 5.353 & $8.36 \mathrm{E}+02$ & $6.66 \mathrm{E}-05$ & 3.0 & 5.8 \\
14 & 4.724 & $1.15 \mathrm{E}+03$ & $9.15 \mathrm{E}-05$ & 2.6 & 5.6 \\
13 & 4.169 & $1.33 \mathrm{E}+03$ & $1.06 \mathrm{E}-04$ & 2.0 & 5.4 \\
12 & 3.679 & $1.70 \mathrm{E}+03$ & $1.35 \mathrm{E}-04$ & 1.5 & 5.2 \\
11 & 3.247 & $1.82 \mathrm{E}+03$ & $1.45 \mathrm{E}-04$ & 1.7 & 5.3 \\
10 & 2.865 & $2.25 \mathrm{E}+03$ & $1.79 \mathrm{E}-04$ & 1.5 & 5.2 \\
9 & 2.528 & $2.61 \mathrm{E}+03$ & $2.08 \mathrm{E}-04$ & 1.3 & 5.2 \\
8 & 2.231 & $2.68 \mathrm{E}+03$ & $2.13 \mathrm{E}-04$ & 1.2 & 5.1 \\
7 & 1.969 & $2.64 \mathrm{E}+03$ & $2.10 \mathrm{E}-04$ & 2.0 & 5.4 \\
6 & 1.738 & $2.48 \mathrm{E}+03$ & $1.98 \mathrm{E}-04$ & 1.8 & 5.3 \\
5 & 1.534 & $2.49 \mathrm{E}+03$ & $1.98 \mathrm{E}-04$ & 2.0 & 5.4 \\
4 & 1.353 & $2.54 \mathrm{E}+03$ & $2.02 \mathrm{E}-04$ & 2.1 & 5.4 \\
3 & 1.194 & $2.26 \mathrm{E}+03$ & $1.80 \mathrm{E}-04$ & 3.3 & 6.0 \\
2 & 1.054 & $2.34 \mathrm{E}+03$ & $1.85 \mathrm{E}-04$ & 3.4 & 6.0 \\
1 & 0.930 & $2.02 \mathrm{E}+03$ & $1.60 \mathrm{E}-04$ & 2.4 & 5.5 \\
\hline
\end{tabular}


Table 3.1-4: Neutron Spectrum at $15.24 \mathrm{~cm}$

\begin{tabular}{|c|c|c|c|c|c|}
\hline Group & $\begin{array}{c}\text { Energy Boundaries } \\
(\mathrm{MeV})\end{array}$ & $\begin{array}{c}\text { Flux/Lethargy } \\
\left(\mathrm{cm}^{-2} / \mathrm{s}\right)\end{array}$ & $\begin{array}{c}\text { Flux/Lethargy per } \\
\text { Source Neutron }\left(\mathrm{cm}^{-}\right. \\
\text {/s) }\end{array}$ & $\begin{array}{c}\text { Std. Deviation } \\
(\text { statistics only }) \\
(\%)\end{array}$ & $\begin{array}{c}\text { Std. Deviation } \\
\text { (total) } \\
(\%)\end{array}$ \\
\hline & 10.000 & & & & \\
19 & 8.825 & $6.78 \mathrm{E}+01$ & $2.67 \mathrm{E}-06$ & 9.2 & 10.5 \\
18 & 7.788 & $1.12 \mathrm{E}+02$ & $4.40 \mathrm{E}-06$ & 7.1 & 8.7 \\
17 & 6.873 & $2.12 \mathrm{E}+02$ & $8.33 \mathrm{E}-06$ & 3.7 & 6.2 \\
16 & 6.065 & $3.59 \mathrm{E}+02$ & $1.41 \mathrm{E}-04$ & 3.0 & 5.8 \\
15 & 5.353 & $4.57 \mathrm{E}+02$ & $1.80 \mathrm{E}-05$ & 2.9 & 5.8 \\
14 & 4.724 & $5.98 \mathrm{E}+02$ & $2.35 \mathrm{E}-05$ & 2.0 & 5.4 \\
13 & 4.169 & $7.77 \mathrm{E}+02$ & $3.06 \mathrm{E}-05$ & 1.4 & 5.2 \\
12 & 3.679 & $7.84 \mathrm{E}+02$ & $3.09 \mathrm{E}-05$ & 1.8 & 5.3 \\
11 & 3.247 & $8.64 \mathrm{E}+02$ & $3.40 \mathrm{E}-05$ & 1.7 & 5.3 \\
10 & 2.865 & $1.10 \mathrm{E}+03$ & $4.32 \mathrm{E}-05$ & 1.2 & 5.1 \\
9 & 2.528 & $1.23 \mathrm{E}+03$ & $4.83 \mathrm{E}-05$ & 1.2 & 5.1 \\
8 & 2.231 & $1.38 \mathrm{E}+03$ & $5.41 \mathrm{E}-05$ & 1.5 & 5.2 \\
7 & 1.969 & $1.21 \mathrm{E}+03$ & $4.76 \mathrm{E}-05$ & 1.9 & 5.3 \\
6 & 1.738 & $1.13 \mathrm{E}+03$ & $4.45 \mathrm{E}-05$ & 1.5 & 5.2 \\
5 & 1.534 & $1.18 \mathrm{E}+03$ & $4.63 \mathrm{E}-05$ & 0.8 & 5.1 \\
4 & 1.353 & $1.15 \mathrm{E}+03$ & $4.52 \mathrm{E}-05$ & 1.2 & 5.1 \\
3 & 1.194 & $9.83 \mathrm{E}+02$ & $3.87 \mathrm{E}-05$ & 1.7 & 5.3 \\
2 & 1.054 & $1.09 \mathrm{E}+03$ & $4.27 \mathrm{E}-05$ & 1.3 & 5.2 \\
1 & 0.930 & $8.86 \mathrm{E}+02$ & $3.49 \mathrm{E}-05$ & 1.2 & 5.1 \\
\hline
\end{tabular}


BUGLE 96 VALIDATION WITH MORSE USING WATER AND IRON EXPERIMENTAL DATA FROM SINDBAD 97
WSRC-TR-00349

Rev.0

Page 12 of 89

Table 3.1-5: Neutron Spectrum at $20.32 \mathrm{~cm}$

\begin{tabular}{|c|c|c|c|c|c|}
\hline Group & $\begin{array}{c}\text { Energy Boundaries } \\
(\mathrm{MeV})\end{array}$ & $\begin{array}{c}\text { Flux/Lethargy } \\
\left(\mathrm{cm}^{-2} / \mathrm{s}\right)\end{array}$ & $\begin{array}{c}\text { Flux/Lethargy per } \\
\text { Source Neutron }\left(\mathrm{cm}^{-}\right. \\
2 / \mathrm{s})\end{array}$ & $\begin{array}{c}\text { Std. Deviation } \\
(\text { statistics only) } \\
(\%)\end{array}$ & $\begin{array}{c}\text { Std. Deviation } \\
\text { (total) } \\
(\%)\end{array}$ \\
\hline 19 & 10.000 & & & & 7.0 \\
18 & 8.825 & $5.49 \mathrm{E}+01$ & $1.04 \mathrm{E}-06$ & 6.0 & 7.6 \\
17 & 7.788 & $8.53 \mathrm{E}+01$ & $1.62 \mathrm{E}-06$ & 3.8 & 6.3 \\
16 & 6.873 & $1.56 \mathrm{E}+02$ & $2.96 \mathrm{E}-06$ & 2.7 & 5.7 \\
15 & 6.065 & $2.49 \mathrm{E}+02$ & $4.74 \mathrm{E}-06$ & 2.9 & 5.8 \\
14 & 5.353 & $3.19 \mathrm{E}+02$ & $6.07 \mathrm{E}-06$ & 2.0 & 5.4 \\
13 & 4.724 & $4.39 \mathrm{E}+02$ & $8.35 \mathrm{E}-06$ & 1.9 & 5.3 \\
12 & 4.169 & $4.81 \mathrm{E}+02$ & $9.14 \mathrm{E}-06$ & 1.7 & 5.3 \\
11 & 3.679 & $5.05 \mathrm{E}+02$ & $9.60 \mathrm{E}-06$ & 1.4 & 5.3 \\
10 & 3.247 & $5.11 \mathrm{E}+02$ & $9.72 \mathrm{E}-06$ & 1.1 & 5.2 \\
9 & 2.865 & $6.80 \mathrm{E}+02$ & $1.29 \mathrm{E}-05$ & 0.7 & 5.1 \\
8 & 2.528 & $7.54 \mathrm{E}+02$ & $1.43 \mathrm{E}-05$ & 0.9 & 5.1 \\
7 & 2.231 & $8.56 \mathrm{E}+02$ & $1.63 \mathrm{E}-05$ & 1.3 & 5.2 \\
6 & 1.969 & $7.45 \mathrm{E}+02$ & $1.42 \mathrm{E}-05$ & 1.4 & 5.2 \\
5 & 1.738 & $7.08 \mathrm{E}+02$ & $1.35 \mathrm{E}-05$ & 1.1 & 5.1 \\
4 & 1.534 & $6.70 \mathrm{E}+02$ & $1.28 \mathrm{E}-05$ & 1.4 & 5.2 \\
3 & 1.353 & $7.03 \mathrm{E}+02$ & $1.34 \mathrm{E}-05$ & 1.5 & 5.2 \\
2 & 1.194 & $6.03 \mathrm{E}+02$ & $1.15 \mathrm{E}-05$ & 1.3 & 5.2 \\
1 & 1.054 & $6.85 \mathrm{E}+02$ & $1.30 \mathrm{E}-05$ & & \\
\hline
\end{tabular}


BUGLE 96 VALIDATION WITH MORSE USING WATER

WSRC-TR-00349

AND IRON EXPERIMENTAL DATA FROM SINDBAD 97

Rev.0

Page 13 of 89

Table 3.1-6: Neutron Spectrum at $25.40 \mathrm{~cm}$

\begin{tabular}{|c|c|c|c|c|c|}
\hline Group & $\begin{array}{c}\text { Energy Boundaries } \\
(\mathrm{MeV})\end{array}$ & $\begin{array}{c}\text { Flux/Lethargy } \\
\left(\mathrm{cm}^{-2} / \mathrm{s}\right)\end{array}$ & $\begin{array}{c}\text { Flux/Lethargy per } \\
\text { Source Neutron } \\
\left(\mathrm{cm}^{-2} / \mathrm{s}\right)\end{array}$ & $\begin{array}{c}\text { Std. Deviation } \\
(\text { statistics only }) \\
(\%)\end{array}$ & $\begin{array}{c}\text { Std. Deviation } \\
\text { (total) } \\
(\%)\end{array}$ \\
\hline & 10.000 & & & & \\
19 & 8.825 & $3.92 \mathrm{E}+01$ & $3.74 \mathrm{E}-07$ & 9.0 & 10.3 \\
18 & 7.788 & $7.50 \mathrm{E}+01$ & $7.15 \mathrm{E}-07$ & 6.0 & 7.8 \\
17 & 6.873 & $1.20 \mathrm{E}+02$ & $1.14 \mathrm{E}-06$ & 4.2 & 6.5 \\
16 & 6.065 & $1.92 \mathrm{E}+02$ & $1.83 \mathrm{E}-06$ & 3.2 & 5.9 \\
15 & 5.353 & $2.55 \mathrm{E}+02$ & $2.44 \mathrm{E}-06$ & 3.3 & 6.0 \\
14 & 4.724 & $3.18 \mathrm{E}+02$ & $3.03 \mathrm{E}-06$ & 2.7 & 5.7 \\
13 & 4.169 & $3.54 \mathrm{E}+02$ & $3.38 \mathrm{E}-06$ & 2.0 & 5.4 \\
12 & 3.679 & $3.62 \mathrm{E}+02$ & $3.46 \mathrm{E}-06$ & 1.8 & 5.3 \\
11 & 3.247 & $3.43 \mathrm{E}+02$ & $3.27 \mathrm{E}-06$ & 2.0 & 5.4 \\
10 & 2.865 & $4.44 \mathrm{E}+02$ & $4.24 \mathrm{E}-06$ & 1.7 & 5.3 \\
9 & 2.528 & $5.30 \mathrm{E}+02$ & $5.06 \mathrm{E}-06$ & 1.5 & 5.2 \\
8 & 2.231 & $5.80 \mathrm{E}+02$ & $5.53 \mathrm{E}-06$ & 1.4 & 5.2 \\
7 & 1.969 & $5.20 \mathrm{E}+02$ & $4.96 \mathrm{E}-06$ & 1.4 & 5.2 \\
6 & 1.738 & $4.73 \mathrm{E}+02$ & $4.51 \mathrm{E}-06$ & 1.6 & 5.2 \\
5 & 1.534 & $4.75 \mathrm{E}+02$ & $4.53 \mathrm{E}-06$ & 1.3 & 5.2 \\
4 & 1.353 & $4.77 \mathrm{E}+02$ & $4.55 \mathrm{E}-06$ & 1.6 & 5.2 \\
3 & 1.194 & $4.40 \mathrm{E}+02$ & $4.19 \mathrm{E}-06$ & 2.7 & 5.7 \\
2 & 1.054 & $5.21 \mathrm{E}+02$ & $4.97 \mathrm{E}-06$ & 2.3 & 5.5 \\
1 & 0.93 & $4.26 \mathrm{E}+02$ & $4.07 \mathrm{E}-06$ & 4.0 & 6.4 \\
\hline
\end{tabular}


BUGLE 96 VALIDATION WITH MORSE USING WATER AND IRON EXPERIMENTAL DATA FROM SINDBAD 97
WSRC-TR-00349

Rev.0

Page 14 of 89

Table 3.1-7: Neutron Spectrum at $30.48 \mathrm{~cm}$

\begin{tabular}{|c|c|c|c|c|c|}
\hline Group & $\begin{array}{c}\text { Energy Boundaries } \\
(\mathrm{MeV})\end{array}$ & $\begin{array}{c}\text { Flux/Lethargy } \\
\left(\mathrm{cm}^{-2} / \mathrm{s}\right)\end{array}$ & $\begin{array}{c}\text { Flux/Lethargy per } \\
\text { Source Neutron } \\
\left(\mathrm{cm}^{-2} / \mathrm{s}\right)\end{array}$ & $\begin{array}{c}\text { Std. Deviation } \\
\text { (statistics only) } \\
(\%)\end{array}$ & $\begin{array}{c}\text { Std. Deviation } \\
\text { (total) } \\
(\%)\end{array}$ \\
\hline 19 & 10.000 & & & & \\
18 & 8.825 & $1.85 \mathrm{E}+01$ & $1.77 \mathrm{E}-07$ & 7.4 & 8.9 \\
17 & 7.788 & $3.19 \mathrm{E}+01$ & $3.05 \mathrm{E}-07$ & 4.5 & 6.7 \\
16 & 6.873 & $5.71 \mathrm{E}+01$ & $5.46 \mathrm{E}-07$ & 2.6 & 5.6 \\
15 & 6.065 & $8.41 \mathrm{E}+01$ & $8.04 \mathrm{E}-07$ & 2.6 & 5.6 \\
14 & 5.353 & $1.02 \mathrm{E}+02$ & $9.77 \mathrm{E}-07$ & 2.2 & 5.5 \\
13 & 4.724 & $1.29 \mathrm{E}+02$ & $1.24 \mathrm{E}-06$ & 1.6 & 5.2 \\
12 & 4.169 & $1.42 \mathrm{E}+02$ & $1.36 \mathrm{E}-06$ & 1.4 & 5.2 \\
11 & 3.679 & $1.32 \mathrm{E}+02$ & $1.26 \mathrm{E}-06$ & 1.4 & 5.2 \\
10 & 3.247 & $1.26 \mathrm{E}+02$ & $1.20 \mathrm{E}-06$ & 1.0 & 5.2 \\
9 & 2.865 & $1.65 \mathrm{E}+02$ & $1.58 \mathrm{E}-06$ & 0.8 & 5.1 \\
8 & 2.528 & $1.89 \mathrm{E}+02$ & $1.81 \mathrm{E}-06$ & 0.9 & 5.1 \\
7 & 2.231 & $2.13 \mathrm{E}+02$ & $2.03 \mathrm{E}-06$ & 1.5 & 5.1 \\
6 & 1.969 & $1.83 \mathrm{E}+02$ & $1.75 \mathrm{E}-06$ & 1.6 & 5.2 \\
5 & 1.738 & $1.69 \mathrm{E}+02$ & $1.62 \mathrm{E}-06$ & 1.4 & 5.2 \\
4 & 1.534 & $1.64 \mathrm{E}+02$ & $1.57 \mathrm{E}-06$ & 1.2 & 5.2 \\
3 & 1.353 & $1.66 \mathrm{E}+02$ & $1.59 \mathrm{E}-06$ & 1.8 & 5.1 \\
2 & 1.194 & $1.52 \mathrm{E}+02$ & $1.45 \mathrm{E}-06$ & 1.5 & 5.3 \\
1 & 1.054 & $1.82 \mathrm{E}+02$ & $1.75 \mathrm{E}-06$ & 0.6 & 5.2 \\
& 0.93 & $1.69 \mathrm{E}+02$ & $1.61 \mathrm{E}-06$ & & 5.0 \\
\hline
\end{tabular}


BUGLE 96 VALIDATION WITH MORSE USING WATER

WSRC-TR-00349

AND IRON EXPERIMENTAL DATA FROM SINDBAD 97

Rev.0

Page 15 of 89

Table 3.1-8: Neutron Spectrum at $35.56 \mathrm{~cm}$

\begin{tabular}{|c|c|c|c|c|c|}
\hline Group & $\begin{array}{c}\text { Energy Boundaries } \\
(\mathrm{MeV})\end{array}$ & $\begin{array}{l}\text { Flux/Lethargy } \\
\left(\mathrm{cm}^{-2} / \mathrm{s}\right)\end{array}$ & $\begin{array}{c}\text { Flux/Lethargy per } \\
\text { Source Neutron } \\
\left(\mathrm{cm}^{-2} / \mathrm{s}\right)\end{array}$ & $\begin{array}{c}\text { Std. Deviation } \\
\text { (statistics only) } \\
(\%)\end{array}$ & $\begin{array}{l}\text { Std. Deviation } \\
\text { (total) } \\
(\%)\end{array}$ \\
\hline & 10.000 & & & & \\
\hline 19 & 8.825 & 9.62 & $9.17 \mathrm{E}-08$ & 9.0 & 10.3 \\
\hline 18 & 7.788 & $1.45 \mathrm{E}+01$ & $1.38 \mathrm{E}-07$ & 7.4 & 8.9 \\
\hline 17 & 6.873 & $2.47 \mathrm{E}+01$ & $2.36 \mathrm{E}-07$ & 4.8 & 6.9 \\
\hline 16 & 6.065 & $3.59 \mathrm{E}+01$ & $3.42 \mathrm{E}-07$ & 4.5 & 6.7 \\
\hline 15 & 5.353 & $4.25 \mathrm{E}+01$ & $4.06 \mathrm{E}-07$ & 3.9 & 6.3 \\
\hline 14 & 4.724 & $5.60 \mathrm{E}+01$ & $5.34 \mathrm{E}-07$ & 2.5 & 5.6 \\
\hline 13 & 4.169 & $5.67 \mathrm{E}+01$ & $5.41 \mathrm{E}-07$ & 2.2 & 5.5 \\
\hline 12 & 3.679 & $5.38 \mathrm{E}+01$ & $5.13 \mathrm{E}-07$ & 2.4 & 5.5 \\
\hline 11 & 3.247 & $4.46 \mathrm{E}+01$ & $4.26 \mathrm{E}-07$ & 3.8 & 6.3 \\
\hline 10 & 2.865 & $6.38 \mathrm{E}+01$ & $6.08 \mathrm{E}-07$ & 2.7 & 5.7 \\
\hline 9 & 2.528 & $6.92 \mathrm{E}+01$ & $6.60 \mathrm{E}-07$ & 2.6 & 5.6 \\
\hline 8 & 2.231 & $7.95 \mathrm{E}+01$ & $7.58 \mathrm{E}-07$ & 2.4 & 5.5 \\
\hline 7 & 1.969 & $6.74 \mathrm{E}+01$ & $6.43 \mathrm{E}-07$ & 2.1 & 5.4 \\
\hline 6 & 1.738 & $6.25 E+01$ & $5.96 \mathrm{E}-07$ & 2.0 & 5.4 \\
\hline 5 & 1.534 & $6.00 \mathrm{E}+01$ & 5.72E-07 & 2.3 & 5.5 \\
\hline 4 & 1.353 & $5.99 E+01$ & $5.71 E-07$ & 2.1 & 5.4 \\
\hline 3 & 1.194 & $5.43 \mathrm{E}+01$ & $5.18 \mathrm{E}-07$ & 1.3 & 5.2 \\
\hline 2 & 1.054 & $6.49 E+01$ & 6.19E-07 & 0.7 & 5.0 \\
\hline 1 & 0.93 & $5.64 \mathrm{E}+01$ & $5.38 \mathrm{E}-07$ & 0.5 & 5.0 \\
\hline
\end{tabular}


Table 3.1-9: Neutron Spectrum at $50.80 \mathrm{~cm}$

\begin{tabular}{|c|c|c|c|c|c|}
\hline Group & $\begin{array}{c}\text { Energy Boundaries } \\
(\mathrm{MeV})\end{array}$ & $\begin{array}{c}\text { Flux/Lethargy } \\
\left(\mathrm{cm}^{-2} / \mathrm{s}\right)\end{array}$ & $\begin{array}{c}\text { Flux/Lethargy per } \\
\text { Source Neutron } \\
\left(\mathrm{cm}^{-2} / \mathrm{s}\right)\end{array}$ & $\begin{array}{c}\text { Std. Deviation } \\
(\text { statistics only) } \\
(\%)\end{array}$ & $\begin{array}{c}\text { Std. Deviation } \\
(\text { total }) \\
(\%)\end{array}$ \\
\hline & 10.000 & & & & \\
19 & 8.825 & 1.43 & $1.36 \mathrm{E}-08$ & 8.3 & 9.7 \\
18 & 7.788 & 1.85 & $1.77 \mathrm{E}-08$ & 10.2 & 11.4 \\
17 & 6.873 & 2.79 & $2.67 \mathrm{E}-08$ & 8.8 & 10.1 \\
16 & 6.065 & 4.07 & $3.90 \mathrm{E}-08$ & 6.4 & 8.1 \\
15 & 5.353 & 4.82 & $4.61 \mathrm{E}-08$ & 6.1 & 7.9 \\
14 & 4.724 & 4.71 & $4.51 \mathrm{E}-08$ & 6.4 & 8.1 \\
13 & 4.169 & 5.49 & $5.26 \mathrm{E}-08$ & 4.7 & 6.9 \\
12 & 3.679 & 3.83 & $3.67 \mathrm{E}-08$ & 5.4 & 7.4 \\
11 & 3.247 & 4.21 & $4.03 \mathrm{E}-08$ & 3.9 & 6.3 \\
10 & 2.865 & 4.59 & $4.39 \mathrm{E}-08$ & 3.1 & 5.9 \\
9 & 2.528 & 5.08 & $4.87 \mathrm{E}-08$ & 3.5 & 6.1 \\
8 & 2.231 & 5.85 & $5.60 \mathrm{E}-08$ & 4.0 & 6.4 \\
7 & 1.969 & 4.58 & $4.39 \mathrm{E}-08$ & 5.7 & 7.6 \\
6 & 1.738 & 4.34 & $4.15 \mathrm{E}-08$ & 5.8 & 7.7 \\
5 & 1.534 & 4.32 & $4.14 \mathrm{E}-08$ & 4.1 & 6.5 \\
4 & 1.353 & 4.17 & $4.00 \mathrm{E}-08$ & 2.5 & 5.6 \\
3 & 1.194 & 3.94 & $3.77 \mathrm{E}-08$ & 1.7 & 5.3 \\
2 & 1.054 & 4.42 & $4.23 \mathrm{E}-08$ & 1.2 & 5.1 \\
1 & 0.93 & 4.61 & $4.41 \mathrm{E}-08$ & 3.4 & 6.0 \\
\hline
\end{tabular}

Table 3.1-10: $\mathrm{S}^{32}(\mathrm{n}, \mathrm{p}) \mathrm{P}^{32}$ Measured Reaction Rates

\begin{tabular}{|c|c|c|c|}
\hline \multirow{2}{*}{$\begin{array}{c}\text { Distance in Source } \\
\text { Plane of Sources from } \\
\text { Tube }(\mathrm{cm})\end{array}$} & \multicolumn{3}{|c|}{ Saturated Reaction Rates per $\mathrm{S}^{32}$ Atom per Source Neutron } \\
\cline { 2 - 4 } & 0 & $+/-15$ & $+/-30$ \\
\hline 10.26 & $3.13 \mathrm{E}-29$ & $5.37 \mathrm{E}-30$ & $6.71 \mathrm{E}-31$ \\
\hline 15.24 & $7.56 \mathrm{E}-30$ & $2.25 \mathrm{E}-30$ & $3.22 \mathrm{E}-31$ \\
\hline 25.40 & $8.55 \mathrm{E}-31$ & $4.36 \mathrm{E}-31$ & $9.50 \mathrm{E}-32$ \\
\hline 30.48 & $3.43 \mathrm{E}-31$ & $1.94 \mathrm{E}-31$ & $5.16 \mathrm{E}-32$ \\
\hline 35.56 & $1.42 \mathrm{E}-31$ & $8.92 \mathrm{E}-32$ & $2.81 \mathrm{E}-32$ \\
\hline
\end{tabular}




\subsection{Karlsruhe Iron Sphere Benchmark Experiment (SBE 6.001)}

a) Experimental Arrangement

The experimental arrangement can be seen on Figure 3.2-1 (taken from Reference 3). Six different iron spheres with diameters of $15,20,25,30,35$ and $40 \mathrm{~cm}$ were investigated. The arrangement was located in a hall, at a minimal distance of $2 \mathrm{~m}$ from the ground and more than $3 \mathrm{~m}$ from the nearest walls. High purity iron (impurities: $\mathrm{C} 0.07 \%, \mathrm{Mn} 0.05 \%, \mathrm{P} 0.009 \%, \mathrm{~S}$ $0.007 \%$ ) was used. The spheres contained a central channel $16 \mathrm{~mm}$ in diameter for housing the source. This channel was filled up with cylindrical pieces made of the same material as the spheres, with the exception of an empty central space $\left(3.57 \mathrm{~cm}^{3}\right)$ provided for the source capsule $\left(2.88 \mathrm{~cm}^{3}\right)$. The source center practically coincides with the sphere center (offset between the two is about $1 \mathrm{~mm}$ ).

Figure 3.2-1: Arrangement of iron sphere experiment.

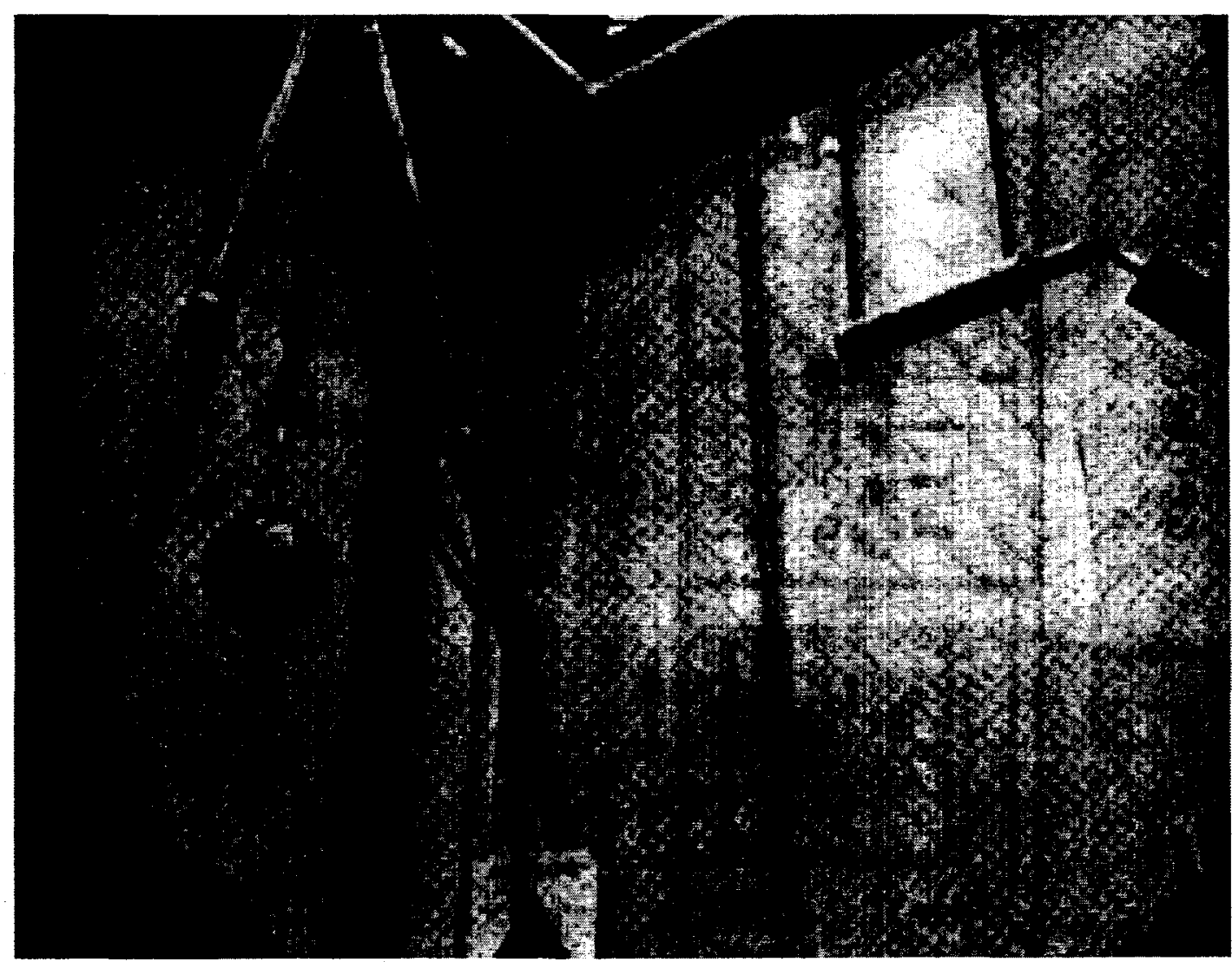


BUGLE 96 VALIDATION WITH MORSE USING WATER AND IRON EXPERIMENTAL DATA FROM SINDBAD 97

WSRC-TR-00349

Rev.0

Page 18 of 89

b) Source Data

A view of the neutron source configuration and its position in the iron sphere is given in Figure 3.2-2. The neutron source consisted of about 30 micrograms of $\mathrm{Cf}-252$ (corresponding to a source strength of $7.0 \mathrm{E}+7 \mathrm{n} / \mathrm{sec})$ deposited on a $\mathrm{SiO}_{2} / \mathrm{MgO}$ matrix $(0.14 \mathrm{~g})$. This matrix was surrounded

Figure 3.2-2: Details of the neutron source.

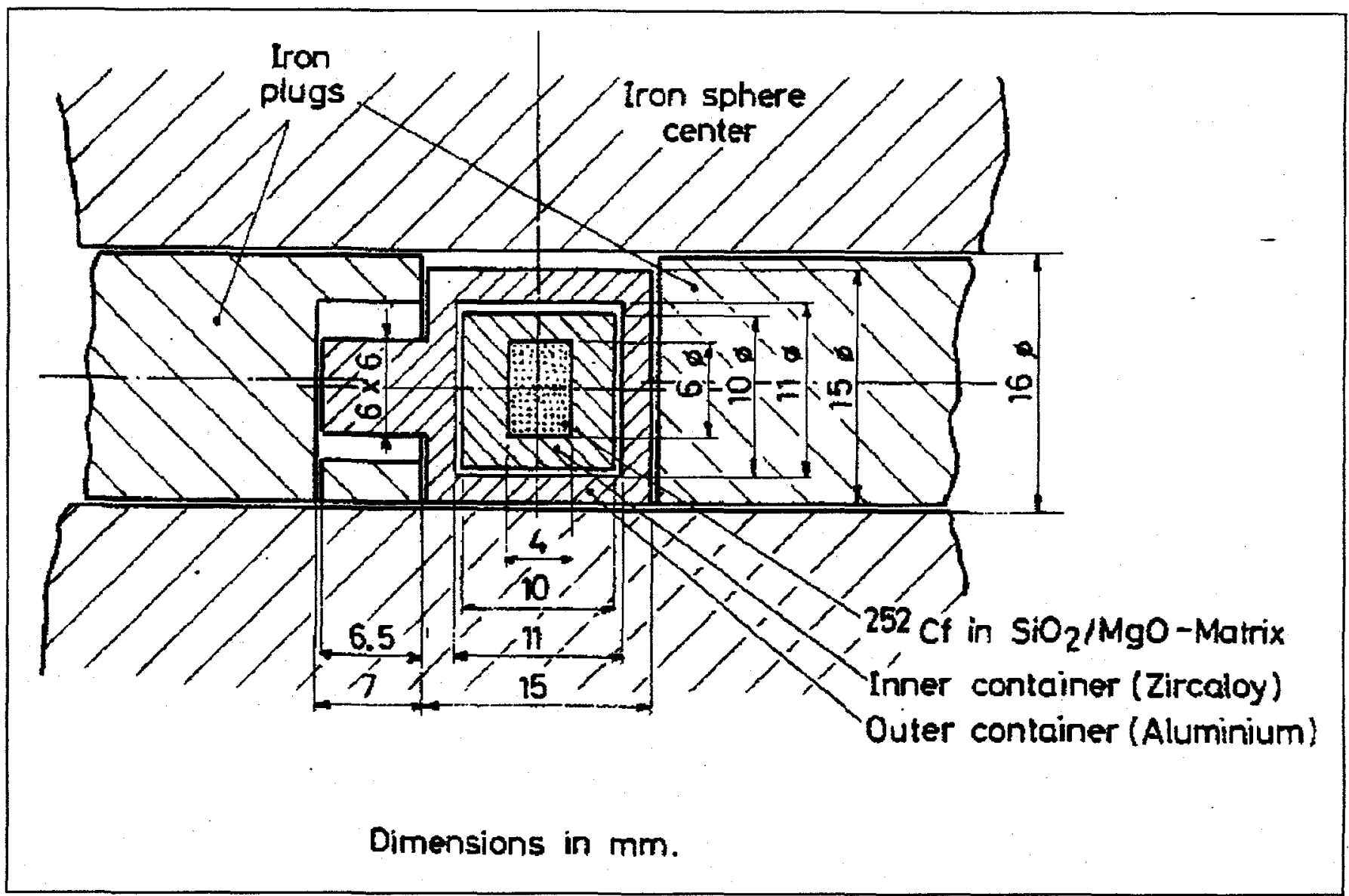

by two capsules; an inner one ( $4.55 \mathrm{~g})$ made of Zircaloy $(\mathrm{Zr}+1.5 \% \mathrm{Sn})$ and an outer one $(4.87 \mathrm{~g})$ made of $\mathrm{AlMg}_{3}(\mathrm{Al}+3 \% \mathrm{Mg})$. The number of atoms per $\mathrm{cm}^{3}$ in the source is compiled in Table $3.2-1$. 
Table 3.2-1: Composition of source capsules for the Karlsruhe Iron Sphere Benchmark Experiment.

\begin{tabular}{|c|c|}
\hline Element & Atom Density (atoms $\left./ \mathrm{cm}^{3}\right)$ \\
\hline $\mathrm{Al}$ & $105.5 \mathrm{E} 21$ \\
\hline $\mathrm{Zr}$ & $29.4 \mathrm{E} 21$ \\
\hline $\mathrm{Mg}$ & $3.9 \mathrm{E} 21$ \\
\hline $\mathrm{O}$ & $2.6 \mathrm{E} 21$ \\
\hline $\mathrm{Si}$ & $0.8 \mathrm{E} 21$ \\
\hline $\mathrm{Sn}$ & $0.4 \mathrm{E} 21$ \\
\hline
\end{tabular}

The neutron source spectrum was measured with proton recoil and He-3 spectrometers. The spectra determined experimentally agree well, but they both deviate rather strongly from the normally assumed Maxwell distribution with average energy $E=2.1 \mathrm{MeV}$. The reason for the deviation has not been explained in the literature [3] we found.

Between 0.9 and $6 \mathrm{MeV}$, the measured source spectrum agrees to within $+/-3 \%$ with the Maxwellian distribution and therefore the calculations can be assumed to be reliable in this energy region. The numerical values of the source spectrum are given in Table 3.2-2 according to the EURLIB [3] group structure.

c) Detectors

\section{Proton recoil $(P R)$ proportional counters}

Three types of detectors were used, the main characteristics of which are given in Table 3.2-3. Precise information about the spectral distribution could be deduced from the pulse height distribution of the cylindrical detectors only if the incident neutrons were nearly parallel to the detector axis. Therefore, the counter axis had to be directed towards the sphere center and the counter center had to be located at distances large in comparison with the sphere radius. The distances between the detectors and the center of the spheres are listed in Table 3.2-4. 
BUGLE 96 VALIDATION WITH MORSE USING WATER

WSRC-TR-00349 AND IRON EXPERIMENTAL DATA FROM SINDBAD 97

Table 3.2-2: Normalized source spectrum for the Cf-252 source in EURLIB energy group structure.

\begin{tabular}{|c|c|c|c|c|c|}
\hline Group & Energy Range $(\mathrm{eV})$ & $\begin{array}{c}\text { Source } \\
\text { Spectrum }\end{array}$ & Group & Energy Range (eV) & $\begin{array}{c}\text { Source } \\
\text { Spectrum }\end{array}$ \\
\hline 1 & 1.3499E07 - 1.4918E07 & $7.07 \mathrm{E}-04$ & 29 & $1.2246 \mathrm{E} 06-1.3534 \mathrm{E} 06$ & $3.60 \mathrm{E}-02$ \\
\hline 2 & 1.2214E07 - 1.3499E07 & $9.39 \mathrm{E}-04$ & 30 & $1.1080 \mathrm{E} 06-1.2246 \mathrm{E} 06$ & $3.35 \mathrm{E}-02$ \\
\hline 3 & $1.1052 \mathrm{E} 07-1.2214 \mathrm{E} 07$ & $1.71 \mathrm{E}-03$ & 31 & $1.0026 \mathrm{E} 06-1.1080 \mathrm{E} 06$ & $3.08 \mathrm{E}-02$ \\
\hline 4 & $1.0000 \mathrm{E} 07-1.1052 \mathrm{E} 07$ & $2.90 \mathrm{E}-03$ & 32 & $9.0718 \mathrm{E} 05-1.0026 \mathrm{E} 06$ & $2.82 \mathrm{E}-02$ \\
\hline 5 & $9.0484 \mathrm{E} 06-1.0000 \mathrm{E} 07$ & $4.61 \mathrm{E}-03$ & 33 & $8.2085 \mathrm{E} 05-9.0718 \mathrm{E} 05$ & $2.57 \mathrm{E}-02$ \\
\hline 6 & $8.1873 \mathrm{E} 06-9.0484 \mathrm{E} 06$ & $6.91 \mathrm{E}-03$ & 34 & $7.4274 \mathrm{E} 05-8.2085 \mathrm{E} 05$ & $2.33 \mathrm{E}-02$ \\
\hline 7 & $7.4082 \mathrm{E} 06-8.1873 \mathrm{E} 06$ & $5.16 \mathrm{E}-03$ & 35 & $6.7206 \mathrm{E} 05-7.4274 \mathrm{E} 05$ & $2.10 \mathrm{E}-02$ \\
\hline 8 & 7.0469E06 - 7.4082E06 & $4.66 \mathrm{E}-03$ & 36 & $6.0810 \mathrm{E} 05-6.7206 \mathrm{E} 05$ & $1.88 \mathrm{E}-02$ \\
\hline 9 & $6.7032 \mathrm{E} 06-7.0469 \mathrm{E} 06$ & $6.54 \mathrm{E}-03$ & 37 & 5.5023E05-6.0810Е05 & $1.68 \mathrm{E}-02$ \\
\hline 10 & $6.3763 \mathrm{E} 06-6.7032 \mathrm{E} 06$ & $6.80 \mathrm{E}-03$ & 38 & 4.9787E05-5.5023Е05 & $1.50 \mathrm{E}-02$ \\
\hline 11 & $6.0653 \mathrm{E} 06-6.3763 \mathrm{E} 06$ & $1.73 \mathrm{E}-02$ & 39 & $4.5049 \mathrm{E} 05-4.9787 \mathrm{E} 05$ & $1.33 \mathrm{E}-\overrightarrow{02}$ \\
\hline 12 & $5.4881 \mathrm{E} 06-6.0653 \mathrm{E} 06$ & $2.17 \mathrm{E}-02$ & 40 & $4.0762 \mathrm{E} 05-4.5049 \mathrm{E} 05$ & $1.18 \mathrm{E}-02$ \\
\hline 13 & 4.9659E06 - 5.4881E06 & $1.11 \mathrm{E}-02$ & 41 & $3.6883 \mathrm{E} 05-4.0762 \mathrm{E} 05$ & $1.04 \mathrm{E}-02$ \\
\hline 14 & 4.7240Е06 - 4.9659Е06 & $1.51 \mathrm{E}-02$ & 42 & $3.3373 \mathrm{E} 05-3.6883 \mathrm{E} 05$ & $9.13 \mathrm{E}-03$ \\
\hline 15 & 4.4933E06 - 4.7240E06 & $3.05 \mathrm{E}-02$ & 43 & 3.0197E05 - 3.3373E05 & $8.02 \mathrm{E}-03$ \\
\hline 16 & 4.0657Е06 - 4.4933Е06 & $3.47 \mathrm{E}-02$ & 44 & 2.7324E05 - 3.0197Е05 & $7.04 \mathrm{E}-03$ \\
\hline 17 & $3.6788 \mathrm{E} 06-4.0657 \mathrm{E} 06$ & $3.83 \mathrm{E}-02$ & 45 & $2.4724 \mathrm{E} 05-2.7324 \mathrm{E} 05$ & $6.16 \mathrm{E}-03$ \\
\hline 18 & $3.3287 \mathrm{E} 06-3.6788 \mathrm{E} 06$ & $4.14 \mathrm{E}-02$ & 46 & $2.2371 \mathrm{E} 05-2.4724 \mathrm{E} 05$ & $5.38 \mathrm{E}-03$ \\
\hline 19 & $3.0112 \mathrm{E} 06-3.3287 \mathrm{E} 06$ & $4.37 \mathrm{E}-02$ & 47 & $2.0242 \mathrm{E} 05-2.2371 \mathrm{E} 05$ & $4.70 \mathrm{E}-03$ \\
\hline 20 & $2.7253 \mathrm{E} 06-3.0112 \mathrm{E} 06$ & $4.53 \mathrm{E}-02$ & 48 & $1.8316 \mathrm{E} 05-2.0242 \mathrm{E} 05$ & $4.09 \mathrm{E}-03$ \\
\hline 21 & $2.4660 \mathrm{E} 06-2.7253 \mathrm{E} 06$ & $2.22 \mathrm{E}-02$ & 49 & $1.6573 \mathrm{E} 05-1.8316 \mathrm{E} 05$ & $3.56 \mathrm{E}-03$ \\
\hline 22 & $2.3460 \mathrm{E} 06-2.4660 \mathrm{E} 06$ & $2.40 \mathrm{E}-02$ & 50 & $1.4996 \mathrm{E} 05-1.6573 \mathrm{E} 05$ & $3.10 \mathrm{E}-03$ \\
\hline 23 & $2.2313 \mathrm{E} 06-2.3460 \mathrm{E} 06$ & $4.62 \mathrm{E}-02$ & 51 & $1.3569 \mathrm{E} 05-1.4996 \mathrm{E} 05$ & $2.69 \mathrm{E}-03$ \\
\hline 24 & $2.0190 \mathrm{E} 06-2.2313 \mathrm{E} 06$ & $4.56 \mathrm{E}-02$ & 52 & $1.2277 \mathrm{E} 05-1.3569 \mathrm{E} 05$ & $2.34 \mathrm{E}-03$ \\
\hline 25 & $1.8268 \mathrm{E} 06-2.0190 \mathrm{E} 06$ & $4.45 \mathrm{E}-02$ & 53 & $1.1109 \mathrm{E} 05-1.2277 \mathrm{E} 05$ & $4.57 \mathrm{E}-03$ \\
\hline 26 & $1.6530 \mathrm{E} 06-1.8268 \mathrm{E} 06$ & $4.28 \mathrm{E}-02$ & 54 & 8.6517E04-1.1109E05 & $3.19 \mathrm{E}-03$ \\
\hline 27 & $1.4957 \mathrm{E} 06-1.6530 \mathrm{E} 06$ & $4.08 \mathrm{E}-02$ & 55 & $6.7379 \mathrm{E} 04-8.6517 \mathrm{E} 04$ & $2.22 \mathrm{E}-03$ \\
\hline 28 & 1.3534E06 - 1.4957E06 & $3.85 E-02$ & & & \\
\hline
\end{tabular}


BUGLE 96 VALIDATION WITH MORSE USING WATER

WSRC-TR-00349

AND IRON EXPERIMENTAL DATA FROM SINDBAD 97

Rev. 0

Page 21 of 89

Table 3.2-3: Recoil proton detector characteristics.

\begin{tabular}{|c|c|c|c|c|c|c|c|}
\hline $\begin{array}{c}\text { Detector } \\
\text { Number }\end{array}$ & $\begin{array}{c}\text { Diameter } \\
(\mathrm{cm})\end{array}$ & $\begin{array}{c}\text { Effective } \\
\text { Length L } \\
(\mathrm{cm})\end{array}$ & $\begin{array}{c}\text { Length of Dead } \\
\text { Volume at Front } \\
(\mathrm{cm})\end{array}$ & $\begin{array}{c}\text { Wall } \\
\text { Thickness } \\
(\mathrm{mm})\end{array}$ & $\begin{array}{c}\text { Gas Fill } \\
(\mathrm{atm})\end{array}$ & $\begin{array}{c}\text { Range } \\
\text { parallel } \\
\text { incidence } \\
(\mathrm{MeV})\end{array}$ & $\begin{array}{c}\text { Energy } \\
\text { Resolution } \\
(\%)\end{array}$ \\
\hline 1 & 4.8 & 12.6 & 4 & 1 & $3 \mathrm{H}_{2}$ & $0.06-0.8$ & 4 \\
\hline 2 & 4.8 & 12.6 & 4 & 1 & $\begin{array}{c}1.5 \mathrm{H}_{2} \\
+1.5 \mathrm{Kr}\end{array}$ & $0.3-2.4$ & 4 \\
\hline 3 & 8.9 & 83.5 & 4 & 2 & $3 \mathrm{CH}_{4}$ & $0.9-5.2$ & 4 \\
\hline
\end{tabular}

Table 3.2-4: Geometry for Leakage Measurements

\begin{tabular}{|c|c|c|c|}
\hline Detector Number & $\mathrm{L}(\mathrm{cm})$ & $\mathrm{D}(\mathrm{cm})$ & $\mathrm{d}(\mathrm{cm})$ \\
\hline 1,2 & 12.6 & 102 & 108 \\
\hline 3 & 83.5 & 117 & 153 \\
\hline
\end{tabular}

$\mathrm{L}$ is the sensitive length and $\mathrm{D}$ the distance between the center of the sphere and the beginning of the sensitive volume;

$$
d=D^{*} \sqrt{1+L / D}
$$

is the distance from the center of the sphere at which a point detector of the same sensitivity would record the same counting rate as the detector actually employed. It is assumed here that the neutron field at the detector position is roughly equivalent to that of a point source in the center of the sphere.

\section{d) Measurement Data}

The results of the proton recoil measurements for each of the three detectors are given in Tables 3.2-5 to 3.2-7. Since the dimensions of detectors 1 and 2 and their arrangement relative to the spheres and the source are the same. The results obtained with detectors 1 and 2 can be related to each other directly without further normalization. The results of detector 3 have been, on the other hand, normalized relative to the results obtained with the other two. This was done by fitting the integral flux value obtained by detector 3 in the range $0.88-1.97 \mathrm{MeV}$ to that of detector 2 , using the mean value of the six sphere measurements and the source spectrum. In the average, the normalization factor of the various measurements departs from the mean of all seven measurements by $+-3 \%$. The error of about $3 \%$ in the absolute values of the flux per source neutron caused by this normalization is restricted to the energy range above $2.4 \mathrm{MeV}$. 
BUGLE 96 VALIDATION WITH MORSE USING WATER AND IRON EXPERIMENTAL DATA FROM SINDBAD 97

WSRC-TR-00349

Rev.0

Page 22 of 89

Table 3.2-5: Detector 1 leakage spectra, proton recoil measurement for iron spheres.

\begin{tabular}{|c|c|c|c|c|c|c|}
\hline \multirow{2}{*}{$\begin{array}{c}\text { Mid energy } \\
(\mathrm{KeV})\end{array}$} & \multicolumn{6}{|c|}{ Detector 1 Measurements (neutrons per source neutron/lethargy unit) } \\
\hline & $\mathrm{d}=15 \mathrm{~cm}$ & $\mathrm{~d}=20 \mathrm{~cm}$ & $\mathrm{~d}=25 \mathrm{~cm}$ & $\mathrm{~d}=30 \mathrm{~cm}$ & $\mathrm{~d}=35 \mathrm{~cm}$ & $\mathrm{~d}=40 \mathrm{~cm}$ \\
\hline $6.325 \mathrm{E}+01$ & $5.302 \mathrm{E}-02$ & 4.173E-02 & 4.319E-02 & 2.163E-02 & $2.606 \mathrm{E}-02$ & 4.318E-02 \\
\hline $6.775 \mathrm{E}+01$ & 3.479E-02 & $3.536 \mathrm{E}-02$ & $5.441 \mathrm{E}-02$ & $3.034 \mathrm{E}-02$ & 3.217E-02 & 4.786E-02 \\
\hline $7.285 \mathrm{E}+01$ & 4.990E-02 & 4.797E-02 & $5.414 \mathrm{E}-02$ & 3.571E-02 & $5.969 \mathrm{E}-02$ & $6.884 \mathrm{E}-02$ \\
\hline $7.850 \mathrm{E}+01$ & $5.576 \mathrm{E}-02$ & $5.941 \mathrm{E}-02$ & $6.054 \mathrm{E}-02$ & $5.095 \mathrm{E}-02$ & $5.925 \mathrm{E}-02$ & 8.025E-02 \\
\hline $8.415 \mathrm{E}+01$ & 4.977E-02 & $5.772 \mathrm{E}-02$ & $5.375 \mathrm{E}-02$ & $5.154 \mathrm{E}-02$ & $6.464 \mathrm{E}-02$ & $9.053 \mathrm{E}-02$ \\
\hline $9.035 \mathrm{E}+01$ & $5.211 \mathrm{E}-02$ & 4.394E-02 & $5.571 \mathrm{E}-02$ & $6.025 \mathrm{E}-02$ & $6.217 \mathrm{E}-02$ & $9.756 \mathrm{E}-02$ \\
\hline $9.710 \mathrm{E}+01$ & $3.674 \mathrm{E}-02$ & $3.965 \mathrm{E}-02$ & $4.266 \mathrm{E}-02$ & $5.023 \mathrm{E}-02$ & $6.333 \mathrm{E}-02$ & $6.874 \mathrm{E}-02$ \\
\hline $1.044 \mathrm{E}+02$ & 4.612E-02 & $5.720 \mathrm{E}-02$ & $5.754 \mathrm{E}-02$ & $5.110 \mathrm{E}-02$ & $6.915 \mathrm{E}-02$ & 8.565E-02 \\
\hline $1.123 \mathrm{E}+02$ & $6.801 \mathrm{E}-02$ & $6.721 \mathrm{E}-02$ & $7.476 \mathrm{E}-02$ & 8.565E-02 & $9.740 \mathrm{E}-02$ & $1.181 \mathrm{E}-01$ \\
\hline $1.208 \mathrm{E}+02$ & $6.462 \mathrm{E}-02$ & $8.190 \mathrm{E}-02$ & $9.185 \mathrm{E}-02$ & 8.609E-02 & $1.298 \mathrm{E}-01$ & $1.415 \mathrm{E}=01$ \\
\hline $1.299 \mathrm{E}+02$ & 7.999E-02 & $1.060 \mathrm{E}-01$ & $1.262 \mathrm{E}-01$ & $1.159 \mathrm{E}-01$ & $1.819 \mathrm{E}-01$ & $1.792 \mathrm{E}-01$ \\
\hline $1.395 \mathrm{E}+02$ & $9.029 \mathrm{E}-02$ & $1.038 \mathrm{E}-01$ & $1.448 \mathrm{E}-01$ & $1.567 \mathrm{E}-01$ & $1.849 \mathrm{E}-01$ & $2.433 \mathrm{E}-01$ \\
\hline $1.496 \mathrm{E}+02$ & 7.504E-02 & $7.228 \mathrm{E}-02$ & $9.407 \mathrm{E}-02$ & $1.382 \mathrm{E}-01$ & $1.528 \mathrm{E}-01$ & $2.006 \mathrm{E}-01$ \\
\hline $1.609 \mathrm{E}+02$ & $6.241 \mathrm{E}-02$ & $9.178 \mathrm{E}-02$ & $9.107 \mathrm{E}-02$ & $1.170 \mathrm{E}-01$ & $1.674 \mathrm{E}-01$ & $1.629 \mathrm{E}-01$ \\
\hline $1.734 E+02$ & $1.140 \mathrm{E}-01$ & $1.300 \mathrm{E}-01$ & $1.630 \mathrm{E}-01$ & $1.567 \mathrm{E}-01$ & $2.096 \mathrm{E}-01$ & $2.342 \mathrm{E}-01$ \\
\hline $1.863 E+02$ & $1.368 \mathrm{E}-01$ & $1.378 \mathrm{E}-01$ & $1.657 \mathrm{E}-01$ & $2.075 \mathrm{E}-01$ & $2.256 \mathrm{E}-01$ & $2.484 \mathrm{E}-01$ \\
\hline $2.004 \mathrm{E}+02$ & $1.072 \mathrm{E}-01$ & $1.240 \mathrm{E}-01$ & $1.383 \mathrm{E}-01$ & $1.829 \mathrm{E}-01$ & $1.645 \mathrm{E}-01$ & $1.924 \mathrm{E}-01$ \\
\hline $2.157 \mathrm{E}+02$ & $1.268 \mathrm{E}-01$ & $1.560 \mathrm{E}-01$ & $1.552 \mathrm{E}-01$ & $1.742 \mathrm{E}-01$ & $2.067 \mathrm{E}-01$ & $1.975 \mathrm{E}-01$ \\
\hline $2.315 \mathrm{E}+02$ & $1.354 \mathrm{E}-01$ & $1.339 \mathrm{E}-01$ & $1.591 \mathrm{E}-01$ & $1.974 \mathrm{E}-01$ & $2.067 \mathrm{E}-01$ & $2.546 \mathrm{E}-01$ \\
\hline $2.484 \mathrm{E}+02$ & $1.226 \mathrm{E}-01$ & $1.625 \mathrm{E}-01$ & $1.670 \mathrm{E}-01$ & $1.974 \mathrm{E}-01$ & $2.475 \mathrm{E}-01$ & $2.658 \mathrm{E}-01$ \\
\hline $2.677 \mathrm{E}+02$ & $1.677 \mathrm{E}-01$ & $2.080 \mathrm{E}-01$ & 2.204E-01 & 2.613E-01 & $3.130 \mathrm{E}-01$ & $3.401 \mathrm{E}-01$ \\
\hline $2.880 \mathrm{E}+02$ & $1.876 \mathrm{E}-01$ & $2.132 \mathrm{E}-01$ & $2.387 \mathrm{E}-01$ & $3.121 \mathrm{E}-01$ & $3.465 \mathrm{E}-01$ & $3.788 \mathrm{E}-01$ \\
\hline $3.095 E+02$ & $2.436 \mathrm{E}-01$ & 2.743E-01 & $3.000 \mathrm{E}-01$ & 3.832E-01 & $4.280 \mathrm{E}-01$ & 4.715E-01 \\
\hline $3.326 \mathrm{E}+02$ & $2.358 \mathrm{E}-01$ & $2.483 \mathrm{E}-01$ & 3.039E-01 & $3.484 \mathrm{E}-01$ & $3.727 \mathrm{E}-01$ & 4.195E-01 \\
\hline $3.575 \mathrm{E}+02$ & $2.788 \mathrm{E}-01$ & $3.250 \mathrm{E}-01$ & $3.457 \mathrm{E}-01$ & $3.788 \mathrm{E}-01$ & $3.814 \mathrm{E}-01$ & $4.318 \mathrm{E}-01$ \\
\hline $3.846 \mathrm{E}+02$ & $2.462 \mathrm{E}-01$ & $2.470 \mathrm{E}-01$ & $2.909 \mathrm{E}-01$ & 3.237E-01 & $2.591 \mathrm{E}-01$ & $3.330 \mathrm{E}-01$ \\
\hline $4.139 \mathrm{E}+02$ & $1.745 \mathrm{E}-01$ & $1.976 \mathrm{E}-01$ & $1.904 \mathrm{E}-01$ & $1.959 \mathrm{E}-01$ & 2.067E-01 & $1.985 \mathrm{E}-01$ \\
\hline $4.450 \mathrm{E}+02$ & $1.980 \mathrm{E}-01$ & $2.418 \mathrm{E}-01$ & $2.439 \mathrm{E}-01$ & $2.279 \mathrm{E}-01$ & $2.911 \mathrm{E}-01$ & $2.454 \mathrm{E}-01$ \\
\hline $4.783 E+02$ & $2.762 \mathrm{E}-01$ & $2.769 \mathrm{E}-01$ & $2.961 \mathrm{E}-01$ & $2.845 \mathrm{E}-01$ & $3.101 \mathrm{E}-01$ & $3.421 \mathrm{E}-01$ \\
\hline $5.144 E+02$ & $2.462 \mathrm{E}-01$ & $2.613 \mathrm{E}-01$ & $2.805 \mathrm{E}-01$ & $3.077 \mathrm{E}-01$ & $3.130 \mathrm{E}-01$ & $3.279 \mathrm{E}-01$ \\
\hline $5.534 \mathrm{E}+02$ & $3.087 \mathrm{E}-01$ & $3.445 \mathrm{E}-01$ & $3.470 \mathrm{E}-01$ & 3.788E-01 & $4.120 \mathrm{E}-01$ & $3.992 \mathrm{E}-01$ \\
\hline $5.952 E+02$ & $4.012 \mathrm{E}-01$ & $4.537 \mathrm{E}-01$ & $4.840 \mathrm{E}-01$ & $5.313 \mathrm{E}-01$ & $6.435 \mathrm{E}-01$ & $5.906 \mathrm{E}-01$ \\
\hline $6.398 \mathrm{E}+02$ & $4.273 E-01$ & $4.888 \mathrm{E}-01$ & $5.636 \mathrm{E}-01$ & $5.995 \mathrm{E}-01$ & $5.226 \mathrm{E}-01$ & $6.110 \mathrm{E}-01$ \\
\hline $6.878 E+02$ & $3.817 \mathrm{E}-01$ & $4.290 \mathrm{E}-01$ & 4.540E-01 & 4.674E-01 & $3.930 \mathrm{E}-01$ & $4.440 \mathrm{E}-01$ \\
\hline $7.392 \mathrm{E}+02$ & $4.038 \mathrm{E}-01$ & $3.757 \mathrm{E}-01$ & 3.809E-01 & $3.527 \mathrm{E}-01$ & $3.261 \mathrm{E}-01$ & $2.820 \mathrm{E}-01$ \\
\hline $7.951 E+02$ & $3.960 \mathrm{E}-01$ & 3.432E-01 & $3.561 \mathrm{E}-01$ & $3.527 \mathrm{E}-01$ & $3.261 \mathrm{E}-01$ & $3.218 \mathrm{E}-01$ \\
\hline $8.550 \mathrm{E}+02$ & $2.840 \mathrm{E}-01$ & $3.341 \mathrm{E}-01$ & $3.261 \mathrm{E}-01$ & $3.121 \mathrm{E}-01$ & $2.970 \mathrm{E}-01$ & 2.932E-01 \\
\hline
\end{tabular}


BUGLE 96 VALIDATION WITH MORSE USING WATER AND IRON EXPERIMENTAL DATA FROM SINDBAD 97

WSRC-TR-00349

Rev.0

Page 23 of 89

Table 3.2-6: Detector 2 leakage spectra, proton recoil measurements for iron spheres.

\begin{tabular}{|c|c|c|c|c|c|c|}
\hline \multirow{2}{*}{$\begin{array}{c}\text { Mid energy } \\
(\mathrm{KeV})\end{array}$} & \multicolumn{6}{|c|}{ Detector 2 Measurements (neutrons per source neutron/lethargy unit) } \\
\hline & $\mathrm{d}=15 \mathrm{~cm}$ & $\mathrm{~d}=20 \mathrm{~cm}$ & $\mathrm{~d}=25 \mathrm{~cm}$ & $\mathrm{~d}=30 \mathrm{~cm}$ & $\mathrm{~d}=35 \mathrm{~cm}$ & $\mathrm{~d}=40 \mathrm{~cm}$ \\
\hline $3.081 E+02$ & $2.275 \mathrm{E}-01$ & $3.339 \mathrm{E}-01$ & $3.145 \mathrm{E}-01$ & 3.572E-01 & 4.349E-01 & $4.417 \mathrm{E}-01$ \\
\hline $3.317 \mathrm{E}+02$ & $2.448 \mathrm{E}-01$ & 3.051E-01 & 3.202E-01 & $3.445 \mathrm{E}-01$ & $3.983 \mathrm{E}-01$ & 3.784E-01 \\
\hline $3.568 \mathrm{E}+02$ & $2.592 \mathrm{E}-01$ & $2.965 \mathrm{E}-01$ & $3.490 \mathrm{E}-01$ & $3.728 \mathrm{E}-01$ & 4.223E-01 & $4.149 \mathrm{E}-01$ \\
\hline $3.833 \mathrm{E}+02$ & 2.117E-01 & $2.792 \mathrm{E}-01$ & $2.886 \mathrm{E}-01$ & $2.693 \mathrm{E}-01$ & $3.139 \mathrm{E}-01$ & $3.108 \mathrm{E}-01$ \\
\hline $4.110 \mathrm{E}+02$ & $1.786 \mathrm{E}-01$ & $1.971 \mathrm{E}-01$ & $2.140 \mathrm{E}-01$ & $2.084 \mathrm{E}-01$ & $2.181 \mathrm{E}-01$ & $2.278 \mathrm{E}-01$ \\
\hline $4.423 \mathrm{E}+02$ & $1.858 \mathrm{E}-01$ & $2.432 \mathrm{E}-01$ & $2.398 \mathrm{E}-01$ & $2.623 \mathrm{E}-01$ & $2.576 \mathrm{E}-01$ & $2.799 \mathrm{E}-01$ \\
\hline $4.762 \mathrm{E}+02$ & $2.751 \mathrm{E}-01$ & $2.835 \mathrm{E}-01$ & $3.231 \mathrm{E}-01$ & $3.289 \mathrm{E}-01$ & $3.125 \mathrm{E}-01$ & $3.249 \mathrm{E}-01$ \\
\hline $5.130 \mathrm{E}+02$ & $2.837 \mathrm{E}-01$ & $2.821 \mathrm{E}-01$ & $3.073 \mathrm{E}-01$ & $3.019 \mathrm{E}-01$ & 3.392E-01 & $3.277 \mathrm{E}-01$ \\
\hline $5.514 \mathrm{E}+02$ & $2.895 \mathrm{E}-01$ & 3.296E-01 & $3.561 \mathrm{E}-01$ & $3.799 \mathrm{E}-01$ & $3.800 \mathrm{E}-01$ & $4.065 \mathrm{E}-01$ \\
\hline $5.927 \mathrm{E}+02$ & $3.586 \mathrm{E}-01$ & 4.145E-01 & 4.423E-01 & $5.019 \mathrm{E}-01$ & $5.574 \mathrm{E}-01$ & $5.851 \mathrm{E}=01$ \\
\hline $6.369 E+02$ & $4.709 \mathrm{E}-01$ & $5.109 \mathrm{E}-01$ & $5.874 \mathrm{E}-01$ & $6.110 \mathrm{E}-01$ & $6.447 \mathrm{E}-01$ & $6.259 \mathrm{E}-01$ \\
\hline $6.840 \mathrm{E}+02$ & $5.905 \mathrm{E}-01$ & $4.346 \mathrm{E}-01$ & $4.667 \mathrm{E}-01$ & $4.225 \mathrm{E}-01$ & $5.391 \mathrm{E}-01$ & $4.009 \mathrm{E}-01$ \\
\hline $7.356 \mathrm{E}+02$ & $3.082 \mathrm{E}-01$ & $3.713 \mathrm{E}-01$ & $3.461 \mathrm{E}-01$ & $3.516 \mathrm{E}-01$ & $3.519 \mathrm{E}-01$ & $3.418 \mathrm{E}-01$ \\
\hline $7.917 \mathrm{E}+02$ & $3.456 \mathrm{E}-01$ & $3.353 \mathrm{E}-01$ & $3.346 \mathrm{E}-01$ & $3.204 \mathrm{E}-01$ & $3.195 \mathrm{E}-01$ & $3.094 \mathrm{E}-01$ \\
\hline $8.521 E+02$ & 4.004E-01 & $3.396 \mathrm{E}-01$ & $3.418 \mathrm{E}-01$ & $3.204 \mathrm{E}-01$ & $3.068 \mathrm{E}-01$ & $2.560 \mathrm{E}-01$ \\
\hline $9.155 \mathrm{E}+02$ & 3.672E-01 & $3.799 \mathrm{E}-01$ & $3.604 \mathrm{E}-01$ & $3.558 \mathrm{E}-01$ & $3.505 \mathrm{E}-01$ & $3.263 \mathrm{E}-01$ \\
\hline $9.850 \mathrm{E}+02$ & $3.629 \mathrm{E}-01$ & $3.742 \mathrm{E}-01$ & $3.533 \mathrm{E}-01$ & $3.615 \mathrm{E}-01$ & $3.181 \mathrm{E}-01$ & $2.968 \mathrm{E}-01$ \\
\hline $1.060 \mathrm{E}+03$ & $3.860 \mathrm{E}-01$ & $3.627 \mathrm{E}-01$ & $3.245 \mathrm{E}-01$ & $2.864 \mathrm{E}-01$ & $3.068 \mathrm{E}-01$ & $2.349 \mathrm{E}-01$ \\
\hline $1.139 \mathrm{E}+03$ & $4.033 \mathrm{E}-01$ & $3.727 \mathrm{E}-01$ & $3.691 \mathrm{E}-01$ & $3.317 \mathrm{E}-01$ & $3.209 \mathrm{E}-01$ & $2.757 \mathrm{E}-01$ \\
\hline $1.225 \mathrm{E}+03$ & $3.384 \mathrm{E}-01$ & $3.468 \mathrm{E}-01$ & 3.533E-01 & $2.991 \mathrm{E}-01$ & $2.463 \mathrm{E}-01$ & $2.067 \mathrm{E}-01$ \\
\hline $1.316 \mathrm{E}+03$ & $3.730 \mathrm{E}-01$ & $3.612 \mathrm{E}-01$ & $3.202 \mathrm{E}-01$ & $2.778 \mathrm{E}-01$ & $2.322 \mathrm{E}-01$ & $1.730 \mathrm{E}-01$ \\
\hline $1.415 E+03$ & $3.888 \mathrm{E}-01$ & $3.382 \mathrm{E}-01$ & $2.743 \mathrm{E}-01$ & $2.013 \mathrm{E}-01$ & $2.111 \mathrm{E}-01$ & $1.547 \mathrm{E}-01$ \\
\hline $1.523 \mathrm{E}+03$ & $3.485 \mathrm{E}-01$ & $3.152 \mathrm{E}-01$ & $2.484 \mathrm{E}-01$ & $2.282 \mathrm{E}-01$ & $1.858 \mathrm{E}-01$ & $1.399 \mathrm{E}-01$ \\
\hline $1.638 E+03$ & $4.076 \mathrm{E}-01$ & $3.353 \mathrm{E}-01$ & $2.743 \mathrm{E}-01$ & $2.041 \mathrm{E}-01$ & $1.731 \mathrm{E}-01$ & $1.448 \mathrm{E}-01$ \\
\hline $1.760 \mathrm{E}+03$ & $3.269 \mathrm{E}-01$ & $2.677 \mathrm{E}-01$ & $2.369 \mathrm{E}-01$ & $1.999 \mathrm{E}-01$ & $1.618 \mathrm{E}-01$ & $1.129 \mathrm{E}-01$ \\
\hline $1.893 E+03$ & $3.269 \mathrm{E}-01$ & $3.137 \mathrm{E}-01$ & $2.355 \mathrm{E}-01$ & $1.531 \mathrm{E}-01$ & $1.141 \mathrm{E}-01$ & $9.790 \mathrm{E}-02$ \\
\hline $2.036 \mathrm{E}+03$ & $2.578 \mathrm{E}-01$ & $2.029 \mathrm{E}-01$ & $1.795 \mathrm{E}-01$ & $1.355 \mathrm{E}-01$ & $9.769 \mathrm{E}-02$ & $7.132 \mathrm{E}-02$ \\
\hline $2.189 E+03$ & $2.621 \mathrm{E}-01$ & $1.885 \mathrm{E}-01$ & $1.565 \mathrm{E}-01$ & $1.273 \mathrm{E}-01$ & $1.009 \mathrm{E}-01$ & $6.400 \mathrm{E}-02$ \\
\hline $2.354 \mathrm{E}+03$ & $2.318 \mathrm{E}-01$ & $2.302 \mathrm{E}-01$ & $1.317 \mathrm{E}-01$ & $9.669 \mathrm{E}-02$ & $7.348 \mathrm{E}-02$ & $5.205 \mathrm{E}-02$ \\
\hline
\end{tabular}


BUGLE 96 VALIDATION WITH MORSE USING WATER

AND IRON EXPERIMENTAL DATA FROM SINDBAD 97

WSRC-TR-00349

Rev.0

Page 24 of 89

Table 3.2-7: Detector 3 leakage spectra, proton recoil measurements for iron spheres.

\begin{tabular}{|c|c|c|c|c|c|c|}
\hline \multirow{2}{*}{$\begin{array}{l}\text { Mid energy } \\
(\mathrm{KeV})\end{array}$} & \multicolumn{6}{|c|}{ Detector 3 Measurements (neutrons per source neutron/lethargy unit) } \\
\hline & $\mathrm{d}=15 \mathrm{~cm}$ & $\mathrm{~d}=20 \mathrm{~cm}$ & $\mathrm{~d}=25 \mathrm{~cm}$ & $\mathrm{~d}=30 \mathrm{~cm}$ & $\mathrm{~d}=35 \mathrm{~cm}$ & $\mathrm{~d}=40 \mathrm{~cm}$ \\
\hline $9.165 \mathrm{E}+02$ & 3.811E-01 & 3.643E-01 & 3.655E-01 & 3.682E-01 & 3.519E-01 & 3.247E-01 \\
\hline $9.870 \mathrm{E}+02$ & 3.853E-01 & 3.811E-01 & 3.674E-01 & 3.703E-01 & 3.413E-01 & 3.205E-01 \\
\hline $1.063 \mathrm{E}+03$ & 3.769E-01 & 3.685E-01 & $3.270 \mathrm{E}-01$ & $3.114 \mathrm{E}-01$ & $2.845 \mathrm{E}-01$ & 2.551E-01 \\
\hline $1.142 \mathrm{E}+03$ & $4.001 \mathrm{E}-01$ & $3.938 \mathrm{E}-01$ & $3.694 \mathrm{E}-01$ & $3.430 \mathrm{E}-01$ & $3.055 \mathrm{E}-01$ & $2.678 \mathrm{E}-01$ \\
\hline $1.226 \mathrm{E}+03$ & 4.169E-01 & $3.938 \mathrm{E}-01$ & $3.578 \mathrm{E}-01$ & $3.367 \mathrm{E}-01$ & $2.992 \mathrm{E}-01$ & $2.572 E-01$ \\
\hline $1.320 \mathrm{E}+03$ & $3.769 \mathrm{E}-01$ & $3.474 \mathrm{E}-01$ & $3.001 \mathrm{E}-01$ & 2.693E-01 & $2.276 \mathrm{E}-01$ & $1.927 \mathrm{E}-01$ \\
\hline $1.419 \mathrm{E}+03$ & 3.706E-01 & $3.222 \mathrm{E}-01$ & 2.789E-01 & $2.399 \mathrm{E}-01$ & $1.968 \mathrm{E}-01$ & $1.607 \mathrm{E}-01$ \\
\hline $1.524 \mathrm{E}+03$ & $3.453 \mathrm{E}-01$ & $2.969 \mathrm{E}-01$ & $2.539 \mathrm{E}-01$ & $2.104 \mathrm{E}-01$ & $1.713 \mathrm{E}-01$ & $1.415 \mathrm{E}-01$ \\
\hline $1.638 \mathrm{E}+03$ & 3.537E-01 & 2.990E-01 & $2.482 \mathrm{E}-01$ & $1.986 \mathrm{E}-01$ & $1.656 \mathrm{E}-01$ & 1.282E-01 \\
\hline $1.761 E+03$ & $3.601 \mathrm{E}-01$ & $2.990 \mathrm{E}-01$ & $2.501 \mathrm{E}-01$ & $2.056 \mathrm{E}-01$ & $1.635 \mathrm{E}-01$ & $1.282 \mathrm{E}=01$ \\
\hline $1.896 \mathrm{E}+03$ & $3.348 \mathrm{E}-01$ & $2.716 \mathrm{E}-01$ & $2.193 \mathrm{E}-01$ & $1.742 \mathrm{E}-01$ & 1.365E-01 & $1.031 \mathrm{E}-01$ \\
\hline $2.039 E+03$ & $2.906 \mathrm{E}-01$ & 2.274E-01 & $1.797 \mathrm{E}-01$ & $1.348 \mathrm{E}-01$ & $1.051 \mathrm{E}-01$ & 8.098E-02 \\
\hline $2.191 E+03$ & $2.842 \mathrm{E}-01$ & $2.253 \mathrm{E}-01$ & $1.737 \mathrm{E}-01$ & $1.302 \mathrm{E}-01$ & $9.905 \mathrm{E}-02$ & $7.255 \mathrm{E}-02$ \\
\hline $2.355 \mathrm{E}+03$ & $2.779 \mathrm{E}-01$ & $2.148 \mathrm{E}-01$ & $1.622 \mathrm{E}-01$ & $1.256 \mathrm{E}-01$ & $8.830 \mathrm{E}-02$ & 6.137E-02 \\
\hline $2.533 E+03$ & $2.484 \mathrm{E}-01$ & $1.874 \mathrm{E}-01$ & $1.373 \mathrm{E}-01$ & $9.890 \mathrm{E}-02$ & $7.186 \mathrm{E}-02$ & $5.272 \mathrm{E}-02$ \\
\hline $2.726 \mathrm{E}+03$ & 2.337E-01 & 1.667E-01 & $1.254 \mathrm{E}-01$ & 8.901E-02 & $6.385 \mathrm{E}-02$ & $4.408 E-02$ \\
\hline $2.931 \mathrm{E}+03$ & $2.076 \mathrm{E}-01$ & $1.480 \mathrm{E}-01$ & $1.098 \mathrm{E}-01$ & $8.060 \mathrm{E}-02$ & $5.585 \mathrm{E}-02$ & $3.901 \mathrm{E}-02$ \\
\hline $3.150 \mathrm{E}+03$ & $1.916 \mathrm{E}-01$ & $1.373 \mathrm{E}-01$ & $9.755 \mathrm{E}-02$ & 6.797E-02 & 4.594E-02 & $3.227 \mathrm{E}-02$ \\
\hline $3.387 E+03$ & $1.619 \mathrm{E}-01$ & $1.162 \mathrm{E}-01$ & 8.389E-02 & $5.871 \mathrm{E}-02$ & 4.257E-02 & 2.784E-02 \\
\hline $3.641 E+03$ & $1.453 \mathrm{E}-01$ & $1.000 \mathrm{E}-01$ & 7.234E-02 & $4.840 \mathrm{E}-02$ & $3.161 \mathrm{E}-02$ & $2.320 \mathrm{E}-02$ \\
\hline $3.916 \mathrm{E}+03$ & 1.303E-01 & $8.108 \mathrm{E}-02$ & $6.234 \mathrm{E}-02$ & $3.977 \mathrm{E}-02$ & 2.613E-02 & $1.696 \mathrm{E}-02$ \\
\hline $4.212 E+03$ & $1.128 \mathrm{E}-01$ & $8.276 \mathrm{E}-02$ & $5.445 \mathrm{E}-02$ & 3.409E-02 & 2.297E-02 & $1.527 \mathrm{E}-02$ \\
\hline $4.527 \mathrm{E}+03$ & $8.739 \mathrm{E}-02$ & $5.328 \mathrm{E}-02$ & 4.117E-02 & $2.862 \mathrm{E}-02$ & $1.795 \mathrm{E}-02$ & $1.284 \mathrm{E}-02$ \\
\hline $4.869 \mathrm{E}+03$ & $6.970 \mathrm{E}-02$ & 4.801E-02 & $3.598 \mathrm{E}-02$ & $2.125 \mathrm{E}-02$ & $1.385 \mathrm{E}-02$ & 8.220E-03 \\
\hline $5.238 \mathrm{E}+03$ & 4.843E-02 & $2.864 \mathrm{E}-02$ & $2.386 \mathrm{E}-02$ & $1.290 \mathrm{E}-02$ & $9.310 \mathrm{E}-03$ & $6.810 \mathrm{E}-03$ \\
\hline
\end{tabular}




\subsection{Benchmark Model Description}

\subsection{Winfrith Water Benchmark Model}

Although the source used in the original experiment was Cf-252, it was replaced by U-238 in the material section for the calculations in this document. This approach was necessary since neither of the BUGLE cross section libraries utilized had Cf- 252 as one of its nuclides. After all, the Cf252 spectrum provided in Reference 3 was imposed to account for more accurate results.

The source-detector arrangement was modeled quite similar to that shown in Figure 3.1-2. The sulfur sample region, as well as the aluminum tube in which it is contained, were modeled as infinite cylinders. For simplicity, the arms of the support structure were not modeled in this experiment.

The outer stainless-steel source capsule was modeled as a cylinder of infinite length with a radius of $0.95 \mathrm{~cm}$. The modeling of the capsule's neck was considered negligible due to the conservative radius chosen. The small amount of air and aluminum in its central region were also neglected.

The inner source capsule walls of the container holding the Cf- 252 source were not represented in the model.

The whole source-detector arrangement was treated as being immersed in an infinite bath of water. This was considered a conservative assumption since the nearest distance from the source to an external boundary was more than 4 times the migration length of a $5 \mathrm{MeV}$ neutron in water. 


\subsection{Karlsruhe Iron Sphere Benchmark Model}

The model for this experiment consisted of the iron sphere containing the source and the proton recoil (PR) proportional counters positioned around it. For simplicity, the support structure and the wires that held the sphere in place were not modeled.

The source capsule inside the iron sphere was modeled much like the one depicted in Figure 3.22 with the following two exceptions. First, the zirconium content of the inner capsule surrounding the source was replaced by aluminium. This was a necessary approach due to the lack of zirconium as part of the BUGLE cross section libraries utilized in this experiment. Second, the capsules neck was considered negligible due to its small radius. The void to be left by the capsule neck was filled with the same material as that of the sphere. 
BUGLE 96 VALIDATION WITH MORSE USING WATER

WSRC-TR-00349

AND IRON EXPERIMENTAL DATA FROM SINDBAD 97

Rev.0

Page 27 of 89

\subsection{Results}

\subsection{Winfrith Water Benchmark Experiment}

Fast neutron spectrum measurement data made with a NE-213 organic liquid scintillator within the hollow tube at the axial midplane for each of the seven source locations along with the calculated values are shown in Tables 5.1-1 to 5.1-21. Size of water tank and the placement of sources and detectors make the essential portions of the calculational geometry two-dimensional.

Figures 5.1-1 to 5.1-7 give a graphical comparison of the ratio of the calculated (C) and experimental (E) detector readings using both BUGLE-96 and BUGLE-80 cross section libraries. The experimental values are read as 1.0 on the $\mathrm{C} / \mathrm{E}$ axis.

The $\mathrm{C} / \mathrm{E}$ ratios are plotted against neutron energies varying from $\sim 1 \mathrm{MeV}$ to $10 \mathrm{MeV}$. For a detector at a distance of $10.16 \mathrm{~cm}$ from the source, the calculated values were within $15 \%$ of the measured values from $1 \mathrm{MeV}$ to about $6 \mathrm{MeV}$. From $6 \mathrm{MeV}$ to about $8 \mathrm{MeV}$, the calculated flux was much higher by as much as $30 \%$. A dip in the calculated value around $3.6 \mathrm{MeV}$ was noticed, where the calculated flux was underpredicted by about $25 \%$. The calculated $\mathrm{C} / \mathrm{E}$ ratios for BUGLE-96 set were consistent and within 5\% of the BUGLE-80 values. In general, the calculated values were underpredicted in the energy band except near the high energy range above $6 \mathrm{MeV}$.

The trend was similar for other detector distances. At a higher detector distances of $30.56 \mathrm{~cm}$, the differences between the C/E ratios for BUGLE-96 and BUGLE-80 set were increased at energy above $\sim 3 \mathrm{MeV}$, where the calculated flux with BUGLE-96 were generally lower and those with BUGLE-80 were generally higher than the corresponding experimental results.

In summary, the neutron flux calculated with BUGLE-96 matched well (within $15 \%-20 \%$ ) with the experimental value over $2 \mathrm{MeV}$ to $8 \mathrm{MeV}$ range. There was not much overall improvement with the BUGLE-96 cross section set over the corresponding BUGLE-80 set, and the trend for both cases was quite similar. 
BUGLE 96 VALIDATION WITH MORSE USING WATER

WSRC-TR-00349 AND IRON EXPERIMENTAL DATA FROM SINDBAD 97

Rev.0

Page 28 of 89

Table 5.1-1: BUGLE 80 results for a detector at a distance of $10.16 \mathrm{~cm}$ from source.

\begin{tabular}{|c|c|c|c|c|}
\hline \multirow[b]{2}{*}{ Group } & \multirow[b]{2}{*}{$\begin{array}{c}\text { Energy boundary } \\
(\mathrm{MeV})\end{array}$} & \multicolumn{2}{|c|}{ Flux/Lethargy per Source Neutron } & \multirow[b]{2}{*}{$\mathrm{C} / \mathrm{E}$} \\
\hline & & $\begin{array}{c}\text { Calculated (C) } \\
\left(\mathrm{cm}^{-2} / \mathrm{s}\right)\end{array}$ & $\begin{array}{c}\text { Experimental (E) } \\
\left(\mathrm{cm}^{-2} / \mathrm{s}\right)\end{array}$ & \\
\hline 20 & 10 & & & \\
\hline 19 & 8.825 & $1.090 \mathrm{E}-05$ & $6.770 \mathrm{E}-06$ & 1.61 \\
\hline 18 & 7.788 & $2.024 \mathrm{E}-05$ & $1.650 \mathrm{E}-05$ & 1.23 \\
\hline 17 & 6.873 & $3.542 \mathrm{E}-05$ & $2.750 \mathrm{E}-05$ & 1.29 \\
\hline 16 & 6.065 & $4.070 \mathrm{E}-05$ & $4.620 \mathrm{E}-05$ & 0.88 \\
\hline 15 & 5.353 & $7.492 \mathrm{E}-05$ & $6.660 \mathrm{E}-05$ & 1.12 \\
\hline 14 & 4.724 & $9.022 \mathrm{E}-05$ & $9.150 \mathrm{E}-05$ & 0.99 \\
\hline 13 & 4.169 & $1.136 \mathrm{E}-04$ & $1.060 \mathrm{E}-04$ & 1.07 \\
\hline 12 & 3.679 & $1.003 \mathrm{E}-04$ & $1.350 \mathrm{E}-04$ & 0.74 \\
\hline 11 & 3.247 & $1.573 \mathrm{E}-04$ & $1.450 \mathrm{E}-04$ & 1.09 \\
\hline 10 & 2.865 & $1.627 \mathrm{E}-04$ & $1.790 \mathrm{E}-04$ & 0.91 \\
\hline 9 & 2.528 & $1.936 \mathrm{E}-04$ & $2.080 \mathrm{E}-04$ & 0.93 \\
\hline 8 & 2.231 & $2.113 \mathrm{E}-04$ & $2.130 \mathrm{E}-04$ & 0.99 \\
\hline 7 & 1.969 & $1.948 \mathrm{E}-04$ & $2.100 \mathrm{E}-04$ & 0.93 \\
\hline 6 & 1.738 & $1.940 \mathrm{E}-04$ & $1.980 \mathrm{E}-04$ & 0.98 \\
\hline 5 & 1.534 & $1.925 \mathrm{E}-04$ & $1.980 \mathrm{E}-04$ & 0.97 \\
\hline 4 & 1.353 & $1.801 \mathrm{E}-04$ & $2.020 \mathrm{E}-04$ & 0.89 \\
\hline 3 & 1.194 & $1.824 \mathrm{E}-04$ & $1.800 \mathrm{E}-04$ & 1.01 \\
\hline 2 & 1.054 & $1.610 \mathrm{E}-04$ & $1.850 \mathrm{E}-04$ & 0.87 \\
\hline 1 & 0.930 & $1.557 \mathrm{E}-04$ & $1.600 \mathrm{E}-04$ & 0.97 \\
\hline
\end{tabular}


BUGLE 96 VALIDATION WITH MORSE USING WATER

WSRC-TR-00349

AND IRON EXPERIMENTAL DATA FROM SINDBAD 97

Rev.0

Page 29 of 89

Table 5.1-2: BUGLE 96 results for a detector at a distance of $10.16 \mathrm{~cm}$ from source.

\begin{tabular}{|c|c|c|c|c|}
\hline \multirow[b]{2}{*}{ Group } & \multirow[b]{2}{*}{$\begin{array}{c}\text { Energy boundary } \\
(\mathrm{MeV})\end{array}$} & \multicolumn{2}{|c|}{ Flux/Lethargy per Source Neutron } & \multirow[b]{2}{*}{$\mathrm{C} / \mathrm{E}$} \\
\hline & & $\begin{array}{c}\text { Calculated (C) } \\
\left(\mathrm{cm}^{-2} / \mathrm{s}\right)\end{array}$ & $\begin{array}{l}\text { Experimental (E) } \\
\left(\mathrm{cm}^{-2} / \mathrm{s}\right)\end{array}$ & \\
\hline 20 & 10 & & & \\
\hline 19 & 8.825 & $1.195 \mathrm{E}-05$ & $6.770 \mathrm{E}-06$ & 1.76 \\
\hline 18 & 7.788 & $1.988 \mathrm{E}-05$ & $1.650 \mathrm{E}-05$ & 1.20 \\
\hline 17 & 6.873 & $3.573 \mathrm{E}-05$ & $2.750 \mathrm{E}-05$ & 1.30 \\
\hline 16 & 6.065 & $4.156 \mathrm{E}-05$ & $4.620 \mathrm{E}-05$ & 0.90 \\
\hline 15 & 5.353 & $7.600 \mathrm{E}-05$ & $6.660 \mathrm{E}-05$ & 1.14 \\
\hline 14 & 4.724 & $9.141 \mathrm{E}-05$ & $9.150 \mathrm{E}-05$ & 1.00 \\
\hline 13 & 4.169 & $1.150 \mathrm{E}-04$ & $1.060 \mathrm{E}-04$ & 1.09 \\
\hline 12 & 3.679 & $1.015 \mathrm{E}-04$ & $1.350 \mathrm{E}-04$ & 0.75 \\
\hline 11 & 3.247 & $1.456 \mathrm{E}-04$ & $1.450 \mathrm{E}-04$ & 1.00 \\
\hline 10 & 2.865 & $1.547 \mathrm{E}-04$ & $1.790 \mathrm{E}-04$ & 0.86 \\
\hline 9 & 2.528 & $1.963 \mathrm{E}-04$ & $2.080 \mathrm{E}-04$ & 0.94 \\
\hline 8 & 2.231 & $2.086 \mathrm{E}-04$ & $2.130 \mathrm{E}-04$ & 0.98 \\
\hline 7 & 1.969 & $2.019 \mathrm{E}-04$ & $2.100 \mathrm{E}-04$ & 0.96 \\
\hline 6 & 1.738 & $1.983 \mathrm{E}-04$ & $1.980 \mathrm{E}-04$ & 1.00 \\
\hline 5 & 1.534 & $1.934 \mathrm{E}-04$ & $1.980 \mathrm{E}-04$ & 0.98 \\
\hline 4 & 1.353 & $1.792 \mathrm{E}-04$ & $2.020 \mathrm{E}-04$ & 0.89 \\
\hline 3 & 1.194 & $1.852 \mathrm{E}-04$ & $1.800 \mathrm{E}-04$ & 1.03 \\
\hline 2 & 1.054 & $1.634 \mathrm{E}-04$ & $1.850 \mathrm{E}-04$ & 0.88 \\
\hline 1 & 0.930 & $1.554 \mathrm{E}-04$ & $1.600 \mathrm{E}-04$ & 0.97 \\
\hline
\end{tabular}


BUGLE 96 VALIDATION WITH MORSE USING WATER

WSRC-TR-00349

AND IRON EXPERIMENTAL DATA FROM SINDBAD 97

Rev.0

Page 30 of 89

Table 5.1-3: BUGLE 80 and BUGLE 96 comparison. Detector at $10.16 \mathrm{~cm}$ from source.

\begin{tabular}{|c|c|c|c|}
\hline Group & Energy Boundary & \multicolumn{2}{|c|}{ Calculated/Experimental } \\
\hline & $(\mathrm{MeV})$ & BUGLE 80 & BUGLE 96 \\
\hline 20 & 10 & & \\
19 & 8.825 & 1.61 & 1.76 \\
18 & 7.788 & 1.23 & 1.20 \\
17 & 6.873 & 1.29 & 1.30 \\
16 & 6.065 & 0.88 & 0.90 \\
15 & 5.353 & 1.12 & 1.14 \\
14 & 4.724 & 0.99 & 1.00 \\
13 & 4.169 & 1.07 & 1.09 \\
12 & 3.679 & 0.74 & 0.75 \\
11 & 3.247 & 1.09 & 1.00 \\
10 & 2.865 & 0.91 & 0.86 \\
9 & 2.528 & 0.93 & 0.94 \\
8 & 2.231 & 0.99 & 0.98 \\
7 & 1.969 & 0.93 & 0.96 \\
6 & 1.738 & 0.98 & 1.00 \\
5 & 1.534 & 0.97 & 0.98 \\
4 & 1.353 & 0.89 & 0.89 \\
3 & 1.194 & 1.01 & 1.03 \\
2 & 1.054 & 0.87 & 0.88 \\
1 & 0.930 & 0.97 & 0.97 \\
\hline
\end{tabular}

Figure 5.1-1: BUGLE 80 and BUGLE 96 data comparison. Detector at $10.16 \mathrm{~cm}$ from source.

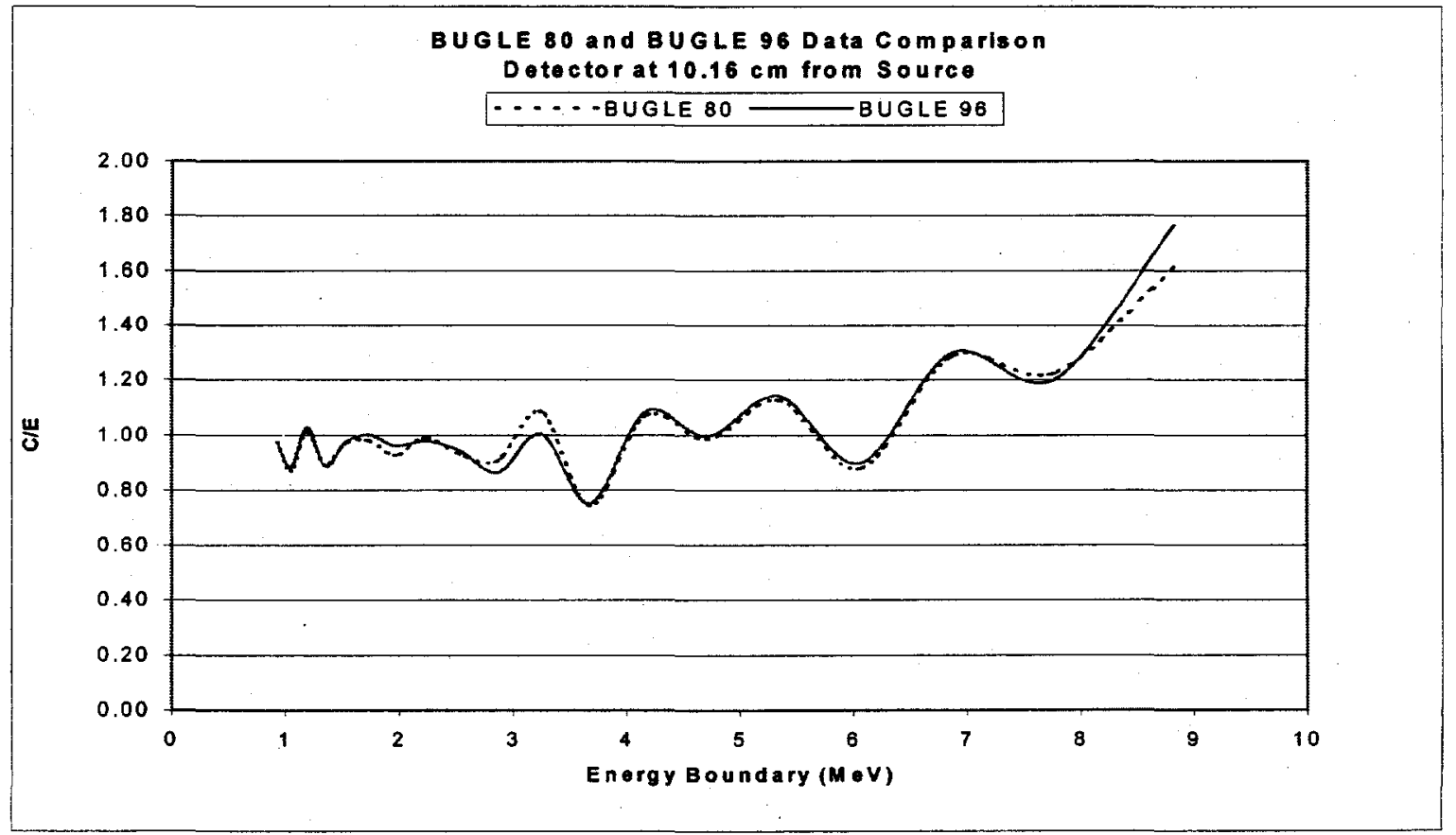


Table 5.1-4: BUGLE 80 results for a detector at a distance of $15.24 \mathrm{~cm}$ from source.

\begin{tabular}{|c|c|c|c|c|}
\hline \multirow[b]{2}{*}{ Group } & \multirow[b]{2}{*}{$\begin{array}{l}\text { Energy boundary } \\
(\mathrm{MeV})\end{array}$} & \multicolumn{2}{|c|}{ Flux/Lethargy per Source Neutron } & \multirow[b]{2}{*}{$\mathrm{C} / \mathrm{E}$} \\
\hline & & $\begin{array}{c}\text { Calculated (C) } \\
\left(\mathrm{cm}^{-2} / \mathrm{s}\right)\end{array}$ & $\begin{array}{c}\text { Experimental (E) } \\
\left(\mathrm{cm}^{-2} / \mathrm{s}\right)\end{array}$ & \\
\hline 20 & 10 & & & \\
\hline 19 & 8.825 & $3.646 \mathrm{E}-06$ & $2.67 \mathrm{E}-06$ & 1.37 \\
\hline 18 & 7.788 & $5.770 \mathrm{E}-06$ & $4.40 \mathrm{E}-06$ & 1.31 \\
\hline 17 & 6.873 & $9.752 \mathrm{E}-06$ & $8.33 \mathrm{E}-06$ & 1.17 \\
\hline 16 & 6.065 & $1.115 \mathrm{E}-05$ & $1.41 \mathrm{E}-05$ & 0.79 \\
\hline 15 & 5.353 & $2.013 \mathrm{E}-05$ & $1.80 \mathrm{E}-05$ & 1.12 \\
\hline 14 & 4.724 & $2.389 \mathrm{E}-05$ & $2.35 \mathrm{E}-05$ & 1.02 \\
\hline 13 & 4.169 & $2.972 \mathrm{E}-05$ & $3.06 \mathrm{E}-05$ & 0.97 \\
\hline 12 & 3.679 & $2.623 \mathrm{E}-05$ & $3.09 \mathrm{E}-05$ & 0.85 \\
\hline 11 & 3.247 & $3.299 \mathrm{E}-05$ & $3.40 \mathrm{E}-05$ & 0.97 \\
\hline 10 & 2.865 & $3.632 \mathrm{E}-05$ & $4.32 \mathrm{E}-05$ & 0.84 \\
\hline 9 & 2.528 & $4.620 \mathrm{E}-05$ & 4.83E-05 & 0.96 \\
\hline 8 & 2.231 & $5.396 \mathrm{E}-05$ & $5.41 \mathrm{E}-05$ & 1.00 \\
\hline 7 & 1.969 & $4.576 \mathrm{E}-05$ & $4.76 \mathrm{E}-05$ & 0.96 \\
\hline 6 & 1.738 & $4.464 \mathrm{E}-05$ & $4.45 \mathrm{E}-05$ & 1.00 \\
\hline 5 & 1.534 & $4.116 \mathrm{E}-05$ & $4.63 \mathrm{E}-05$ & 0.89 \\
\hline 4 & 1.353 & $3.680 \mathrm{E}-05$ & $4.52 \mathrm{E}-05$ & 0.81 \\
\hline 3 & 1.194 & $3.950 \mathrm{E}-05$ & $3.87 \mathrm{E}-05$ & 1.02 \\
\hline 2 & 1.054 & $3.487 \mathrm{E}-05$ & $4.27 \mathrm{E}-05$ & 0.82 \\
\hline 1 & 0.930 & $3.864 \mathrm{E}-05$ & $3.49 \mathrm{E}-05$ & 1.11 \\
\hline
\end{tabular}


BUGLE 96 VALIDATION WITH MORSE USING WATER

WSRC-TR-00349

AND IRON EXPERIMENTAL DATA FROM SINDBAD 97

Rev.0

Page 32 of 89

Table 5.1-5: BUGLE 96 results for a detector at a distance of $15.24 \mathrm{~cm}$ from source.

\begin{tabular}{|c|c|c|c|c|}
\hline \multirow{2}{*}{ Group } & $\begin{array}{c}\text { Energy boundary } \\
(\mathrm{MeV})\end{array}$ & $\begin{array}{c}\text { Flux/Lethargy per Source Neutron } \\
\left(\mathrm{cm}^{-2} / \mathrm{s}\right)\end{array}$ & $\begin{array}{c}\text { Experimental }(\mathrm{E}) \\
\left(\mathrm{cm}^{-2} / \mathrm{s}\right)\end{array}$ & $\mathrm{C} / \mathrm{E}$ \\
\hline 20 & 10 & & & \\
\hline 19 & 8.825 & $3.445 \mathrm{E}-06$ & $2.67 \mathrm{E}-06$ & 1.29 \\
\hline 18 & 7.788 & $5.563 \mathrm{E}-06$ & $4.40 \mathrm{E}-06$ & 1.26 \\
\hline 17 & 6.873 & $9.926 \mathrm{E}-06$ & $8.33 \mathrm{E}-06$ & 1.19 \\
\hline 16 & 6.065 & $1.153 \mathrm{E}-05$ & $1.41 \mathrm{E}-05$ & 0.82 \\
\hline 15 & 5.353 & $1.961 \mathrm{E}-05$ & $1.80 \mathrm{E}-05$ & 1.09 \\
\hline 14 & 4.724 & $2.353 \mathrm{E}-05$ & $2.35 \mathrm{E}-05$ & 1.00 \\
\hline 13 & 4.169 & $2.954 \mathrm{E}-05$ & $3.06 \mathrm{E}-05$ & 0.97 \\
\hline 12 & 3.679 & $2.607 \mathrm{E}-05$ & $3.09 \mathrm{E}-05$ & 0.84 \\
\hline 11 & 3.247 & $3.631 \mathrm{E}-05$ & $3.40 \mathrm{E}-05$ & 1.07 \\
\hline 10 & 2.865 & $3.707 \mathrm{E}-05$ & $4.32 \mathrm{E}-05$ & 0.86 \\
\hline 9 & 2.528 & $4.567 \mathrm{E}-05$ & $4.83 \mathrm{E}-05$ & 0.95 \\
\hline 8 & 2.231 & $5.991 \mathrm{E}-05$ & $5.41 \mathrm{E}-05$ & 1.11 \\
\hline 7 & 1.969 & $7.145 \mathrm{E}-05$ & $4.76 \mathrm{E}-05$ & 1.50 \\
\hline 6 & 1.738 & $5.183 \mathrm{E}-05$ & $4.45 \mathrm{E}-05$ & 1.16 \\
\hline 5 & 1.534 & $4.453 \mathrm{E}-05$ & $4.63 \mathrm{E}-05$ & 0.96 \\
\hline 4 & 1.353 & $4.016 \mathrm{E}-05$ & $4.52 \mathrm{E}-05$ & 0.89 \\
\hline 3 & 1.194 & $3.826 \mathrm{E}-05$ & $3.87 \mathrm{E}-05$ & 0.99 \\
\hline 2 & 1.054 & $3.377 \mathrm{E}-05$ & $4.27 \mathrm{E}-05$ & 0.79 \\
\hline 1 & 0.930 & $3.194 \mathrm{E}-05$ & $3.49 \mathrm{E}-05$ & 0.92 \\
\hline
\end{tabular}


Table 5.1-6: BUGLE 80 and BUGLE 96 comparison. Detector at $15.24 \mathrm{~cm}$ from source.

\begin{tabular}{|c|c|c|c|}
\hline Group & Energy Boundary & \multicolumn{2}{|c|}{ Calculated/Experimental } \\
\hline & $(\mathrm{MeV})$ & BUGLE 80 & BUGLE 96 \\
\hline 20 & 10 & & \\
19 & 8.825 & 1.37 & 1.29 \\
18 & 7.788 & 1.31 & 1.26 \\
17 & 6.873 & 1.17 & 1.19 \\
16 & 6.065 & 0.79 & 0.82 \\
15 & 5.353 & 1.12 & 1.09 \\
14 & 4.724 & 1.02 & 1.00 \\
13 & 4.169 & 0.97 & 0.97 \\
12 & 3.679 & 0.85 & 0.84 \\
11 & 3.247 & 0.97 & 1.07 \\
10 & 2.865 & 0.84 & 0.86 \\
9 & 2.528 & 0.96 & 0.95 \\
8 & 2.231 & 1.00 & 1.11 \\
7 & 1.969 & 0.96 & 1.50 \\
6 & 1.738 & 1.00 & 1.16 \\
5 & 1.534 & 0.89 & 0.96 \\
4 & 1.353 & 0.81 & 0.89 \\
3 & 1.194 & 1.02 & 0.99 \\
2 & 1.054 & 0.82 & 0.79 \\
1 & 0.930 & 1.11 & 0.92 \\
\hline
\end{tabular}

Figure 5.1-2: BUGLE 80 and BUGLE 96 data comparison. Detector at $15.24 \mathrm{~cm}$ from source.

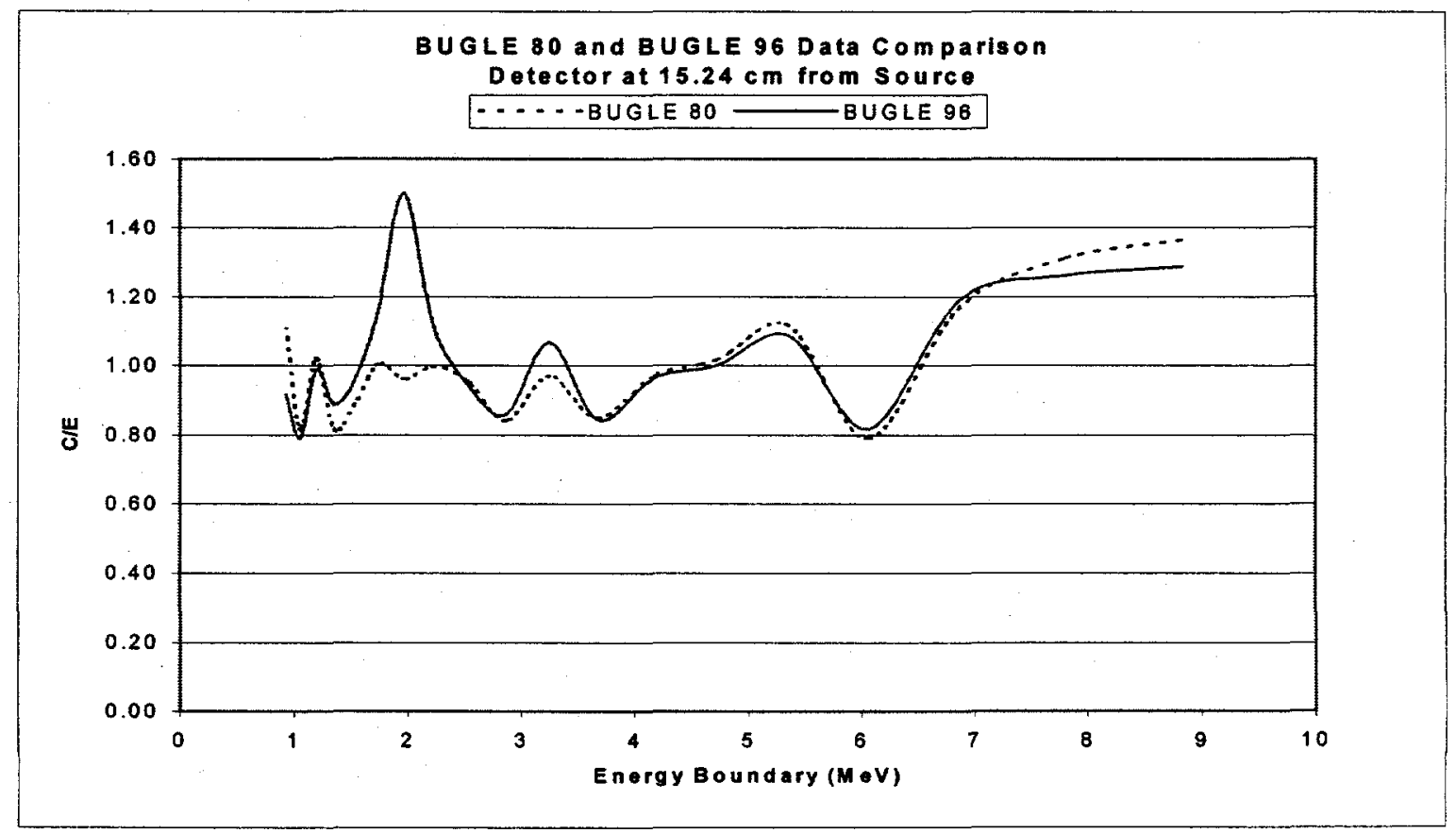


BUGLE 96 VALIDATION WITH MORSE USING WATER

WSRC-TR-00349

AND IRON EXPERIMENTAL DATA FROM SINDBAD 97

Rev.0

Page 34 of 89

Table 5.1-7: BUGLE 80 results for a detector at a distance of $20.32 \mathrm{~cm}$ from source.

\begin{tabular}{|c|c|c|c|c|}
\hline \multirow[b]{2}{*}{ Group } & \multirow[b]{2}{*}{$\begin{array}{c}\text { Energy boundary } \\
(\mathrm{MeV})\end{array}$} & \multicolumn{2}{|c|}{ Flux/Lethargy per Source Neutron } & \multirow[b]{2}{*}{$\mathrm{C} / \mathrm{E}$} \\
\hline & & $\begin{array}{c}\begin{array}{c}\text { Calculated (C) } \\
\left(\mathrm{cm}^{-2} / \mathrm{s}\right)\end{array} \\
\end{array}$ & $\begin{array}{c}\text { Experimental (E) } \\
\left(\mathrm{cm}^{-2} / \mathrm{s}\right)\end{array}$ & \\
\hline 20 & 10 & & & \\
\hline 19 & 8.825 & $1.176 \mathrm{E}-06$ & $1.04 \mathrm{E}-06$ & 1.13 \\
\hline 18 & 7.788 & $1.957 \mathrm{E}-06$ & $1.62 \mathrm{E}-06$ & 1.21 \\
\hline 17 & 6.873 & $3.694 \mathrm{E}-06$ & $2.96 \mathrm{E}-06$ & 1.25 \\
\hline 16 & 6.065 & $4.357 \mathrm{E}-06$ & $4.74 \mathrm{E}-06$ & 0.92 \\
\hline 15 & 5.353 & $6.597 \mathrm{E}-06$ & $6.07 \mathrm{E}-06$ & 1.09 \\
\hline 14 & 4.724 & $7.700 \mathrm{E}-06$ & $8.35 \mathrm{E}-06$ & 0.92 \\
\hline 13 & 4.169 & $9.445 \mathrm{E}-06$ & $9.14 \mathrm{E}-06$ & 1.03 \\
\hline 12 & 3.679 & $8.335 \mathrm{E}-06$ & $9.60 \mathrm{E}-06$ & 0.87 \\
\hline 11 & 3.247 & $1.016 \mathrm{E}-05$ & $9.20 \mathrm{E}-06$ & 1.10 \\
\hline 10 & 2.865 & $1.036 \mathrm{E}-05$ & $1.29 \mathrm{E}-05$ & 0.80 \\
\hline 9 & 2.528 & $1.326 \mathrm{E}-05$ & $1.43 \mathrm{E}-05$ & 0.93 \\
\hline 8 & 2.231 & $1.598 \mathrm{E}-05$ & $1.63 \mathrm{E}-05$ & 0.98 \\
\hline 7 & 1.969 & $1.331 \mathrm{E}-05$ & $1.42 \mathrm{E}-05$ & 0.94 \\
\hline 6 & 1.738 & $1.223 \mathrm{E}-05$ & $1.35 \mathrm{E}-05$ & 0.91 \\
\hline 5 & 1.534 & $1.216 \mathrm{E}-05$ & $1.28 \mathrm{E}-05$ & 0.95 \\
\hline 4 & 1.353 & $1.152 \mathrm{E}-05$ & $1.34 \mathrm{E}-05$ & 0.86 \\
\hline 3 & 1.194 & $9.938 \mathrm{E}-06$ & $1.15 \mathrm{E}-05$ & 0.86 \\
\hline 2 & 1.054 & $8.772 \mathrm{E}-06$ & $1.30 \mathrm{E}-05$ & 0.67 \\
\hline 1 & 0.930 & $8.054 \mathrm{E}-06$ & $1.07 \mathrm{E}-05$ & 0.75 \\
\hline
\end{tabular}


Table 5.1-8: BUGLE 96 results for a detector at a distance of $20.32 \mathrm{~cm}$ from source.

\begin{tabular}{|c|c|c|c|c|}
\hline \multirow[b]{2}{*}{ Group } & \multirow[b]{2}{*}{$\begin{array}{c}\text { Energy boundary } \\
(\mathrm{MeV})\end{array}$} & \multicolumn{2}{|c|}{ Flux/Lethargy per Source Neutron } & \multirow[b]{2}{*}{$\mathrm{C} / \mathrm{E}$} \\
\hline & & \begin{tabular}{|c}
$\begin{array}{c}\text { Calculated (C) } \\
\left(\mathrm{cm}^{-2} / \mathrm{s}\right)\end{array}$ \\
\end{tabular} & $\begin{array}{c}\text { Experimental (E) } \\
\left(\mathrm{cm}^{-2} / \mathrm{s}\right)\end{array}$ & \\
\hline 20 & 10 & & & \\
\hline 19 & 8.825 & $1.136 \mathrm{E}-06$ & $1.04 \mathrm{E}-06$ & 1.09 \\
\hline 18 & 7.788 & $1.954 \mathrm{E}-06$ & $1.62 \mathrm{E}-06$ & 1.21 \\
\hline 17 & 6.873 & $3.086 \mathrm{E}-06$ & $2.96 \mathrm{E}-06$ & 1.04 \\
\hline 16 & 6.065 & $3.436 \mathrm{E}-06$ & $4.74 \mathrm{E}-06$ & 0.72 \\
\hline 15 & 5.353 & $6.295 \mathrm{E}-06$ & $6.07 \mathrm{E}-06$ & 1.04 \\
\hline 14 & 4.724 & $7.598 \mathrm{E}-06$ & $8.35 \mathrm{E}-06$ & 0.91 \\
\hline 13 & 4.169 & $9.588 \mathrm{E}-06$ & $9.14 \mathrm{E}-06$ & 1.05 \\
\hline 12 & 3.679 & $8.461 \mathrm{E}-06$ & $9.60 \mathrm{E}-06$ & 0.88 \\
\hline 11 & 3.247 & $1.559 \mathrm{E}-05$ & $9.20 \mathrm{E}-06$ & 1.69 \\
\hline 10 & 2.865 & $1.345 \mathrm{E}-05$ & $1.29 \mathrm{E}-05$ & 1.04 \\
\hline 9 & 2.528 & $1.311 \mathrm{E}-05$ & $1.43 \mathrm{E}-05$ & 0.92 \\
\hline 8 & 2.231 & $1.499 \mathrm{E}-05$ & $1.63 \mathrm{E}-05$ & 0.92 \\
\hline 7 & 1.969 & $1.242 \mathrm{E}-05$ & $1.42 \mathrm{E}-05$ & 0.87 \\
\hline 6 & 1.738 & $1.234 \mathrm{E}-05$ & $1.35 \mathrm{E}-05$ & 0.91 \\
\hline 5 & 1.534 & $1.204 \mathrm{E}-05$ & $1.28 \mathrm{E}-05$ & 0.94 \\
\hline 4 & 1.353 & $1.115 \mathrm{E}-05$ & $1.34 \mathrm{E}-05$ & 0.83 \\
\hline 3 & 1.194 & $9.236 \mathrm{E}-06$ & $1.15 \mathrm{E}-05$ & 0.80 \\
\hline 2 & 1.054 & $8.152 \mathrm{E}-06$ & $1.30 \mathrm{E}-05$ & 0.63 \\
\hline 1 & 0.930 & $8.081 \mathrm{E}-06$ & $1.07 \mathrm{E}-05$ & 0.76 \\
\hline
\end{tabular}


BUGLE 96 VALIDATION WITH MORSE USING WATER

WSRC-TR-00349 AND IRON EXPERIMENTAL DATA FROM SINDBAD 97

Rev.0

Page 36 of 89

Table 5.1-9: BUGLE 80 and BUGLE 96 comparison. Detector at $20.32 \mathrm{~cm}$ from source.

\begin{tabular}{|c|c|c|c|}
\hline Group & Energy Boundary & \multicolumn{2}{|c|}{ Calculated/Experimental } \\
\hline & $(\mathrm{MeV})$ & BUGLE 80 & BUGLE 96 \\
\hline 20 & 10 & 1.13 & 1.09 \\
19 & 8.825 & 1.21 & 1.21 \\
18 & 7.788 & 1.25 & 1.04 \\
17 & 6.873 & 0.92 & 0.72 \\
16 & 6.065 & 1.09 & 1.04 \\
15 & 5.353 & 0.92 & 0.91 \\
14 & 4.724 & 1.03 & 1.05 \\
13 & 4.169 & 0.87 & 0.88 \\
12 & 3.679 & 1.10 & 1.69 \\
11 & 3.247 & 0.80 & 1.04 \\
10 & 2.865 & 0.93 & 0.92 \\
9 & 2.528 & 0.98 & 0.92 \\
8 & 2.231 & 0.94 & 0.87 \\
7 & 1.969 & 0.91 & 0.91 \\
6 & 1.738 & 0.95 & 0.94 \\
5 & 1.534 & 0.86 & 0.83 \\
4 & 1.353 & 0.86 & 0.80 \\
3 & 1.194 & 0.67 & 0.63 \\
2 & 1.054 & 0.75 & 0.76 \\
1 & 0.930 & & \\
\hline
\end{tabular}

Figure 5.1-3: BUGLE 80 and BUGLE 96 data comparison. Detector at $20.32 \mathrm{~cm}$ from source.

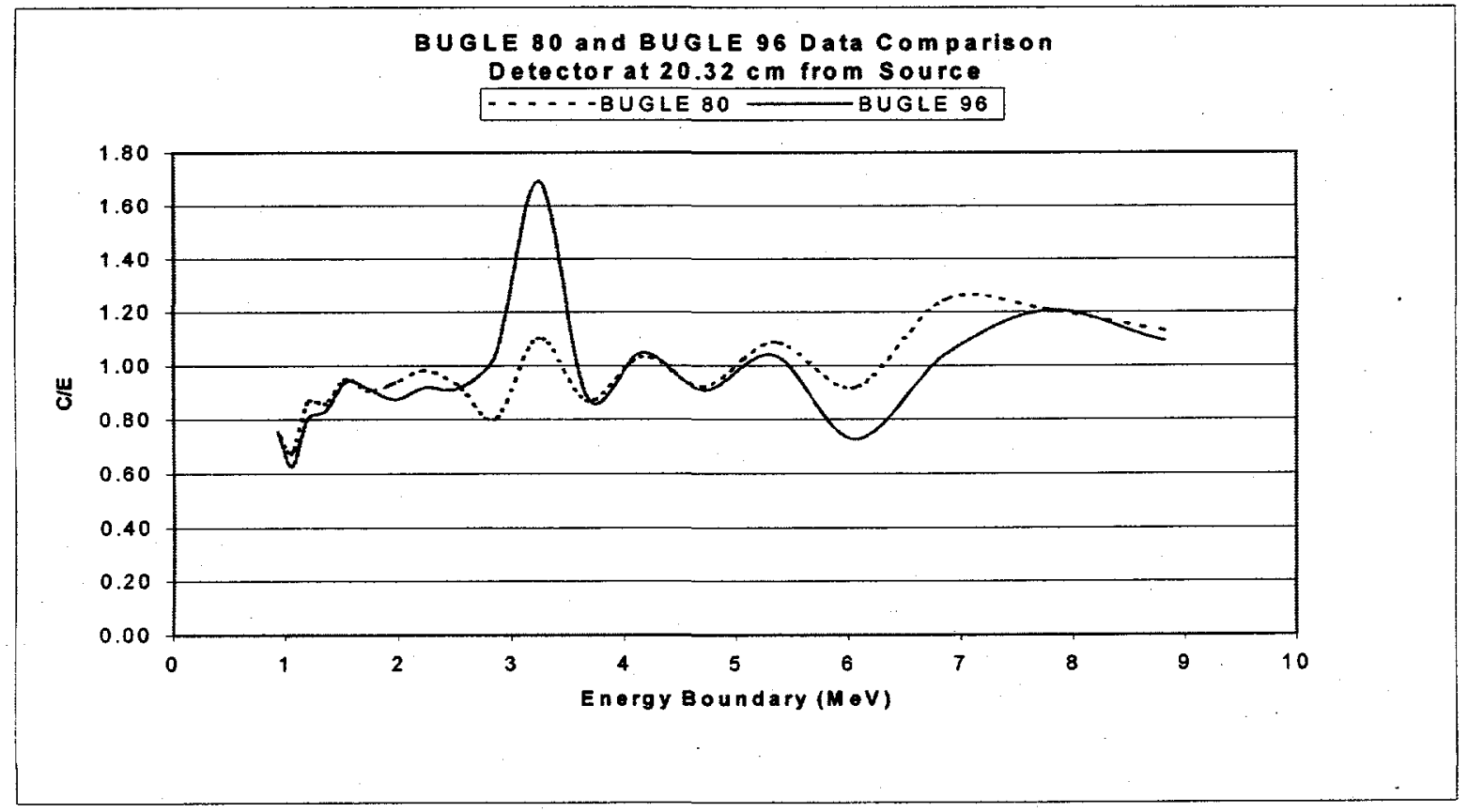


Table 5.1-10: BUGLE 80 results for a detector at a distance of $25.40 \mathrm{~cm}$ from source.

\begin{tabular}{|c|c|c|c|c|}
\hline \multirow[b]{2}{*}{ Group } & \multirow[b]{2}{*}{$\begin{array}{l}\text { Energy boundary } \\
(\mathrm{MeV})\end{array}$} & \multicolumn{2}{|c|}{ Flux/Lethargy per Source Neutron } & \multirow[b]{2}{*}{$\mathrm{C} / \mathrm{E}$} \\
\hline & & $\begin{array}{c}\text { Calculated (C) } \\
\left(\mathrm{cm}^{-2} / \mathrm{s}\right)\end{array}$ & $\begin{array}{c}\text { Experimental (E) } \\
\left(\mathrm{cm}^{-2} / \mathrm{s}\right)\end{array}$ & \\
\hline 20 & 10 & & & \\
\hline 19 & 8.825 & $5.260 \mathrm{E}-07$ & 3.74E-07 & 1.41 \\
\hline 18 & 7.788 & $7.106 \mathrm{E}-07$ & $7.15 \mathrm{E}-07$ & 0.99 \\
\hline 17 & 6.873 & $1.514 \mathrm{E}-06$ & $1.14 \mathrm{E}-06$ & 1.33 \\
\hline 16 & 6.065 & $1.855 \mathrm{E}-06$ & $1.83 \mathrm{E}-06$ & 1.01 \\
\hline 15 & 5.353 & $2.677 \mathrm{E}-06$ & $2.44 \mathrm{E}-06$ & 1.10 \\
\hline 14 & 4.724 & $2.851 \mathrm{E}-06$ & $3.03 \mathrm{E}-06$ & 0.94 \\
\hline 13 & 4.169 & $3.206 \mathrm{E}-06$ & $3.38 \mathrm{E}-06$ & 0.95 \\
\hline 12 & 3.679 & $2.829 \mathrm{E}-06$ & $3.46 \mathrm{E}-06$ & 0.82 \\
\hline 11 & 3.247 & $3.486 \mathrm{E}-06$ & $3.27 \mathrm{E}-06$ & 1.07 \\
\hline 10 & 2.865 & $3.643 \mathrm{E}-06$ & $4.24 \mathrm{E}-06$ & 0.86 \\
\hline 9 & 2.528 & $4.699 \mathrm{E}-06$ & $5.06 \mathrm{E}-06$ & 0.93 \\
\hline 8 & 2.231 & $4.835 \mathrm{E}-06$ & $5.53 \mathrm{E}-06$ & 0.87 \\
\hline 7 & 1.969 & $4.002 \mathrm{E}-06$ & $4.96 \mathrm{E}-06$ & 0.81 \\
\hline 6 & 1.738 & $3.661 \mathrm{E}-06$ & $4.51 \mathrm{E}-06$ & 0.81 \\
\hline 5 & 1.534 & $3.400 \mathrm{E}-06$ & $4.53 \mathrm{E}-06$ & 0.75 \\
\hline 4 & 1.353 & $3.088 \mathrm{E}-06$ & $4.55 \mathrm{E}-06$ & 0.68 \\
\hline 3 & 1.194 & $3.178 \mathrm{E}-06$ & 4.19E-06 & 0.76 \\
\hline 2 & 1.054 & $2.805 \mathrm{E}-06$ & 4.97E-06 & 0.56 \\
\hline 1 & 0.930 & $2.607 \mathrm{E}-06$ & $4.07 \mathrm{E}-06$ & 0.64 \\
\hline
\end{tabular}


Table 5.1-11: BUGLE 96 results for a detector at a distance of $25.40 \mathrm{~cm}$ from source.

\begin{tabular}{|c|c|c|c|c|}
\hline \multirow[b]{2}{*}{ Group } & \multirow[b]{2}{*}{$\begin{array}{c}\text { Energy boundary } \\
(\mathrm{MeV})\end{array}$} & \multicolumn{2}{|c|}{ Flux/Lethargy per Source Neutron } & \multirow[b]{2}{*}{$\mathrm{C} / \mathrm{E}$} \\
\hline & & $\begin{array}{c}\text { Calculated (C) } \\
\left(\mathrm{cm}^{-2} / \mathrm{s}\right)\end{array}$ & $\begin{array}{c}\text { Experimental (E) } \\
\left(\mathrm{cm}^{-2} / \mathrm{s}\right)\end{array}$ & \\
\hline 20 & 10 & & & \\
\hline 19 & 8.825 & $4.772 \mathrm{E}-07$ & $3.74 \mathrm{E}-07$ & 1.28 \\
\hline 18 & 7.788 & $7.444 \mathrm{E}-07$ & $7.15 \mathrm{E}-07$ & 1.04 \\
\hline 17 & 6.873 & $1.642 \mathrm{E}-06$ & $1.14 \mathrm{E}-06$ & 1.44 \\
\hline 16 & 6.065 & $2.020 \mathrm{E}-06$ & $1.83 \mathrm{E}-06$ & 1.10 \\
\hline 15 & 5.353 & $2.517 \mathrm{E}-06$ & $2.44 \mathrm{E}-06$ & 1.03 \\
\hline 14 & 4.724 & $2.712 \mathrm{E}-06$ & $3.03 \mathrm{E}-06$ & 0.90 \\
\hline 13 & 4.169 & $3.087 \mathrm{E}-06$ & $3.38 \mathrm{E}-06$ & 0.91 \\
\hline 12 & 3.679 & $2.724 \mathrm{E}-06$ & $3.46 \mathrm{E}-06$ & 0.79 \\
\hline 11 & 3.247 & $4.223 \mathrm{E}-06$ & $3.27 \mathrm{E}-06$ & 1.29 \\
\hline 10 & 2.865 & $4.136 \mathrm{E}-06$ & $4.24 \mathrm{E}-06$ & 0.98 \\
\hline 9 & 2.528 & $4.220 \mathrm{E}-06$ & $5.06 \mathrm{E}-06$ & 0.83 \\
\hline 8 & 2.231 & $6.185 \mathrm{E}-06$ & $5.53 \mathrm{E}-06$ & 1.12 \\
\hline 7 & 1.969 & $4.115 \mathrm{E}-06$ & $4.96 \mathrm{E}-06$ & 0.83 \\
\hline 6 & 1.738 & $3.891 \mathrm{E}-06$ & $4.51 \mathrm{E}-06$ & 0.86 \\
\hline 5 & 1.534 & $3.899 \mathrm{E}-06$ & $4.53 \mathrm{E}-06$ & 0.86 \\
\hline 4 & 1.353 & $3.695 \mathrm{E}-06$ & $4.55 \mathrm{E}-06$ & 0.81 \\
\hline 3 & 1.194 & $377 \mathrm{E}-06$ & $4.19 \mathrm{E}-06$ & 0.83 \\
\hline 2 & 1.054 & -06 & $4.97 \mathrm{E}-06$ & 0.62 \\
\hline 1 & 0.930 & .06 & $4.07 \mathrm{E}-06$ & 0.66 \\
\hline
\end{tabular}


BUGLE 96 VALIDATION WITH MORSE USING WATER

WSRC-TR-00349

AND IRON EXPERIMENTAL DATA FROM SINDBAD 97

Rev.0

Page 39 of 89

Table 5.1-12: BUGLE 80 and BUGLE 96 comparison. Detector at $25.40 \mathrm{~cm}$ from source.

\begin{tabular}{|c|c|c|c|}
\hline Group & Energy Boundary & \multicolumn{2}{|c|}{ Calculated/Experimental } \\
\hline & $(\mathrm{MeV})$ & BUGLE 80 & BUGLE 96 \\
\hline 20 & 10 & 1.41 & 1.28 \\
19 & 8.825 & 0.99 & 1.04 \\
18 & 7.788 & 1.33 & 1.44 \\
17 & 6.873 & 1.01 & 1.10 \\
16 & 6.065 & 1.10 & 1.03 \\
15 & 5.353 & 0.94 & 0.90 \\
14 & 4.724 & 0.95 & 0.91 \\
13 & 4.169 & 0.82 & 0.79 \\
12 & 3.679 & 1.07 & 1.29 \\
11 & 3.247 & 0.86 & 0.98 \\
10 & 2.865 & 0.93 & 0.83 \\
9 & 2.528 & 0.87 & 1.12 \\
8 & 2.231 & 0.81 & 0.83 \\
7 & 1.969 & 0.81 & 0.86 \\
6 & 1.738 & 0.75 & 0.86 \\
5 & 1.534 & 0.68 & 0.81 \\
4 & 1.353 & 0.76 & 0.83 \\
3 & 1.194 & 0.56 & 0.62 \\
2 & 1.054 & 0.64 & 0.66 \\
1 & 0.930 & &
\end{tabular}

Figure 5.1-4: BUGLE 80 and BUGLE 96 data comparison. Detector at $25.40 \mathrm{~cm}$ from source.

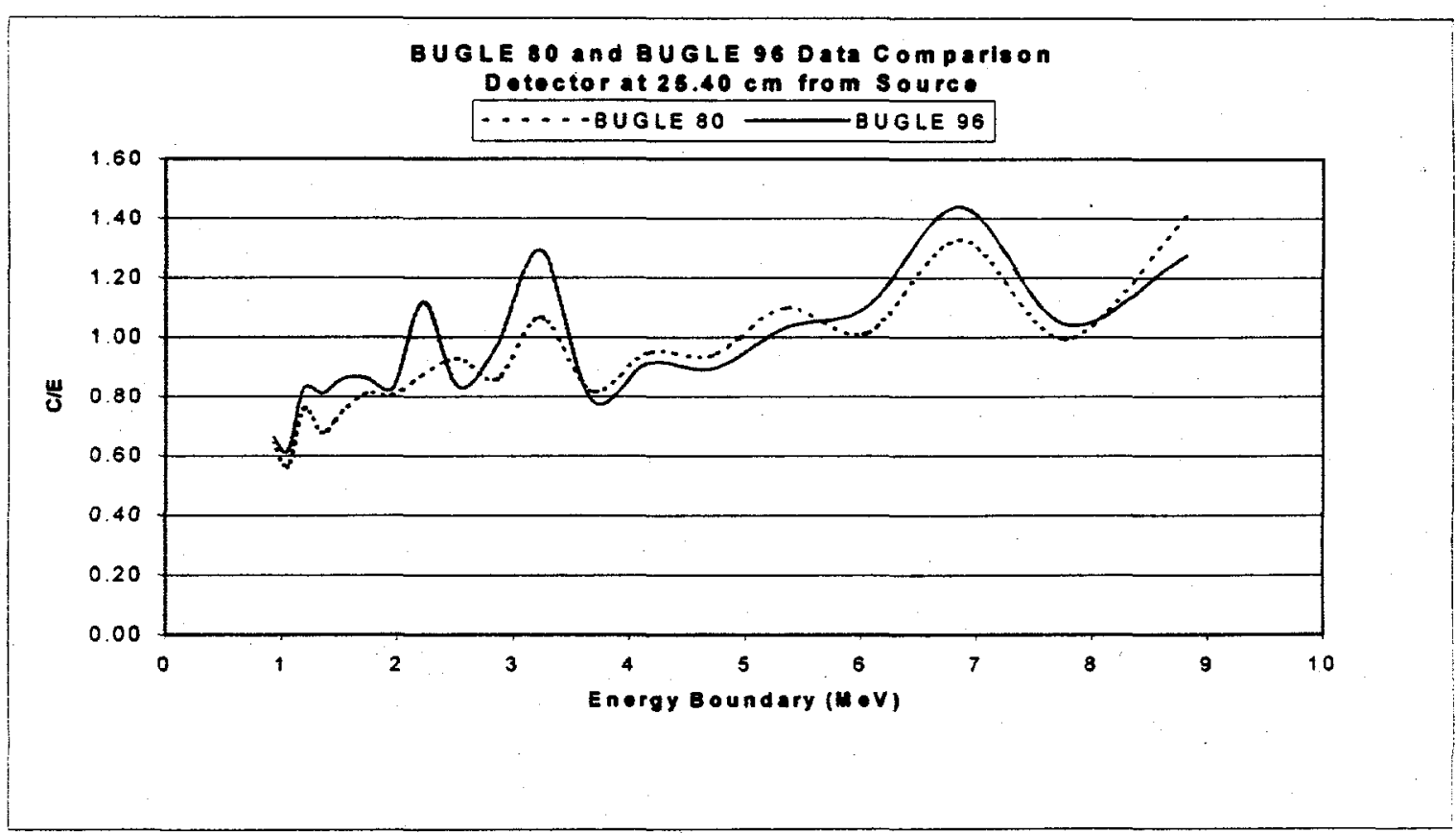


Table 5.1-13: BUGLE 80 results for a detector at a distance of $30.48 \mathrm{~cm}$ from source.

\begin{tabular}{|c|c|c|c|c|}
\hline \multirow[b]{2}{*}{ Group } & \multirow[b]{2}{*}{$\begin{array}{c}\text { Energy boundary } \\
(\mathrm{MeV})\end{array}$} & \multicolumn{2}{|c|}{ Flux/Lethargy per Source Neutron } & \multirow[b]{2}{*}{$\mathrm{C} / \mathrm{E}$} \\
\hline & & $\begin{array}{c}\text { Calculated (C) } \\
\left(\mathrm{cm}^{-2} / \mathrm{s}\right)\end{array}$ & $\begin{array}{c}\text { Experimental (E) } \\
\left(\mathrm{cm}^{-2} / \mathrm{s}\right)\end{array}$ & \\
\hline 20 & 10 & & & \\
\hline 19 & 8.825 & $2.394 \mathrm{E}-07$ & $1.77 \mathrm{E}-07$ & 1.35 \\
\hline 18 & 7.788 & $3.369 \mathrm{E}-07$ & $3.05 \mathrm{E}-07$ & 1.10 \\
\hline 17 & 6.873 & $6.079 \mathrm{E}-07$ & $5.46 \mathrm{E}-07$ & 1.11 \\
\hline 16 & 6.065 & $7.126 \mathrm{E}-07$ & $8.04 \mathrm{E}-07$ & 0.89 \\
\hline 15 & 5.353 & $9.009 \mathrm{E}-07$ & $9.77 \mathrm{E}-07$ & 0.92 \\
\hline 14 & 4.724 & $1.077 \mathrm{E}-06$ & $1.24 \mathrm{E}-06$ & 0.87 \\
\hline 13 & 4.169 & $1.348 \mathrm{E}-06$ & $1.36 \mathrm{E}-06$ & 0.99 \\
\hline 12 & 3.679 & $1.189 \mathrm{E}-06$ & $1.26 \mathrm{E}-06$ & 0.94 \\
\hline 11 & 3.247 & $1.179 \mathrm{E}-06$ & $1.20 \mathrm{E}-06$ & 0.98 \\
\hline 10 & 2.865 & $1.234 \mathrm{E}-06$ & $1.58 \mathrm{E}-06$ & 0.78 \\
\hline 9 & 2.528 & $1.753 \mathrm{E}-06$ & $1.81 \mathrm{E}-06$ & 0.97 \\
\hline 8 & 2.231 & $1.844 \mathrm{E}-06$ & $2.03 \mathrm{E}-06$ & $\overline{0.91}$ \\
\hline 7 & 1.969 & $1.279 \mathrm{E}-06$ & $1.75 \mathrm{E}-06$ & 0.73 \\
\hline 6 & 1.738 & $1.430 \mathrm{E}-06$ & $1.62 \mathrm{E}-06$ & 0.88 \\
\hline 5 & 1.534 & $1.156 \mathrm{E}-06$ & $1.57 \mathrm{E}-06$ & 0.74 \\
\hline 4 & 1.353 & $9.092 \mathrm{E}-07$ & $1.59 \mathrm{E}-06$ & 0.57 \\
\hline 3 & 1.194 & $1.417 \mathrm{E}-06$ & $1.45 \mathrm{E}-06$ & $\overline{0.98}$ \\
\hline 2 & 1.054 & $1.251 \mathrm{E}-06$ & $1.75 \mathrm{E}-06$ & 0.71 \\
\hline 1 & 0.930 & $9.837 \mathrm{E}-07$ & $1.61 \mathrm{E}-06$ & 0.61 \\
\hline
\end{tabular}


BUGLE 96 VALIDATION WITH MORSE USING WATER

WSRC-TR-00349

AND IRON EXPERIMENTAL DATA FROM SINDBAD 97

Rev.0

Page 41 of 89

Table 5.1-14: BUGLE 96 results for a detector at a distance of $30.48 \mathrm{~cm}$ from source.

\begin{tabular}{|c|c|c|c|c|}
\hline \multirow[b]{2}{*}{ Group } & \multirow[b]{2}{*}{$\begin{array}{c}\text { Energy boundary } \\
(\mathrm{MeV})\end{array}$} & \multicolumn{2}{|c|}{ Flux/Lethargy per Source Neutron } & \multirow[b]{2}{*}{$\mathrm{C} / \mathrm{E}$} \\
\hline & & $\begin{array}{c}\text { Calculated (C) } \\
\left(\mathrm{cm}^{-2} / \mathrm{s}\right)\end{array}$ & $\begin{array}{c}\text { Experimental (E) } \\
\left(\mathrm{cm}^{-2} / \mathrm{s}\right)\end{array}$ & \\
\hline 20 & 10 & & & \\
\hline 19 & 8.825 & $2.033 \mathrm{E}-07$ & $1.77 \mathrm{E}-07$ & 1.15 \\
\hline 18 & 7.788 & $3.152 \mathrm{E}-07$ & $3.05 \mathrm{E}-07$ & 1.03 \\
\hline 17 & 6.873 & $6.078 \mathrm{E}-07$ & $5.46 \mathrm{E}-07$ & 1.11 \\
\hline 16 & 6.065 & $7.229 \mathrm{E}-07$ & 8.04E-07 & 0.90 \\
\hline 15 & 5.353 & $9.619 \mathrm{E}-07$ & $9.77 \mathrm{E}-07$ & 0.98 \\
\hline 14 & 4.724 & $1.092 \mathrm{E}-06$ & $1.24 \mathrm{E}-06$ & 0.88 \\
\hline 13 & 4.169 & $1.306 \mathrm{E}-06$ & $1.36 \mathrm{E}-06$ & 0.96 \\
\hline 12 & 3.679 & $1.153 \mathrm{E}-06$ & $1.26 \mathrm{E}-06$ & 0.91 \\
\hline 11 & 3.247 & $1.395 \mathrm{E}-06$ & $1.20 \mathrm{E}-06$ & 1.16 \\
\hline 10 & 2.865 & $1.430 \mathrm{E}-06$ & $1.58 \mathrm{E}-06$ & 0.91 \\
\hline 9 & 2.528 & $1.541 \mathrm{E}-06$ & $1.81 \mathrm{E}-06$ & 0.85 \\
\hline 8 & 2.231 & $1.650 \mathrm{E}-06$ & $2.03 \mathrm{E}-06$ & 0.81 \\
\hline 7 & 1.969 & $1.435 \mathrm{E}-06$ & $1.75 \mathrm{E}-06$ & 0.82 \\
\hline 6 & 1.738 & $1.350 \mathrm{E}-06$ & $1.62 \mathrm{E}-06$ & 0.83 \\
\hline 5 & 1.534 & $1.378 \mathrm{E}-06$ & $1.57 \mathrm{E}-06$ & 0.88 \\
\hline 4 & 1.353 & $1.321 \mathrm{E}-06$ & $1.59 \mathrm{E}-06$ & 0.83 \\
\hline 3 & 1.194 & $1.048 \mathrm{E}-06$ & $1.45 \mathrm{E}-06$ & 0.72 \\
\hline 2 & 1.054 & $9.248 \mathrm{E}-07$ & $1.75 \mathrm{E}-06$ & 0.53 \\
\hline 1 & 0.930 & $1.033 \mathrm{E}-06$ & $1.61 \mathrm{E}-06$ & 0.64 \\
\hline
\end{tabular}


Table 5.1-15: BUGLE 80 and BUGLE 96 comparison. Detector at $30.48 \mathrm{~cm}$ from source.

\begin{tabular}{|c|c|c|c|}
\hline Group & Energy Boundary & \multicolumn{2}{|c|}{ Calculated/Experimental } \\
\hline & $(\mathrm{MeV})$ & BUGLE 80 & BUGLE 96 \\
\hline 20 & 10 & & \\
19 & 8.825 & 1.35 & 1.15 \\
18 & 7.788 & 1.10 & 1.03 \\
17 & 6.873 & 1.11 & 1.11 \\
16 & 6.065 & 0.89 & 0.90 \\
15 & 5.353 & 0.92 & 0.98 \\
14 & 4.724 & 0.87 & 0.88 \\
13 & 4.169 & 0.99 & 0.96 \\
12 & 3.679 & 0.94 & 0.91 \\
11 & 3.247 & 0.98 & 1.16 \\
10 & 2.865 & 0.78 & 0.91 \\
9 & 2.528 & 0.97 & 0.85 \\
8 & 2.231 & 0.91 & 0.81 \\
7 & 1.969 & 0.73 & 0.82 \\
6 & 1.738 & 0.88 & 0.83 \\
5 & 1.534 & 0.74 & 0.88 \\
4 & 1.353 & 0.57 & 0.83 \\
3 & 1.194 & 0.98 & 0.72 \\
2 & 1.054 & 0.71 & 0.53 \\
1 & 0.930 & 0.61 & 0.64 \\
\hline
\end{tabular}

Figure 5.1-5: BUGLE 80 and BUGLE 96 data comparison. Detector at $30.48 \mathrm{~cm}$ from source.

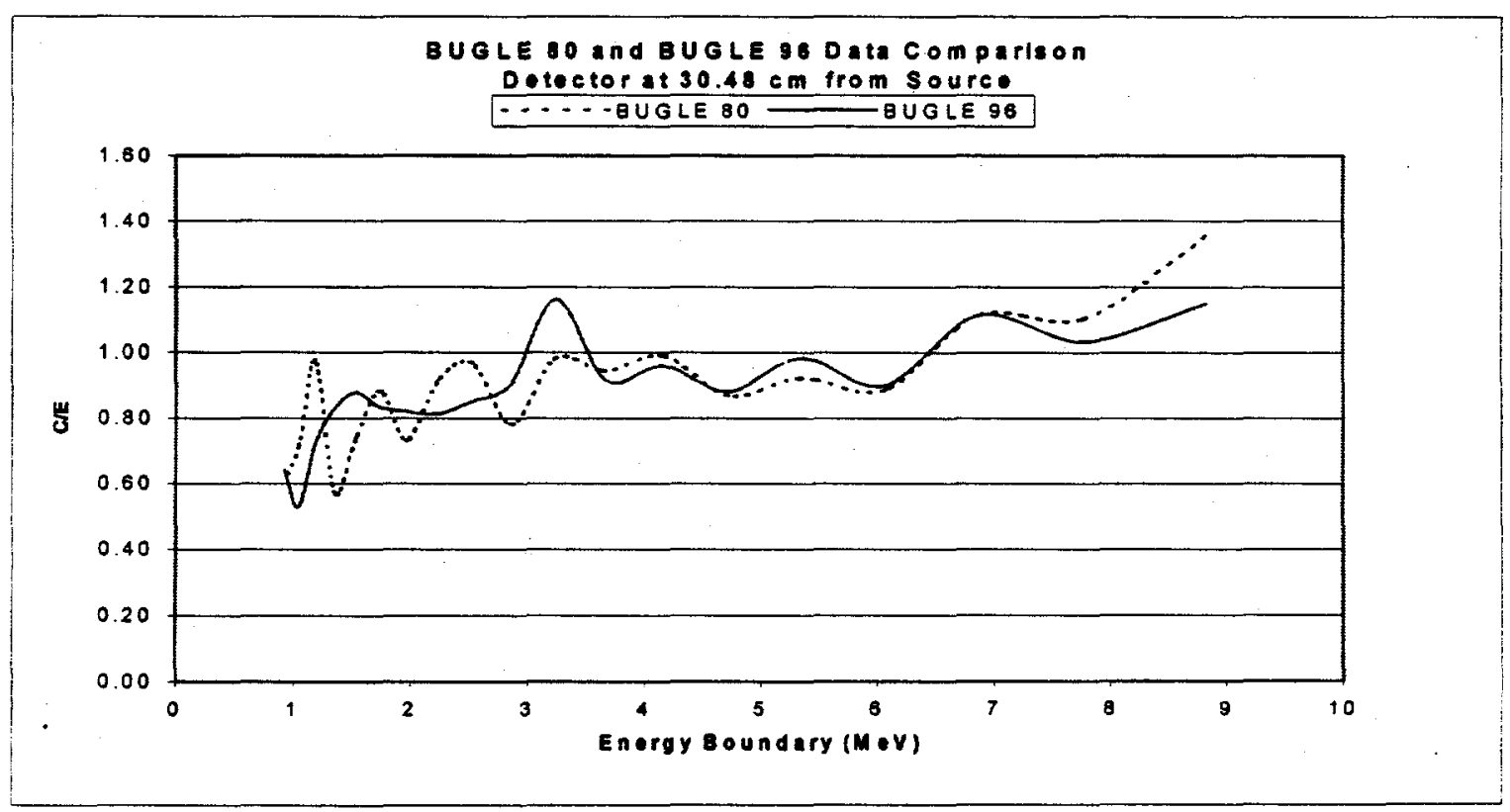


BUGLE 96 VALIDATION WITH MORSE USING WATER

WSRC-TR-00349

AND IRON EXPERIMENTAL DATA FROM SINDBAD 97

Rev.0

Page 43 of 89

Table 5.1-16: BUGLE 80 results for a detector at a distance of $35.56 \mathrm{~cm}$ from source.

\begin{tabular}{|c|c|c|c|c|}
\hline \multirow[b]{2}{*}{ Group } & \multirow[b]{2}{*}{$\begin{array}{c}\text { Energy boundary } \\
(\mathrm{MeV})\end{array}$} & \multicolumn{2}{|c|}{ Flux/Lethargy per Source Neutron } & \multirow[b]{2}{*}{$\mathrm{C} / \mathrm{E}$} \\
\hline & & $\begin{array}{c}\text { Calculated (C) } \\
\left(\mathrm{cm}^{-2} / \mathrm{s}\right)\end{array}$ & $\begin{array}{c}\text { Experimental (E) } \\
\left(\mathrm{cm}^{-2} / \mathrm{s}\right)\end{array}$ & \\
\hline 20 & 10 & & & \\
\hline 19 & 8.825 & $9.739 \mathrm{E}-08$ & $9.17 \mathrm{E}-08$ & 1.06 \\
\hline 18 & 7.788 & $1.502 \mathrm{E}-07$ & $1.38 \mathrm{E}-07$ & 1.09 \\
\hline 17 & 6.873 & $3.202 \mathrm{E}-07$ & $2.36 \mathrm{E}-07$ & 1.36 \\
\hline 16 & 6.065 & $3.907 \mathrm{E}-07$ & $3.42 \mathrm{E}-07$ & 1.14 \\
\hline 15 & 5.353 & $4.938 \mathrm{E}-07$ & $4.06 \mathrm{E}-07$ & 1.22 \\
\hline 14 & 4.724 & $5.637 \mathrm{E}-07$ & $5.34 \mathrm{E}-07$ & 1.06 \\
\hline 13 & 4.169 & $6.781 \mathrm{E}-07$ & $5.41 \mathrm{E}-07$ & 1.25 \\
\hline 12 & 3.679 & $5.984 \mathrm{E}-07$ & $5.13 \mathrm{E}-07$ & 1.17 \\
\hline 11 & 3.247 & $5.053 \mathrm{E}-07$ & $4.26 \mathrm{E}-07$ & 1.19 \\
\hline 10 & 2.865 & $4.966 \mathrm{E}-07$ & $6.08 \mathrm{E}-07$ & 0.82 \\
\hline 9 & 2.528 & $5.641 \mathrm{E}-07$ & $6.60 \mathrm{E}-07$ & 0.85 \\
\hline 8 & 2.231 & $6.561 \mathrm{E}-07$ & $7.58 \mathrm{E}-07$ & 0.87 \\
\hline 7 & 1.969 & $8.960 \mathrm{E}-07$ & $6.43 \mathrm{E}-07$ & 1.39 \\
\hline 6 & 1.738 & $5.776 \mathrm{E}-07$ & $5.96 \mathrm{E}-07$ & 0.97 \\
\hline 5 & 1.534 & $4.962 \mathrm{E}-07$ & $5.72 \mathrm{E}-07$ & 0.87 \\
\hline$\overline{4}$ & 1.353 & $4.603 \mathrm{E}-07$ & $5.71 \mathrm{E}-07$ & 0.81 \\
\hline 3 & 1.194 & $4.762 \mathrm{E}-07$ & $5.18 \mathrm{E}-07$ & 0.92 \\
\hline 2 & 1.054 & $4.203 \mathrm{E}-07$ & $6.19 \mathrm{E}-07$ & 0.68 \\
\hline 1 & 0.930 & $3.494 \mathrm{E}-07$ & $5.38 \mathrm{E}-07$ & 0.65 \\
\hline
\end{tabular}


BUGLE 96 VALIDATION WITH MORSE USING WATER

WSRC-TR-00349

AND IRON EXPERIMENTAL DATA FROM SINDBAD 97

Rev.0

Page 44 of 89

Table 5.1-17: BUGLE 96 results for a detector at a distance of $35.56 \mathrm{~cm}$ from source.

\begin{tabular}{|c|c|c|c|c|}
\hline \multirow[b]{2}{*}{ Group } & \multirow[b]{2}{*}{$\begin{array}{c}\text { Energy boundary } \\
(\mathrm{MeV})\end{array}$} & \multicolumn{2}{|c|}{ Flux/Lethargy per Source Neutron } & \multirow[b]{2}{*}{$\mathrm{C} / \mathrm{E}$} \\
\hline & & $\begin{array}{c}\text { Calculated (C) } \\
\left(\mathrm{cm}^{-2} / \mathrm{s}\right)\end{array}$ & $\begin{array}{c}\text { Experimental }(E) \\
\left(\mathrm{cm}^{-2} / \mathrm{s}\right)\end{array}$ & \\
\hline 20 & 10 & & & \\
\hline 19 & 8.825 & $1.098 \mathrm{E}-07$ & $9.17 \mathrm{E}-08$ & 1.20 \\
\hline 18 & 7.788 & $1.542 \mathrm{E}-07$ & $1.38 \mathrm{E}-07$ & 1.12 \\
\hline 17 & 6.873 & $2.591 \mathrm{E}-07$ & $2.36 \mathrm{E}-07$ & 1.10 \\
\hline 16 & 6.065 & $2.973 \mathrm{E}-07$ & $3.42 \mathrm{E}-07$ & 0.87 \\
\hline 15 & 5.353 & $4.116 \mathrm{E}-07$ & $4.06 \mathrm{E}-07$ & 1.01 \\
\hline 14 & 4.724 & $4.301 \mathrm{E}-07$ & 5.34E-07 & 0.81 \\
\hline 13 & 4.169 & $4.738 \mathrm{E}-07$ & 5.41E-07 & 0.88 \\
\hline 12 & 3.679 & $4.181 \mathrm{E}-07$ & $5.13 E-07$ & 0.82 \\
\hline 11 & 3.247 & $5.561 \mathrm{E}-07$ & $4.26 \mathrm{E}-07$ & 1.31 \\
\hline 10 & 2.865 & $4.864 \mathrm{E}-07$ & $6.08 \mathrm{E}-07$ & 0.80 \\
\hline 9 & 2.528 & $5.248 \mathrm{E}-07$ & $6.60 \mathrm{E}-07$ & 0.80 \\
\hline 8 & 2.231 & $1.159 \mathrm{E}-06$ & $7.58 \mathrm{E}-07$ & 1.53 \\
\hline 7 & 1.969 & $4.958 \mathrm{E}-07$ & $6.43 \mathrm{E}-07$ & 0.77 \\
\hline 6 & 1.738 & $6.181 \mathrm{E}-07$ & $5.96 \mathrm{E}-07$ & 1.04 \\
\hline 5 & 1.534 & $5.542 \mathrm{E}-07$ & $5.72 \mathrm{E}-07$ & 0.97 \\
\hline 4 & 1.353 & $4.681 \mathrm{E}-07$ & $5.71 \mathrm{E}-07$ & 0.82 \\
\hline 3 & 1.194 & $3.749 \mathrm{E}-07$ & $5.18 \mathrm{E}-07$ & 0.72 \\
\hline$\overline{2}$ & 1.054 & $3.309 \mathrm{E}-07$ & $6.19 \mathrm{E}-07$ & 0.53 \\
\hline 1 & 0.930 & $3.045 \mathrm{E}-07$ & $5.38 \mathrm{E}-07$ & 0.57 \\
\hline
\end{tabular}


BUGLE 96 VALIDATION WITH MORSE USING WATER

WSRC-TR-00349

AND IRON EXPERIMENTAL DATA FROM SINDBAD 97

Rev.0

Page 45 of 89

Table 5.1-18: BUGLE 80 and BUGLE 96 comparison. Detector at $35.56 \mathrm{~cm}$ from source.

\begin{tabular}{|c|c|c|c|}
\hline Group & Energy Boundary & \multicolumn{2}{|c|}{ Calculated/Experimental } \\
\hline & (MeV) & BUGLE 80 & BUGLE 96 \\
\hline 20 & 10 & 1.06 & 1.20 \\
19 & 8.825 & 1.09 & 1.12 \\
18 & 7.788 & 1.36 & 1.10 \\
17 & 6.873 & 1.14 & 0.87 \\
16 & 6.065 & 1.22 & 1.01 \\
15 & 5.353 & 1.06 & 0.81 \\
14 & 4.724 & 1.25 & 0.88 \\
13 & 4.169 & 1.17 & 0.82 \\
12 & 3.679 & 1.19 & 1.31 \\
11 & 3.247 & 0.82 & 0.80 \\
10 & 2.865 & 0.85 & 0.80 \\
9 & 2.528 & 0.87 & 1.53 \\
8 & 2.231 & 1.39 & 0.77 \\
7 & 1.969 & 0.97 & 1.04 \\
6 & 1.738 & 0.87 & 0.97 \\
5 & 1.534 & 0.81 & 0.82 \\
4 & 1.353 & 0.92 & 0.72 \\
3 & 1.194 & 0.68 & 0.53 \\
2 & 1.054 & 0.65 & 0.57 \\
1 & 0.930 & & \\
\hline
\end{tabular}

Figure 5.1-6: BUGLE 80 and BUGLE 96 data comparison. Detector at $35.56 \mathrm{~cm}$ from source.

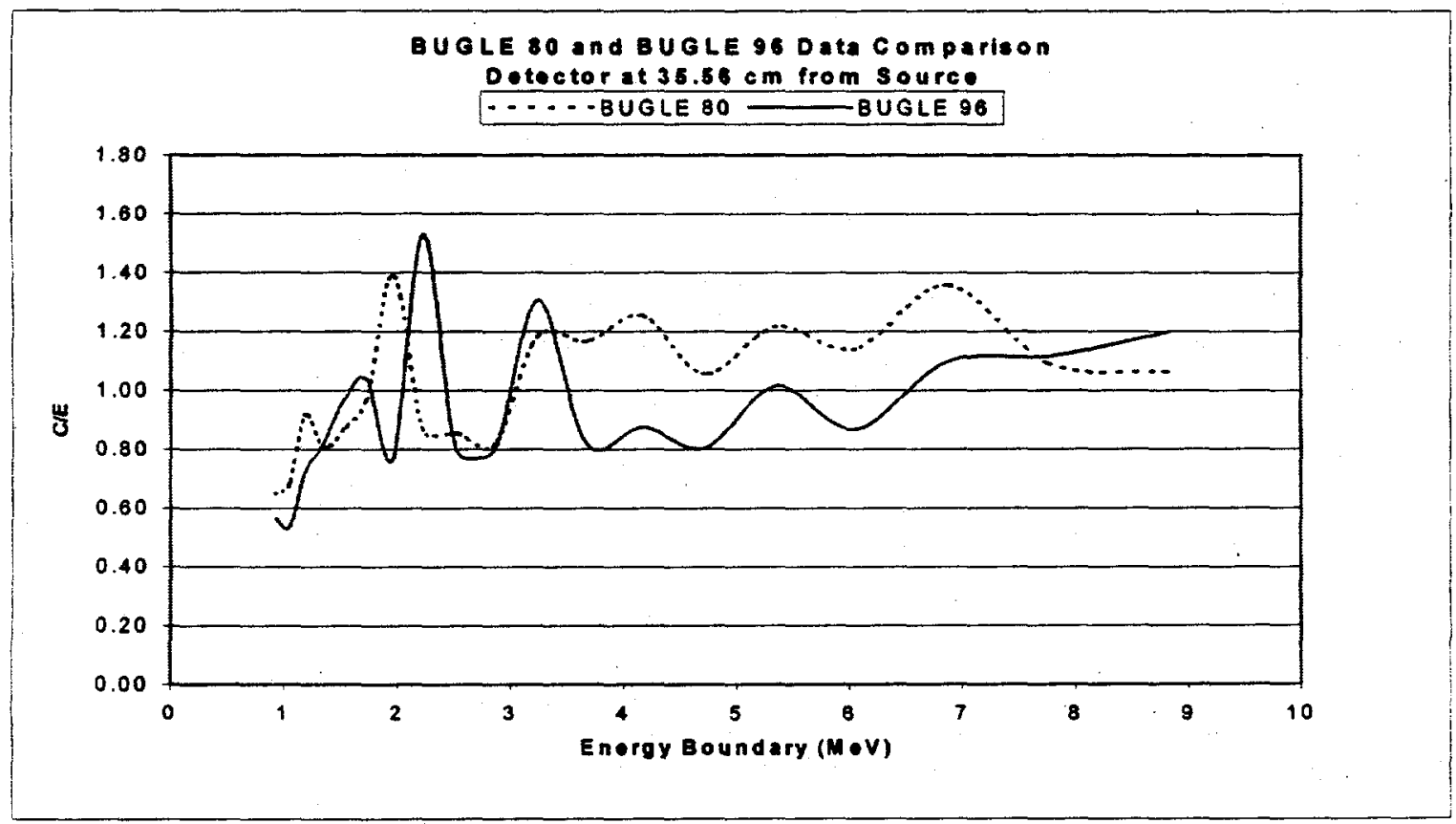


Table 5.1-19: BUGLE 80 results for a detector at a distance of $50.80 \mathrm{~cm}$ from source.

\begin{tabular}{|c|c|c|c|c|}
\hline \multirow[b]{2}{*}{ Group } & \multirow[b]{2}{*}{$\begin{array}{c}\text { Energy boundary } \\
(\mathrm{MeV})\end{array}$} & \multicolumn{2}{|c|}{ Flux/Lethargy per Source Neutron } & \multirow[b]{2}{*}{$\mathrm{C} / \mathrm{E}$} \\
\hline & & $\begin{array}{c}\text { Calculated (C) } \\
\left(\mathrm{cm}^{-2} / \mathrm{s}\right)\end{array}$ & $\begin{array}{c}\text { Experimental }(\mathrm{E}) \\
\left(\mathrm{cm}^{-2} / \mathrm{s}\right)\end{array}$ & \\
\hline 20 & 10 & & & \\
\hline 19 & 8.825 & $1.229 \mathrm{E}-08$ & $1.36 \mathrm{E}-08$ & 0.90 \\
\hline 18 & 7.788 & $2.186 \mathrm{E}-08$ & $1.77 \mathrm{E}-08$ & 1.24 \\
\hline 17 & 6.873 & $3.173 \mathrm{E}-08$ & $2.67 \mathrm{E}-08$ & 1.19 \\
\hline 16 & 6.065 & $3.418 \mathrm{E}-08$ & $3.90 \mathrm{E}-08$ & 0.88 \\
\hline 15 & 5.353 & $4.307 \mathrm{E}-08$ & $4.61 \mathrm{E}-08$ & 0.93 \\
\hline 14 & 4.724 & $4.323 \mathrm{E}-08$ & $4.51 \mathrm{E}-08$ & 0.96 \\
\hline 13 & 4.169 & $4.552 \mathrm{E}-08$ & $5.26 \mathrm{E}-08$ & 0.87 \\
\hline 12 & 3.679 & $4.017 \mathrm{E}-08$ & $3.67 \mathrm{E}-08$ & 1.09 \\
\hline 11 & 3.247 & $4.022 \mathrm{E}-08$ & $4.03 \mathrm{E}-08$ & 1.00 \\
\hline 10 & 2.865 & $3.615 \mathrm{E}-08$ & $4.39 \mathrm{E}-08$ & 0.82 \\
\hline 9 & 2.528 & $3.632 \mathrm{E}-08$ & 4.87E-08 & 0.75 \\
\hline 8 & 2.231 & $8.064 \mathrm{E}-08$ & $5.60 \mathrm{E}-08$ & 1.44 \\
\hline 7 & 1.969 & $2.791 \mathrm{E}-08$ & $4.39 \mathrm{E}-08$ & 0.64 \\
\hline 6 & 1.738 & $2.951 \mathrm{E}-08$ & $4.15 \mathrm{E}-08$ & 0.71 \\
\hline 5 & 1.534 & $4.540 \mathrm{E}-08$ & $4.14 \mathrm{E}-08$ & 1.10 \\
\hline 4 & 1.353 & $5.155 \mathrm{E}-08$ & $4.00 \mathrm{E}-08$ & 1.29 \\
\hline 3 & 1.194 & $1.959 \mathrm{E}-08$ & $3.77 \mathrm{E}-08$ & 0.52 \\
\hline 2 & 1.054 & $1.729 \mathrm{E}-08$ & $4.23 \mathrm{E}-08$ & 0.41 \\
\hline 1 & 0.930 & $3.317 \mathrm{E}-08$ & $4.41 \mathrm{E}-08$ & 0.75 \\
\hline
\end{tabular}


BUGLE 96 VALIDATION WITH MORSE USNNG WATER

WSRC-TR-00349 AND IRON EXPERMMENTAL DATA FROM SINDBAD 97

Rev.0

Page 47 of 89

Table 5.1-20: BUGLE 96 results for a detector at a distance of $50.80 \mathrm{~cm}$ from source

\begin{tabular}{|c|c|c|c|c|}
\hline \multirow[b]{2}{*}{ Group } & \multirow[b]{2}{*}{$\begin{array}{l}\text { Energy boundary } \\
\text { (MeV) }\end{array}$} & \multicolumn{2}{|c|}{ Flux/Lethargy per Source Neutron } & \multirow[b]{2}{*}{$\mathrm{C} / \mathrm{E}$} \\
\hline & & $\begin{array}{c}\text { Calculated (C) } \\
\left(\mathrm{cm}^{-2} / \mathrm{s}\right)\end{array}$ & $\begin{array}{c}\text { Experimental (E) } \\
\left(\mathrm{cm}^{-2} / \mathrm{s}\right)\end{array}$ & \\
\hline 20 & 10 & & & \\
\hline 19 & 8.825 & $1.562 \mathrm{E}-08$ & $1.36 \mathrm{E}-08$ & 1.15 \\
\hline 18 & 7.788 & $2.037 \mathrm{E}-08$ & $1.77 \mathrm{E}-08$ & 1.15 \\
\hline 17 & 6.873 & $2.791 \mathrm{E}-08$ & $2.67 \mathrm{E}-08$ & 1.05 \\
\hline 16 & 6.065 & $2.989 \mathrm{E}-08$ & $3.90 \mathrm{E}-08$ & 0.77 \\
\hline 15 & 5.353 & $4.418 \mathrm{E}-08$ & $4.61 \mathrm{E}-08$ & 0.96 \\
\hline 14 & 4.724 & $4.548 \mathrm{E}-08$ & $4.51 \mathrm{E}-08$ & 1.01 \\
\hline 13 & 4.169 & $4.929 \mathrm{E}-08$ & $5.26 \mathrm{E}-08$ & 0.94 \\
\hline 12 & 3.679 & $4.350 \mathrm{E}-08$ & $3.67 \mathrm{E}-08$ & 1.19 \\
\hline 11 & 3.247 & $3.443 \mathrm{E}-08$ & $4.03 \mathrm{E}-08$ & 0.85 \\
\hline 10 & 2.865 & $2.723 \mathrm{E}-08$ & $4.39 \mathrm{E}-08$ & 0.62 \\
\hline 9 & 2.528 & $2.727 \mathrm{E}-08$ & $4.87 \mathrm{E}-08$ & 0.56 \\
\hline 8 & 2.231 & $3.271 \mathrm{E}-08$ & $5.60 \mathrm{E}-08$ & 0.58 \\
\hline$\overline{7}$ & 1.969 & $3.377 \mathrm{E}-08$ & $4.39 \mathrm{E}-08$ & 0.77 \\
\hline 6 & 1.738 & $2.099 \mathrm{E}-08$ & $4.15 \mathrm{E}-08$ & 0.51 \\
\hline 5 & 1.534 & $1.496 \mathrm{E}-08$ & $4.14 \mathrm{E}-08$ & 0.36 \\
\hline 4 & 1.353 & $1.225 \mathrm{E}-08$ & $4.00 \mathrm{E}-08$ & 0.31 \\
\hline 3 & 1.194 & $3.467 \mathrm{E}-08$ & $3.77 \mathrm{E}-08$ & 0.92 \\
\hline 2 & 1.054 & $3.060 \mathrm{E}-08$ & $4.23 \mathrm{E}-08$ & 0.72 \\
\hline 1 & 0.930 & $2.937 \mathrm{E}-08$ & $4.41 \mathrm{E}-08$ & 0.67 \\
\hline
\end{tabular}


BUGLE 96 VALIDATION WITH MORSE USING WATER AND IRON EXPERIMENTAL DATA FROM SINDBAD 97

WSRC-TR-00349

Rev.0

Page 48 of 89

Table 5.1-21: BUGLE 80 and BUGLE 96 comparison. Detector at $50.86 \mathrm{~cm}$ from source.

\begin{tabular}{|c|c|c|c|}
\hline Group & Energy Boundary & \multicolumn{2}{|c|}{ Experimental/Calculated } \\
\hline & $(\mathrm{MeV})$ & BUGLE 80 & BUGLE 96 \\
\hline 20 & 10 & & \\
19 & 8.825 & 0.90 & 1.15 \\
18 & 7.788 & 1.24 & 1.15 \\
17 & 6.873 & 1.19 & 1.05 \\
16 & 6.065 & 0.88 & 0.77 \\
15 & 5.353 & 0.93 & 0.96 \\
14 & 4.724 & 0.96 & 1.01 \\
13 & 4.169 & 0.87 & 0.94 \\
12 & 3.679 & 1.09 & 1.19 \\
11 & 3.247 & 1.00 & 0.85 \\
10 & 2.865 & 0.82 & 0.62 \\
9 & 2.528 & 0.75 & 0.56 \\
8 & 2.231 & 1.44 & 0.58 \\
7 & 1.969 & 0.64 & 0.77 \\
6 & 1.738 & 0.71 & 0.51 \\
5 & 1.534 & 1.10 & 0.36 \\
4 & 1.353 & 1.29 & 0.31 \\
3 & 1.194 & 0.52 & 0.92 \\
2 & 1.054 & 0.41 & 0.72 \\
1 & 0.930 & 0.75 & 0.67 \\
\hline
\end{tabular}

Figure 5.1-7: BUGLE 80 and BUGLE 96 data comparison. Detector at $50.80 \mathrm{~cm}$ from source.

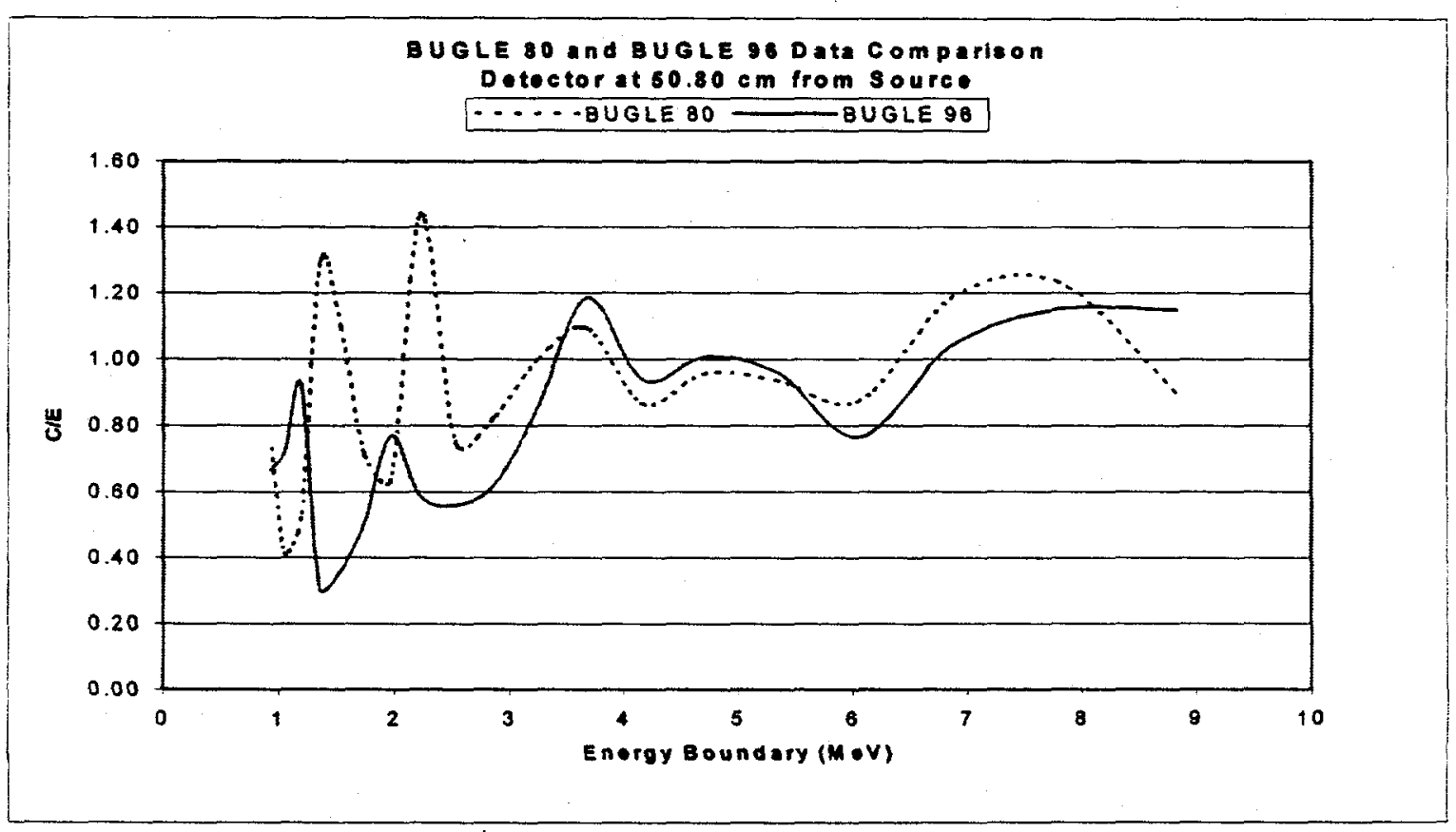


BUGLE 96 VALIDATION WITH MORSE USING WATER

AND IRON EXPERIMENTAL DATA FROM SINDBAD 97

WSRC-TR-00349

Rev.0

Page 49 of 89

\subsection{Karlsruhe Iron Sphere Benchmark Experiment}

Three types of proton recoil detectors were used to measure neutron spectra at different overlapping energy ranges for each of the six iron spheres. Detector 1 measured neutron spectrum from approximately $60 \mathrm{KeV}$ to $850 \mathrm{KeV}$, the second detector measured spectrum from $300 \mathrm{KeV}$ to $2300 \mathrm{KeV}$ and the third measured spectrum from 900 to $5000 \mathrm{KeV}$. Results are tabulated for each of the six iron spheres. Tables 5.2-1 to 5.2-12 give the calculated and experimental values of neutron per source neutron per lethargy unit as well as the corresponding $\mathrm{C} / \mathrm{E}$ ratios in each energy interval. For each sphere size, a graphical representations of the $\mathrm{C} / \mathrm{E}$ ratios for the three detectors can be seen in Figures 5.2-1 to 5.2-18.

For detector 1, the calculated values lie within $20 \%$ of the experimental values from about 60 $\mathrm{KeV}$ to $300 \mathrm{KeV}$ range. The calculated values were off by more than $20 \%$ in the $300 \mathrm{KeV}$ to $500 \mathrm{KeV}$ range, where a pronounced type of structure was observed. The calculated results were again in the $20 \%$ range with the measured value from $500 \mathrm{KeV}$ to $700 \mathrm{KeV}$ range.

For detector 2 , the calculated values were within $20 \%$ of the measured value throughout most of the energy range of detector 2 except near the upper energy band of $2000 \mathrm{KeV}$. For detector 3 , the calculated values were mostly higher than the measured values in the $900 \mathrm{KeV}$ to $4000 \mathrm{KeV}$ range and the $\mathrm{C} / \mathrm{E}$ ratios lie between 1.0 to 1.1 within this range. Beyond the range of 4000 $\mathrm{KeV}$, the calculated values were increasingly higher than the experimental results.

The calculated results with BUGLE-96 and BUGLE-80 were very close and differ by no more than $10 \%$. The trend for each detector remains the same for different iron spheres (diameters vary from $15 \mathrm{~cm}$ to $40 \mathrm{~cm}$ ) 
Table 5.2-1: Detector Measurements for $15 \mathrm{~cm}$ diameter Iron Sphere using BUGLE 80 cross section library.

\begin{tabular}{|c|c|c|c|c|c|c|c|c|c|c|c|}
\hline \multicolumn{4}{|c|}{ Detector 1} & \multicolumn{4}{|c|}{ Detector 2} & \multicolumn{4}{|c|}{ Detector 3} \\
\hline $\begin{array}{l}\text { Mid Energy } \\
\text { (keV) }\end{array}$ & $\begin{array}{l}\text { Calc. } \\
\text { (C) }\end{array}$ & $\begin{array}{c}\text { Exp. } \\
\text { (E) }\end{array}$ & $\mathrm{C} / \mathrm{E}$ & $\begin{array}{c}\text { Mid Energy } \\
(\mathrm{keV})\end{array}$ & $\begin{array}{l}\text { Calc. } \\
\text { (C) }\end{array}$ & $\begin{array}{l}\text { Exp. } \\
\text { (E) }\end{array}$ & $\mathrm{C} / \mathrm{E}$ & $\begin{array}{l}\text { Mid Energy } \\
(\mathrm{keV})\end{array}$ & $\begin{array}{l}\text { Calc. } \\
\text { (C) }\end{array}$ & $\begin{array}{l}\text { Exp. } \\
\text { (E) }\end{array}$ & $\mathrm{C} / \mathrm{E}$ \\
\hline $6.775 \mathrm{E}+01$ & 0.0219 & 0.0348 & 0.63 & $3.081 \mathrm{E}+02$ & 0.2714 & 0.2275 & 1.19 & $9.165 \mathrm{E}+02$ & 0.3932 & 0.3811 & 1.03 \\
\hline $7.285 \mathrm{E}+01$ & 0.0252 & 0.0499 & 0.51 & $3.317 \mathrm{E}+02$ & 0.2633 & 0.2448 & 1.08 & $9.870 \mathrm{E}+02$ & 0.3966 & 0.3853 & 1.03 \\
\hline $7.850 \mathrm{E}+01$ & 0.0290 & 0.0558 & 0.52 & $3.568 \mathrm{E}+02$ & 0.2622 & 0.2592 & 1.01 & $1.063 \mathrm{E}+03$ & 0.4129 & 0.3769 & 1.10 \\
\hline $8.415 \mathrm{E}+01$ & 0.0333 & 0.0498 & 0.67 & $3.833 \mathrm{E}+02$ & 0.2579 & 0.2117 & 1.22 & $1.142 \mathrm{E}+03$ & 0.4287 & 0.4001 & 1.07 \\
\hline $9.035 \mathrm{E}+01$ & 0.0381 & 0.0521 & 0.73 & $4.110 \mathrm{E}+02$ & 0.2506 & 0.1786 & 1.40 & $1.226 \mathrm{E}+03$ & $\overline{0.4307}$ & 0.4169 & 1.03 \\
\hline $9.710 \mathrm{E}+01$ & 0.0424 & 0.0367 & 1.15 & $4.423 \mathrm{E}+02$ & 0.2469 & 0.1858 & 1.33 & $1.320 \mathrm{E}+03$ & 0.4133 & 0.3769 & 1.10 \\
\hline $1.044 \mathrm{E}+02$ & 0.0474 & 0.0461 & 1.03 & $4.762 \mathrm{E}+02$ & 0.2692 & 0.2751 & 0.98 & $1.419 \mathrm{E}+03$ & 0.3884 & 0.3706 & 1.05 \\
\hline $1.123 \mathrm{E}+02$ & 0.0531 & 0.0680 & 0.78 & $5.130 \mathrm{E}+02$ & 0.2934 & 0.2837 & 1.03 & $1.524 \mathrm{E}+03$ & 0.3616 & 0.3453 & 1.05 \\
\hline $1.208 E+02$ & 0.0596 & 0.0646 & 0.92 & $5.514 \mathrm{E}+02$ & 0.3201 & 0.2895 & 1.11 & $1.638 \mathrm{E}+03$ & 0.3626 & 0.3537 & 1.03 \\
\hline $1.299 \mathrm{E}+02$ & 0.0670 & 0.0800 & 0.84 & $5.927 \mathrm{E}+02$ & 0.3711 & 0.3586 & 1.03 & $1.761 \mathrm{E}+03$ & 0.3600 & 0.3601 & 1.00 \\
\hline $1.395 \mathrm{E}+02$ & 0.0752 & 0.0903 & 0.83 & $6.369 \mathrm{E}+02$ & 0.4309 & 0.4709 & 0.92 & $1.896 \mathrm{E}+03$ & 0.3411 & 0.3348 & 1.02 \\
\hline $1.496 \mathrm{E}+02$ & 0.0845 & 0.0750 & 1.13 & $6.840 \mathrm{E}+02$ & 0.4759 & 0.5905 & 0.81 & $2.039 \mathrm{E}+03$ & 0.3117 & 0.2906 & 1.07 \\
\hline $1.609 \mathrm{E}+02$ & 0.0706 & 0.0624 & 1.13 & $7.356 \mathrm{E}+02$ & 0.3947 & $\overline{0.3082}$ & 1.28 & $2.191 \mathrm{E}+03$ & 0.3090 & 0.2842 & 1.09 \\
\hline $1.734 \mathrm{E}+02$ & 0.0734 & 0.1140 & 0.64 & $7.917 E+02$ & 0.3123 & 0.3456 & 0.90 & $2.355 \mathrm{E}+03$ & 0.3157 & 0.2779 & 1.14 \\
\hline $1.863 \mathrm{E}+02$ & 0.1086 & 0.1368 & 0.79 & $8.521 \mathrm{E}+02$ & 0.3445 & 0.4004 & 0.86 & $2.533 \mathrm{E}+03$ & 0.2572 & 0.2484 & 1.04 \\
\hline $2.004 \mathrm{E}+02$ & 0.1164 & 0.1072 & 1.09 & $9.155 E+02$ & 0.3793 & 0.3672 & 1.03 & $2.726 \mathrm{E}+03$ & 0.2358 & 0.2337 & 1.01 \\
\hline $2.157 \mathrm{E}+02$ & 0.1221 & 0.1268 & 0.96 & $9.850 \mathrm{E}+02$ & 0.3964 & 0.3629 & 1.09 & $2.931 \mathrm{E}+03$ & 0.2161 & 0.2076 & 1.04 \\
\hline $2.315 \mathrm{E}+02$ & 0.1334 & 0.1354 & 0.99 & $1.060 \mathrm{E}+03$ & 0.4128 & 0.3860 & 1.07 & $3.150 \mathrm{E}+03$ & 0.1909 & 0.1916 & 1.00 \\
\hline $2.484 \mathrm{E}+02$ & 0.1481 & 0.1226 & 1.21 & $1.139 \mathrm{E}+03$ & 0.4289 & 0.4033 & 1.06 & $3.387 \mathrm{E}+03$ & 0.1623 & 0.1619 & 1.00 \\
\hline $2.677 \mathrm{E}+02$ & 0.1530 & 0.1677 & 0.91 & $1.225 \mathrm{E}+03$ & 0.4298 & 0.3384 & 1.27 & $3.641 \mathrm{E}+03$ & 0.1523 & 0.1453 & 1.05 \\
\hline $2.880 \mathrm{E}+02$ & 0.1652 & 0.1876 & 0.88 & $1.316 \mathrm{E}+03$ & 0.4145 & 0.3730 & 1.11 & $3.916 \mathrm{E}+03$ & 0.1380 & 0.1303 & 1.06 \\
\hline $3.095 \mathrm{E}+02$ & 0.1436 & 0.2436 & 0.59 & $1.415 E+03$ & 0.3899 & 0.3888 & 1.00 & $4.212 \mathrm{E}+03$ & 0.1185 & 0.1128 & 1.05 \\
\hline $3.326 \mathrm{E}+02$ & 0.1546 & 0.2358 & 0.66 & $1.523 \mathrm{E}+03$ & 0.3617 & 0.3485 & 1.04 & $4.527 \mathrm{E}+03$ & 0.1048 & 0.0874 & 1.20 \\
\hline $3.575 \mathrm{E}+02$ & 0.1670 & 0.2788 & 0.60 & $1.638 \mathrm{E}+03$ & 0.3629 & 0.4076 & 0.89 & $4.869 \mathrm{E}+03$ & 0.0935 & 0.0697 & 1.34 \\
\hline $3.846 \mathrm{E}+02$ & 0.3162 & 0.2462 & 1.28 & $1.760 \mathrm{E}+03$ & 0.3606 & 0.3269 & 1.10 & $5.238 \mathrm{E}+03$ & 0.0754 & 0.0484 & 1.56 \\
\hline $4.139 \mathrm{E}+02$ & 0.3371 & 0.1745 & 1.93 & $1.893 \mathrm{E}+03$ & 0.3425 & 0.3269 & 1.05 & & & & \\
\hline $4.450 \mathrm{E}+02$ & 0.3641 & 0.1980 & 1.84 & $2.036 \mathrm{E}+03$ & 0.3129 & 0.2578 & 1.21 & & & & \\
\hline $4.783 E+02$ & 0.3938 & 0.2762 & 1.43 & $2.189 \mathrm{E}+03$ & 0.3093 & 0.2621 & 1.18 & & & & \\
\hline $5.144 E+02$ & 0.2973 & 0.2462 & 1.21 & $2.354 \mathrm{E}+03$ & 0.3499 & 0.2318 & 1.51 & & & & \\
\hline $5.534 \mathrm{E}+02$ & 0.3185 & 0.3087 & 1.03 & & & & & & & & \\
\hline $5.952 \mathrm{E}+02$ & 0.3568 & 0.4012 & 0.89 & & & & & & & & \\
\hline $6.398 \mathrm{E}+02$ & 0.3853 & 0.4273 & 0.90 & & & & & & & & \\
\hline $6.878 \mathrm{E}+02$ & 0.4131 & 0.3817 & 1.08 & & & & & & & & \\
\hline $7.392 \mathrm{E}+02$ & 0.5554 & 0.4038 & 1.38 & & & & & & & & \\
\hline $7.951 \mathrm{E}+02$ & 0.5928 & 0.3960 & 1.50 & & & & & & & BUG80/15 & \\
\hline
\end{tabular}




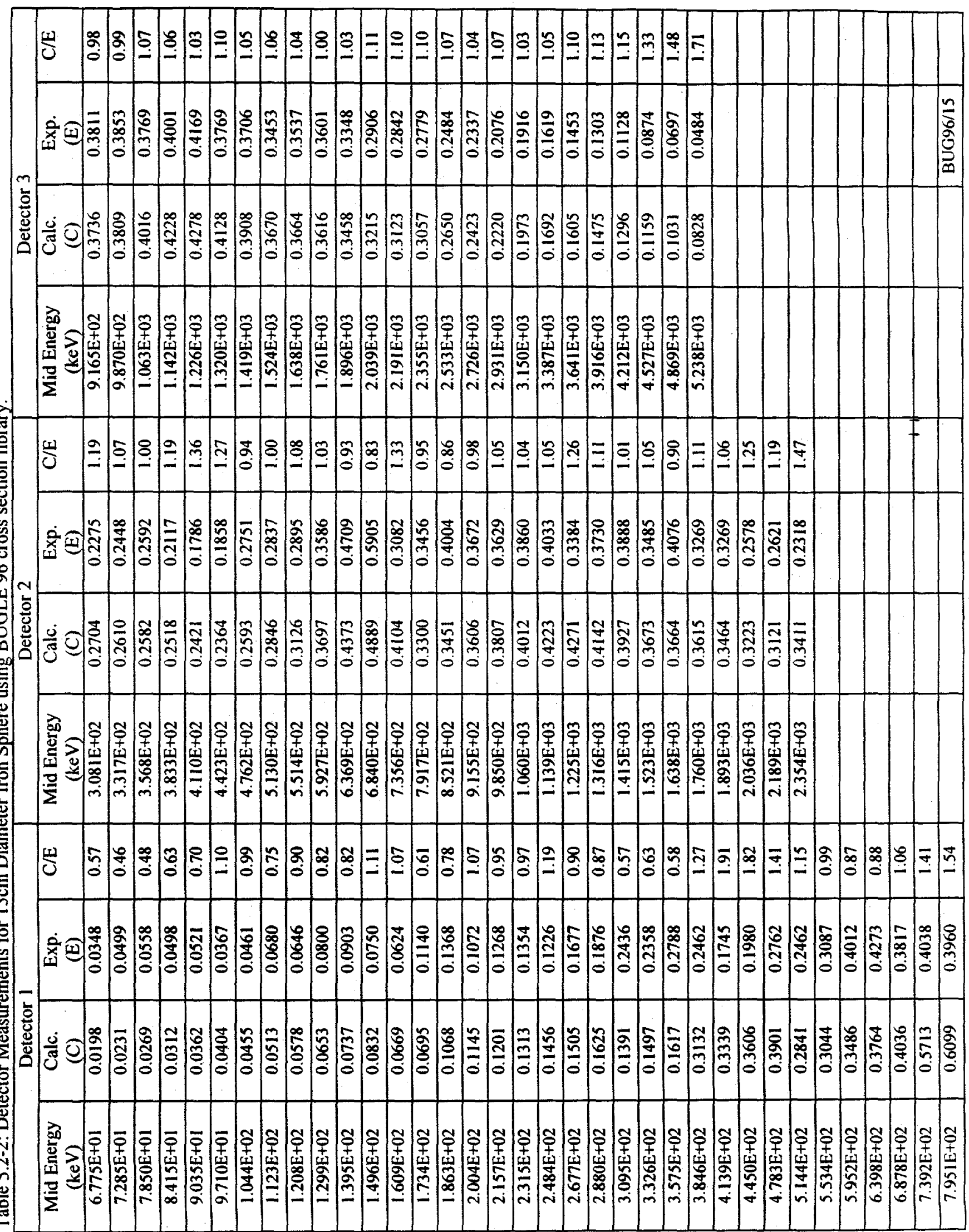


Figure 5.2-1: Detector 1 Data Comparison for $15 \mathrm{~cm}$ Sphere

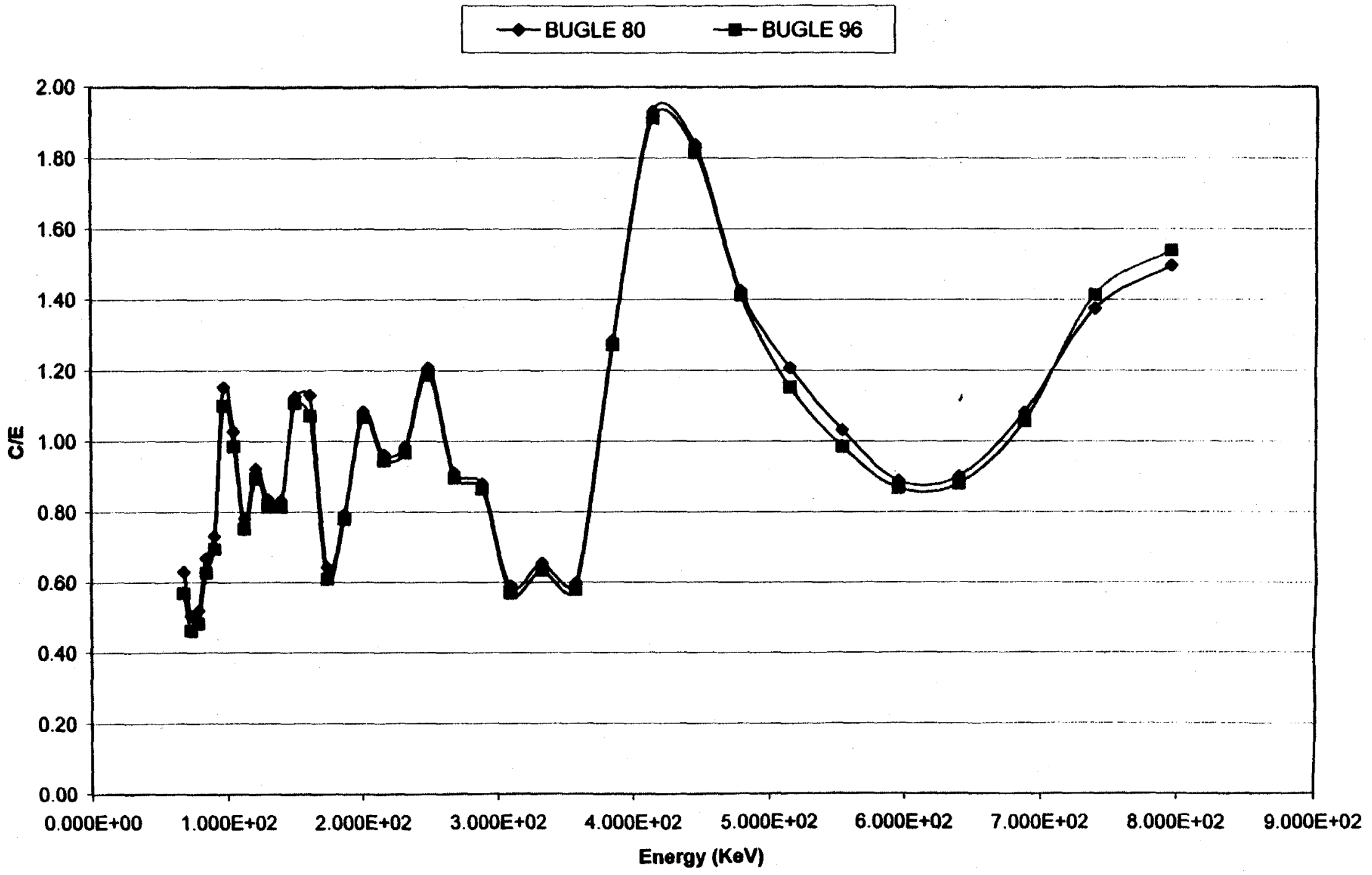


Figure 5.2-2: Detector 2 data comparison for $15 \mathrm{~cm}$ sphere.

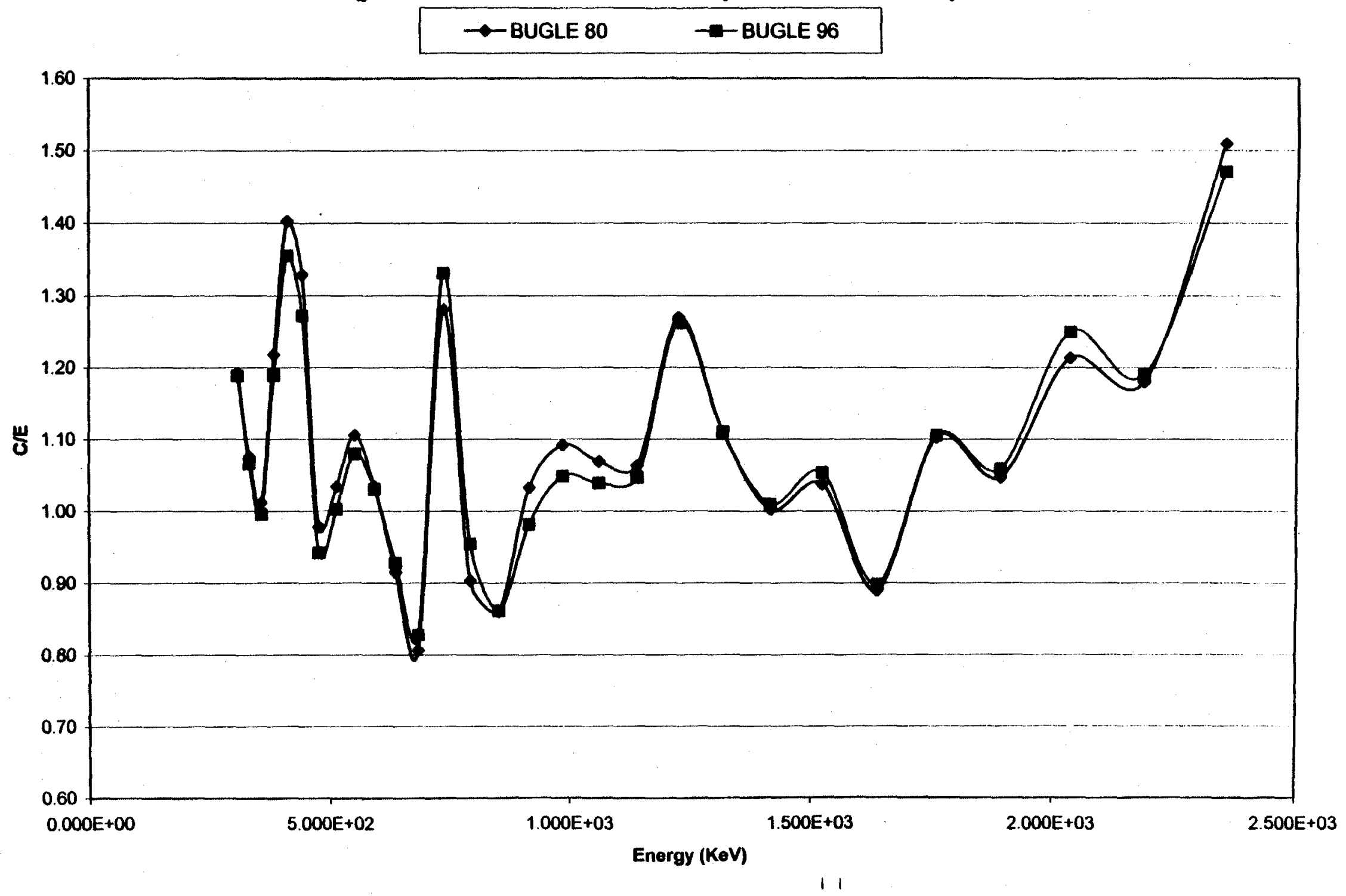


Figure 5.2-3: Detector 3 Data Comparison for $15 \mathrm{~cm}$ Sphere

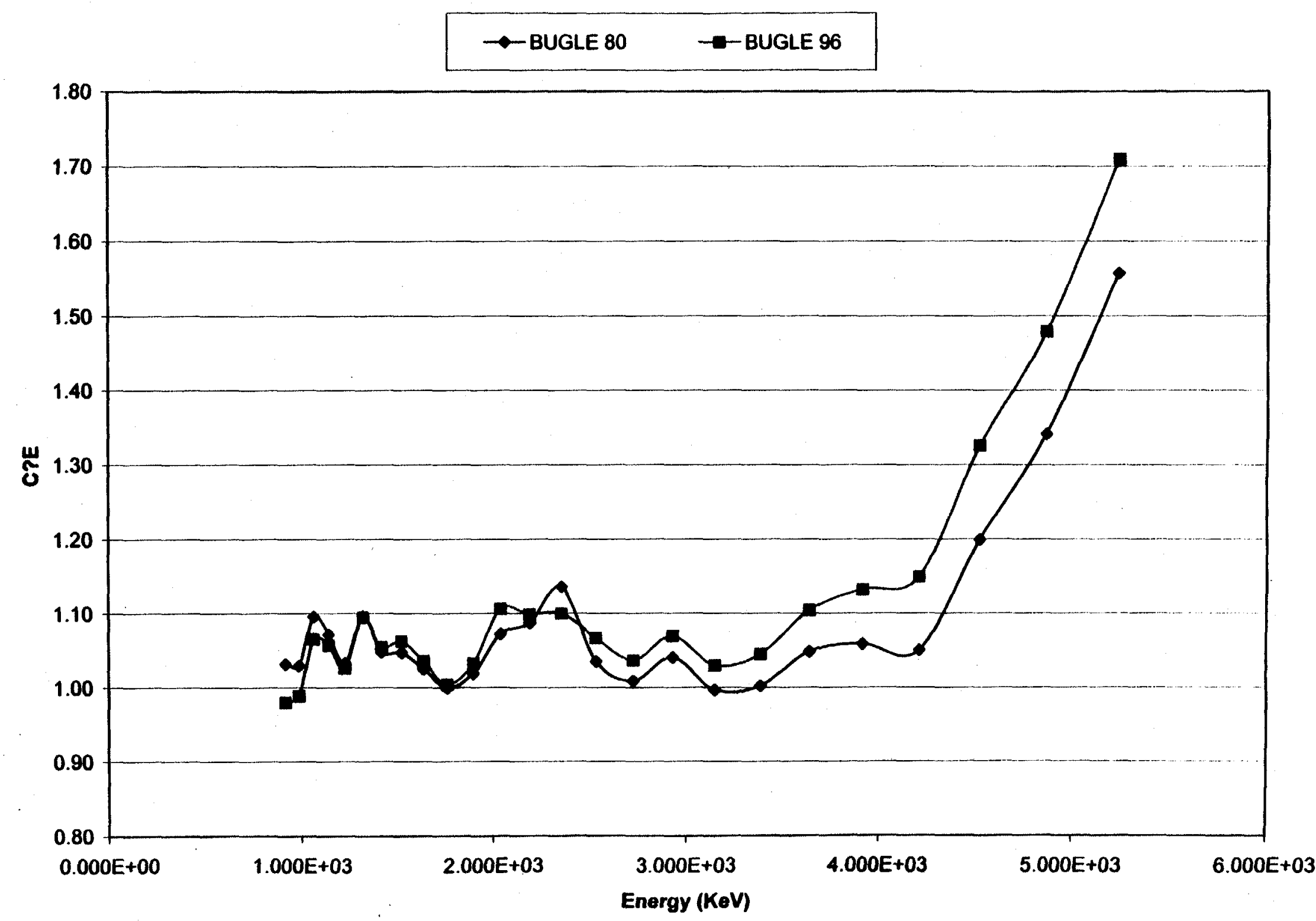


(1)

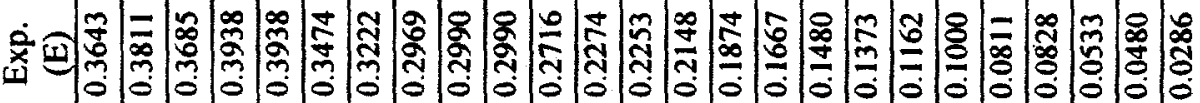
m

豙

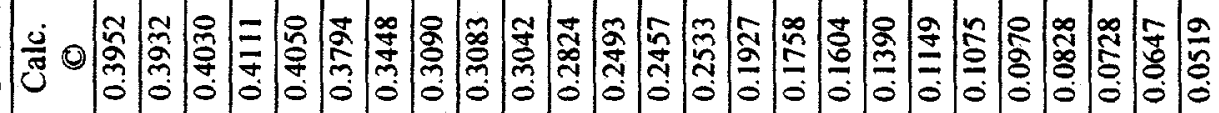

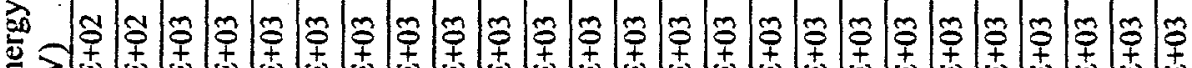

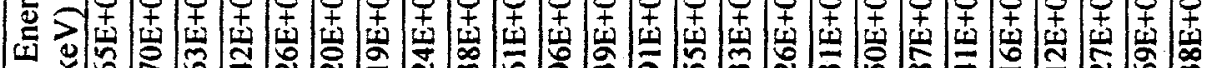

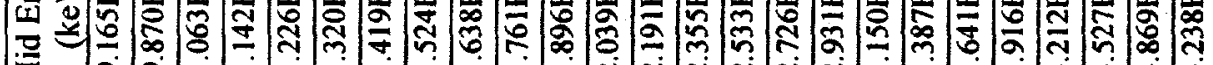



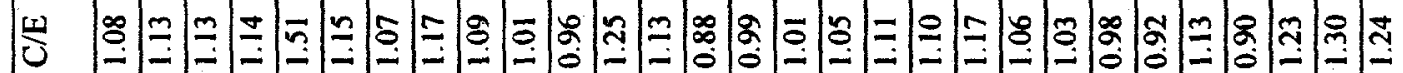

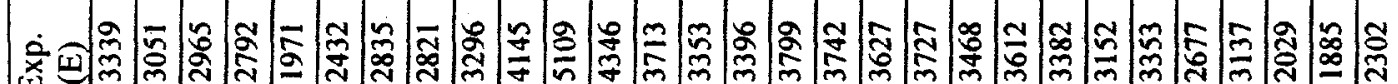
4.

(2)

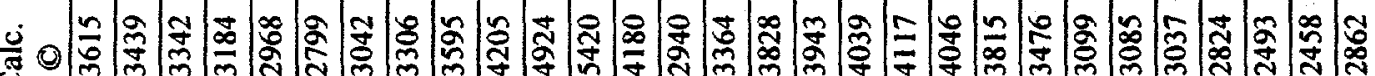

$\frac{5}{2}$

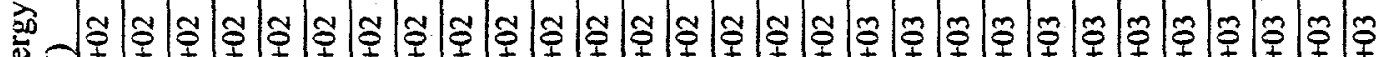

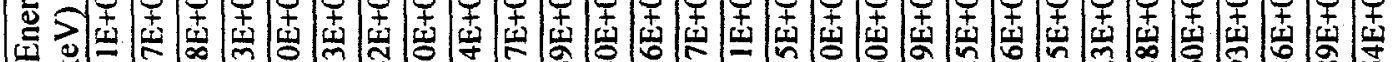

氙

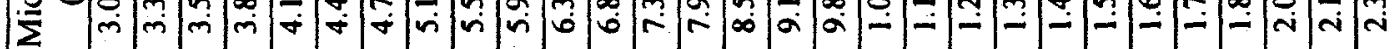

过

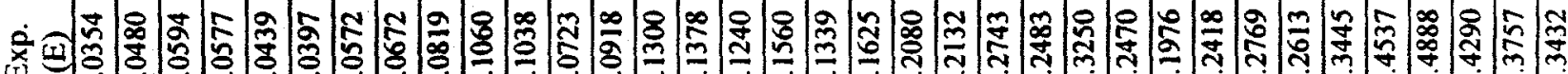
$-$

(2)

ن

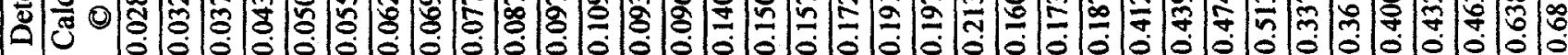

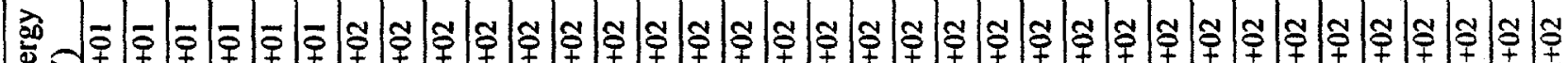

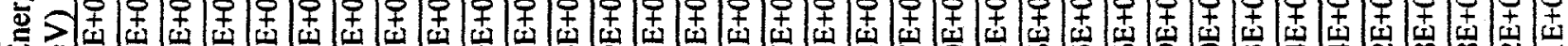

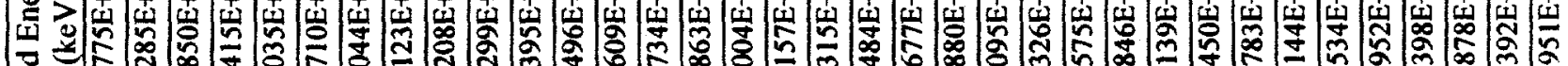

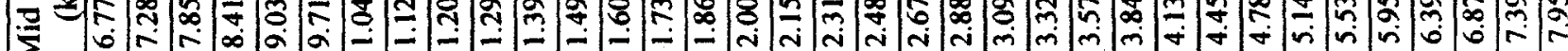


Table 5.2-4: Detector Measurements for $20 \mathrm{~cm}$ Diameter Iron Sphere using BUGLE 96 cross section library.

\begin{tabular}{|c|c|c|c|c|c|c|c|c|c|c|c|}
\hline \multicolumn{4}{|c|}{ Detector 1} & \multicolumn{4}{|c|}{ Detector 2} & \multicolumn{4}{|c|}{ Detector 3} \\
\hline $\begin{array}{c}\text { Mid Energy } \\
\text { (keV) }\end{array}$ & $\begin{array}{c}\text { Calc. } \\
\text { (0) }\end{array}$ & $\begin{array}{l}\text { Exp. } \\
\text { (E) }\end{array}$ & $\mathrm{C} / \mathrm{E}$ & $\begin{array}{l}\text { Mid Energy } \\
\text { (keV) }\end{array}$ & $\begin{array}{c}\text { Calc. } \\
\text { (C) }\end{array}$ & $\begin{array}{l}\text { Exp. } \\
\text { (E) }\end{array}$ & $\mathrm{C} / \mathrm{E}$ & $\begin{array}{l}\text { Mid Energy } \\
\text { (keV) }\end{array}$ & $\begin{array}{l}\text { Calc. } \\
\text { (C) }\end{array}$ & $\begin{array}{l}\text { Exp. } \\
\text { (E) }\end{array}$ & $\mathrm{C} / \mathrm{E}$ \\
\hline $6.775 \mathrm{E}+01$ & 0.0250 & 0.0354 & 0.71 & $3.081 \mathrm{E}+02$ & 0.3649 & 0.3339 & 1.09 & $9.165 \mathrm{E}+02$ & 0.3696 & 0.3643 & 1.01 \\
\hline $7.285 \mathrm{E}+0.1$ & 0.0294 & 0.0480 & 0.61 & $3.317 \mathrm{E}+02$ & 0.3448 & 0.3051 & 1.13 & $9.870 \mathrm{E}+02$ & 0.3729 & 0.3811 & 0.98 \\
\hline $7.850 \mathrm{E}+01$ & 0.0346 & 0.0594 & 0.58 & $3.568 \mathrm{E}+02$ & 0.3320 & 0.2965 & 1.12 & $1.063 \mathrm{E}+03$ & 0.3885 & 0.3685 & 1.05 \\
\hline $8.415 \mathrm{E}+01$ & 0.0404 & 0.0577 & 0.70 & $3.833 \mathrm{E}+02$ & 0.3126 & 0.2792 & 1.12 & $1.142 E+03$ & 0.4037 & 0.3938 & 1.03 \\
\hline $9.035 \mathrm{E}+01$ & 0.0472 & 0.0439 & 1.07 & $4.110 \mathrm{E}+02$ & 0.2867 & 0.1971 & 1.45 & $1.226 \mathrm{E}+03$ & 0.4028 & 0.3938 & 1.02 \\
\hline $9.710 \mathrm{E}+01$ & 0.0204 & 0.0397 & 0.52 & $4.423 \mathrm{E}+02$ & 0.2667 & 0.2432 & 1.10 & $1.320 \mathrm{E}+03$ & 0.3806 & 0.3474 & 1.10 \\
\hline $1.044 \mathrm{E}+02$ & 0.0591 & 0.0572 & 1.03 & $4.762 \mathrm{E}+02$ & 0.2926 & 0.2835 & 1.03 & $1.419 \mathrm{E}+03$ & 0.3504 & 0.3222 & 1.09 \\
\hline $1.123 \mathrm{E}+02$ & 0.0666 & 0.0672 & 0.99 & $5.130 \mathrm{E}+02$ & 0.3210 & 0.2821 & 1.14 & $1.524 \mathrm{E}+03$ & 0.3182 & 0.2969 & 1.07 \\
\hline $1.208 \mathrm{E}+02$ & 0.0751 & 0.0819 & 0.92 & $5.514 \mathrm{E}+02$ & 0.3526 & 0.3296 & 1.07 & $1.638 \mathrm{E}+03$ & 0.3149 & 0.2990 & 1.05 \\
\hline $1.299 \mathrm{E}+02$ & 0.0847 & 0.1060 & 0.80 & $5.927 \mathrm{E}+02$ & 0.4234 & 0.4145 & 1.02 & $1.761 \mathrm{E}+03$ & 0.3074 & 0.2990 & 1.03 \\
\hline $1.395 \mathrm{E}+02$ & 0.0955 & 0.1038 & 0.92 & $6.369 \mathrm{E}+02$ & 0.5075 & 0.5109 & 0.99 & $1.896 \mathrm{E}+03$ & 0.2888 & 0.2716 & 1.06 \\
\hline $1.496 \mathrm{E}+02$ & 0.1078 & 0.0723 & 1.49 & $6.840 \mathrm{E}+02$ & 0.5690 & 0.4346 & 1.31 & $2.039 \mathrm{E}+03$ & 0.2616 & 0.2274 & 1.15 \\
\hline $1.609 \mathrm{E}+02$ & 0.0870 & 0.0918 & 0.95 & $7.356 \mathrm{E}+02$ & 0.4458 & 0.3713 & 1.20 & $2.191 \mathrm{E}+03$ & 0.2513 & 0.2253 & 1.12 \\
\hline $1.734 \mathrm{E}+02$ & 0.0904 & 0.1300 & 0.70 & $7.917 \mathrm{E}+02$ & 0.3186 & 0.3353 & 0.95 & $2.355 \mathrm{E}+03$ & 0.2456 & 0.2148 & 1.14 \\
\hline $1.863 \mathrm{E}+02$ & 0.1385 & 0.1378 & 1.01 & $8.521 E+02$ & 0.3369 & 0.3396 & 0.99 & $2.533 \mathrm{E}+03$ & 0.2020 & 0.1874 & 1.08 \\
\hline $2.004 \mathrm{E}+02$ & 0.1484 & 0.1240 & 1.20 & $9.155 \mathrm{E}+02$ & 0.3560 & 0.3799 & 0.94 & $2.726 \mathrm{E}+03$ & 0.1824 & 0.1667 & $1: 09$ \\
\hline $2.157 \mathrm{E}+02$ & 0.1557 & 0.1560 & 1.00 & $9.850 \mathrm{E}+02$ & 0.3725 & 0.3742 & 1.00 & $2.931 E+03$ & 0.1658 & 0.1480 & 1.12 \\
\hline $2.315 \mathrm{E}+02$ & 0.1702 & 0.1339 & 1.27 & $1.060 \mathrm{E}+03$ & 0.3884 & 0.3627 & 1.07 & $3.150 \mathrm{E}+03$ & 0.1454 & 0.1373 & 1.06 \\
\hline $2.484 E+02$ & 0.1889 & 0.1625 & 1.16 & $1.139 \mathrm{E}+03$ & 0.4042 & 0.3727 & 1.08 & $3.387 \mathrm{E}+03$ & 0.1221 & 0.1162 & 1.05 \\
\hline $2.677 E+02$ & 0.1951 & 0.2080 & 0.94 & $1.225 \mathrm{E}+03$ & 0.4028 & 0.3468 & 1.16 & $3.641 \mathrm{E}+03$ & 0.1156 & 0.1000 & 1.16 \\
\hline $2.880 \mathrm{E}+02$ & 0.2107 & 0.2132 & 0.99 & $1.316 \mathrm{E}+03$ & 0.3825 & 0.3612 & 1.06 & $3.916 \mathrm{E}+03$ & 0.1060 & 0.0811 & 1.31 \\
\hline $3.095 \mathrm{E}+02$ & 0.1558 & 0.2743 & 0.57 & $1.415 \mathrm{E}+03$ & 0.3522 & 0.3382 & 1.04 & $4.212 \mathrm{E}+03$ & 0.0928 & 0.0828 & 1.12 \\
\hline $3.326 \mathrm{E}+02$ & 0.1677 & 0.2483 & 0.68 & $1.523 \mathrm{E}+03$ & 0.3177 & 0.3152 & 1.01 & $4.527 \mathrm{E}+03$ & 0.0825 & 0.0533 & 1.55 \\
\hline $3.575 \mathrm{E}+02$ & 0.1811 & 0.3250 & 0.56 & $1.638 \mathrm{E}+03$ & 0.3146 & 0.3353 & 0.94 & $4.869 \mathrm{E}+03$ & 0.0730 & 0.0480 & 1.52 \\
\hline $3.846 \mathrm{E}+02$ & 0.4134 & 0.2470 & 1.67 & $1.760 \mathrm{E}+03$ & 0.3074 & 0.2677 & 1.15 & $5.238 \mathrm{E}+03$ & 0.0581 & 0.0286 & 2.03 \\
\hline $4.139 \mathrm{E}+02$ & 0.4407 & 0.1976 & 2.23 & $1.893 \mathrm{E}+03$ & 0.2896 & 0.3137 & 0.92 & & & & \\
\hline $4.450 \mathrm{E}+02$ & 0.4759 & 0.2418 & 1.97 & $2.036 \mathrm{E}+03$ & 0.2626 & 0.2029 & 1.29 & & & & \\
\hline $4.783 E+02$ & 0.5148 & 0.2769 & 1.86 & $2.189 \mathrm{E}+03$ & 0.2516 & 0.1885 & 1.33 & & & & \\
\hline $5.144 \mathrm{E}+02$ & 0.3205 & 0.2613 & 1.23 & $2.354 \mathrm{E}+03$ & 0.2822 & 0.2302 & 1.23 & & & & \\
\hline $5.534 \mathrm{E}+02$ & 0.3433 & 0.3445 & 1.00 & & & & & & & & \\
\hline $5.952 \mathrm{E}+02$ & 0.3931 & 0.4537 & 0.87 & & & & & & & & \\
\hline $6.398 \mathrm{E}+02$ & 0.4244 & 0.4888 & 0.87 & & & & & & & & \\
\hline $6.878 \mathrm{E}+02$ & 0.4551 & 0.4290 & 1.06 & & & & & & & & \\
\hline $7.392 \mathrm{E}+02$ & 0.6693 & 0.3757 & 1.78 & & & & & & & & \\
\hline $7.951 E+02$ & 0.7144 & 0.3432 & 2.08 & & & & & & & BUG96/20 & \\
\hline
\end{tabular}


Figure 5.2-4: Detector 1 Data Comparison for $20 \mathrm{~cm}$ Sphere

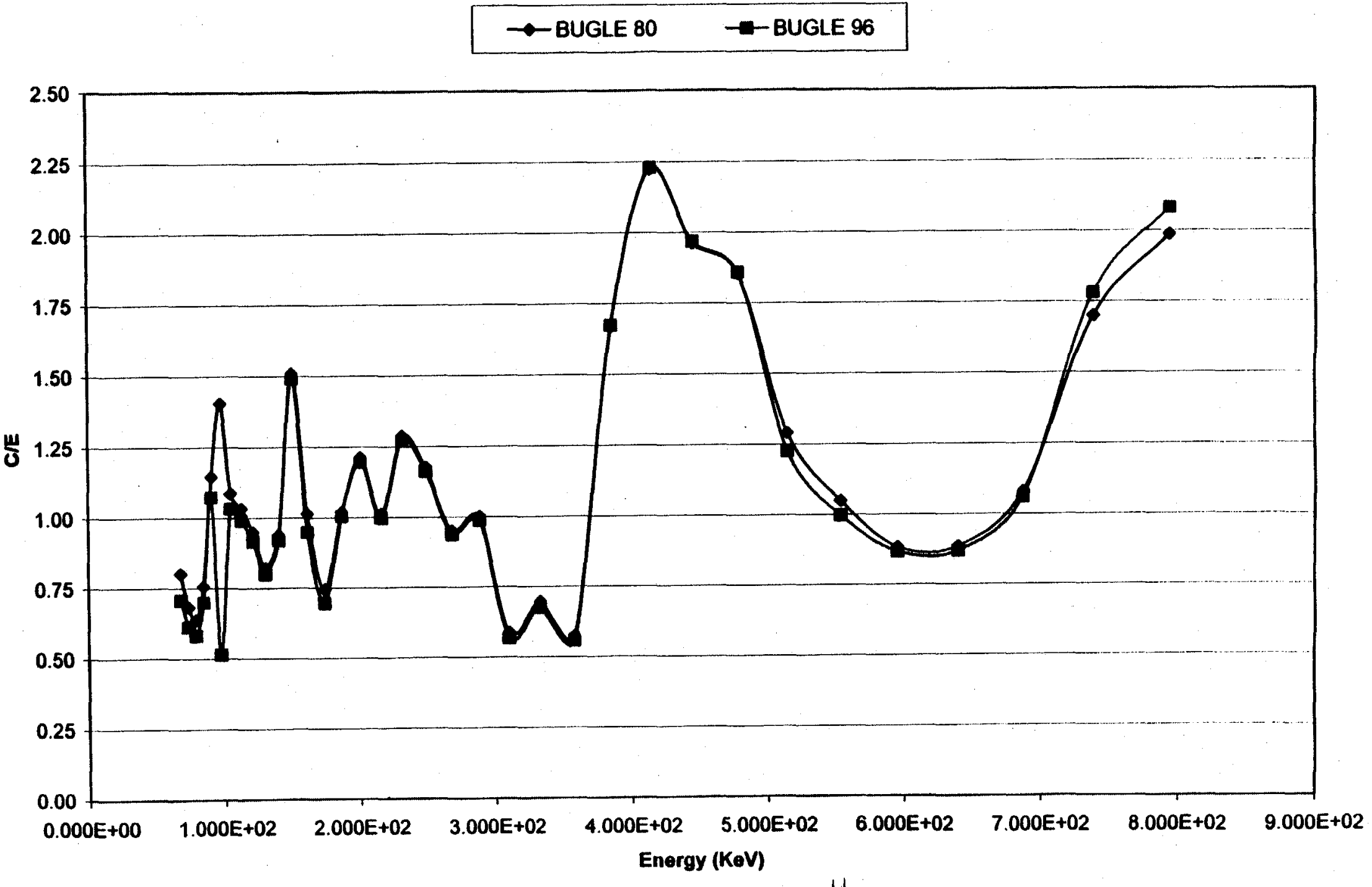

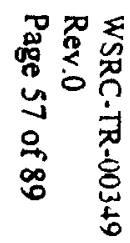




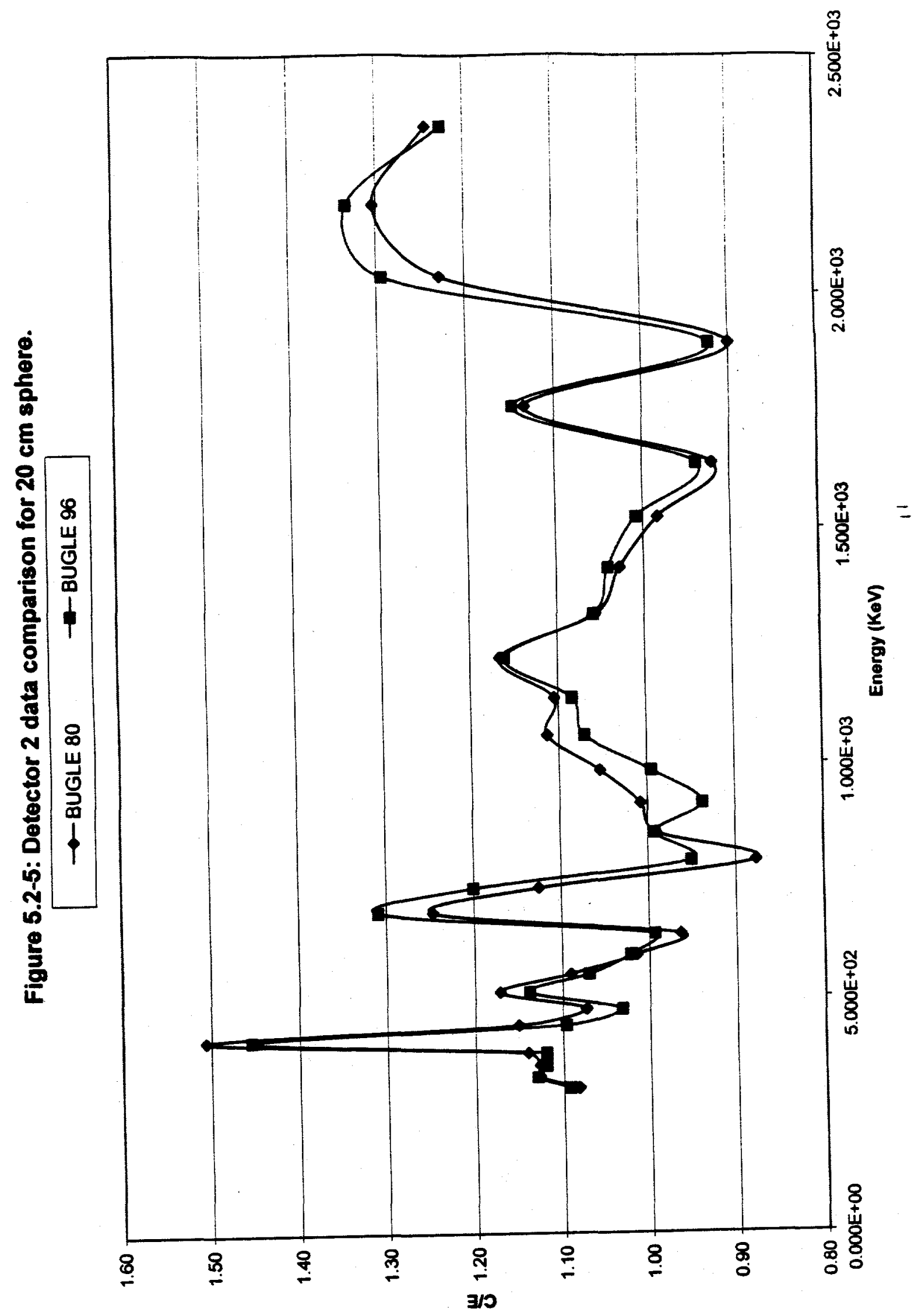


Figure 5.2-6: Detector 3 Data Comparison for $20 \mathrm{~cm}$ Sphere

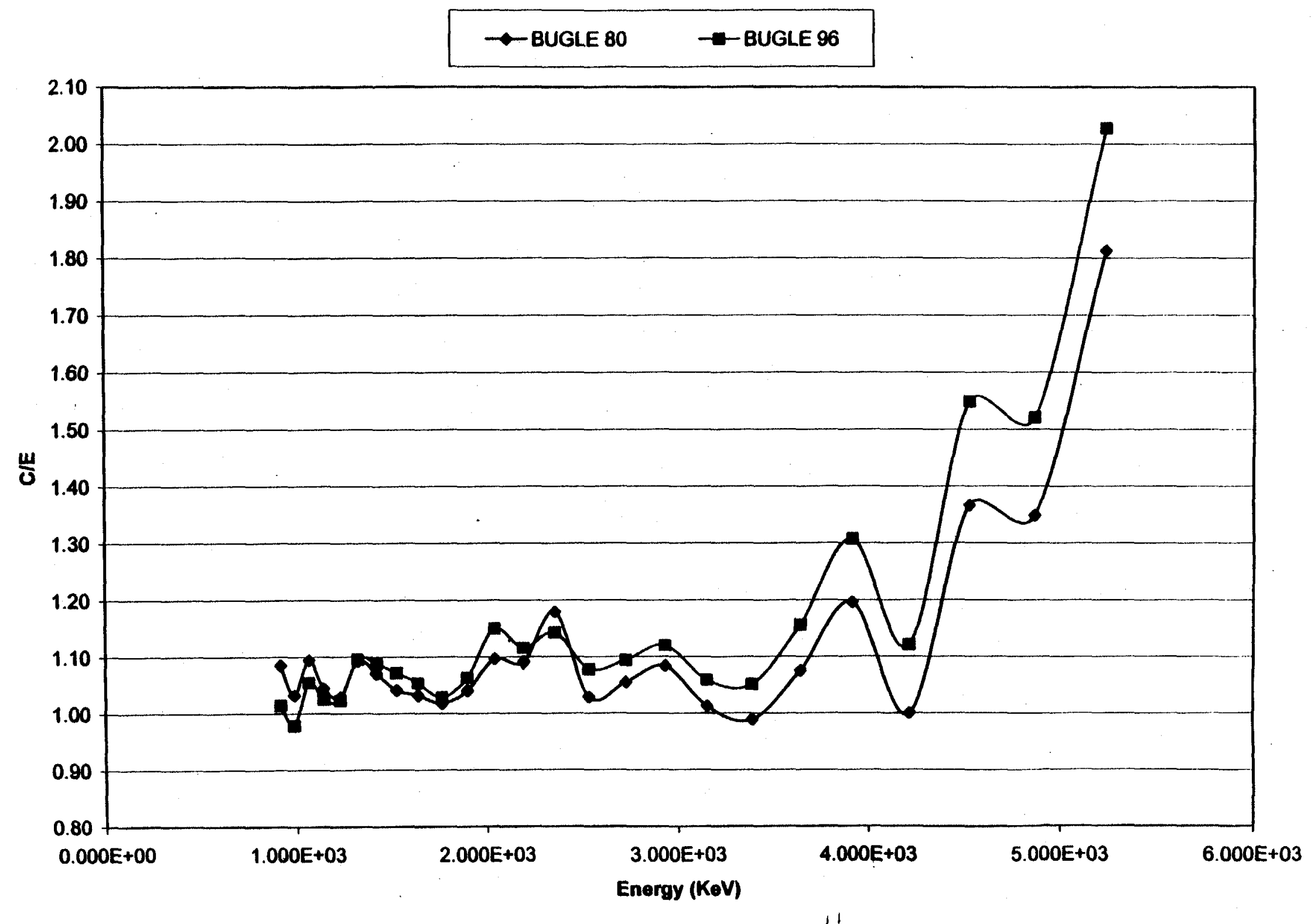


Table 5.2-5: Detector Measurements for 25cm Diameter Iron Sphere using BUGLE 80 cross section library.

\begin{tabular}{|c|c|c|c|c|c|c|c|c|c|c|c|}
\hline \multicolumn{4}{|c|}{ Detector 1} & \multicolumn{4}{|c|}{ Detector 2} & \multicolumn{4}{|c|}{ Detector 3} \\
\hline $\begin{array}{l}\text { Mid Energy } \\
(\mathrm{keV})\end{array}$ & $\begin{array}{l}\text { Calc. } \\
\text { (C) }\end{array}$ & $\begin{array}{c}\text { Exp. } \\
\text { (E) }\end{array}$ & $\mathrm{C} / \mathrm{E}$ & $\begin{array}{c}\text { Mid Energy } \\
(\mathrm{keV})\end{array}$ & $\begin{array}{l}\text { Calc. } \\
\text { (C) }\end{array}$ & $\begin{array}{l}\text { Exp. } \\
\text { (E) }\end{array}$ & $\mathrm{C} / \mathrm{E}$ & $\begin{array}{l}\text { Mid Energy } \\
(\mathrm{keV})\end{array}$ & $\begin{array}{c}\text { Calc. } \\
\text { (C) }\end{array}$ & $\begin{array}{l}\text { Exp. } \\
\text { (E) }\end{array}$ & $\mathrm{C} / \mathrm{E}$ \\
\hline $6.775 \mathrm{E}+01$ & 0.0368 & 0.0544 & 0.68 & $3.081 \mathrm{E}+02$ & 0.4547 & 0.3145 & 1.45 & $9.165 \mathrm{E}+02$ & 0.3804 & 0.3655 & 1.04 \\
\hline $7.285 \mathrm{E}+01$ & 0.0427 & 0.0541 & 0.79 & $3.317 \mathrm{E}+02$ & 0.4263 & 0.3202 & 1.33 & $9.870 \mathrm{E}+02$ & 0.3730 & 0.3674 & 1.02 \\
\hline $7.850 \mathrm{E}+01$ & 0.0496 & 0.0605 & 0.82 & $3.568 \mathrm{E}+02$ & 0.4062 & 0.3490 & 1.16 & $1.063 \mathrm{E}+03$ & 0.3755 & 0.3270 & 1.15 \\
\hline $8.415 \mathrm{E}+01$ & 0.0573 & 0.0538 & 1.07 & $3.833 \mathrm{E}+02$ & 0.3771 & 0.2886 & 1.31 & $1.142 \mathrm{E}+03$ & 0.3752 & 0.3694 & 1.02 \\
\hline $9.035 \mathrm{E}+01$ & 0.0663 & 0.0557 & 1.19 & $4.110 \mathrm{E}+02$ & 0.3390 & 0.2140 & 1.58 & $1.226 \mathrm{E}+03$ & 0.3624 & 0.3578 & 1.01 \\
\hline $9.710 \mathrm{E}+01$ & 0.0725 & 0.0427 & 1.70 & $4.423 \mathrm{E}+02$ & 0.3071 & 0.2398 & 1.28 & $1.320 \mathrm{E}+03$ & 0.3329 & 0.3001 & 1.11 \\
\hline $1.044 \mathrm{E}+02$ & 0.0801 & 0.0575 & 1.39 & $4.762 E+02$ & 0.3326 & 0.3231 & 1.03 & $1.419 \mathrm{E}+03$ & 0.2940 & 0.2789 & 1.05 \\
\hline $1.123 \mathrm{E}+02$ & 0.0887 & 0.0748 & 1.19 & $5.130 \mathrm{E}+02$ & 0.3601 & 0.3073 & 1.17 & $1.524 \mathrm{E}+03$ & 0.2545 & 0.2539 & 1.00 \\
\hline $1.208 \mathrm{E}+02$ & 0.0983 & 0.0919 & 1.07 & $5.514 \mathrm{E}+02$ & 0.3900 & 0.3561 & 1.10 & $1.638 \mathrm{E}+03$ & 0.2531 & 0.2482 & 1.02 \\
\hline $1.299 \mathrm{E}+02$ & 0.1090 & 0.1262 & 0.86 & $5.927 \mathrm{E}+02$ & 0.4569 & 0.4423 & 1.03 & $1.761 \mathrm{E}+03$ & 0.2485 & 0.2501 & 0.99 \\
\hline $1.395 \mathrm{E}+02$ & 0.1209 & 0.1448 & 0.83 & $6.369 E+02$ & 0.5359 & 0.5874 & 0.91 & $1.896 \mathrm{E}+03$ & 0.2259 & 0.2193 & 1.03 \\
\hline $1.496 \mathrm{E}+02$ & 0.1342 & 0.0941 & 1.43 & $6.840 \mathrm{E}+02$ & 0.5865 & 0.4667 & 1.26 & $2.039 \mathrm{E}+03$ & 0.1922 & 0.1797 & 1.07 \\
\hline $1.609 \mathrm{E}+02$ & 0.1225 & 0.0911 & 1.35 & $7.356 \mathrm{E}+02$ & 0.4271 & 0.3461 & 1.23 & $2.191 \mathrm{E}+03$ & 0.1904 & 0.1737 & 1.10 \\
\hline $1.734 E+02$ & 0.1273 & 0.1630 & 0.78 & $7.917 \mathrm{E}+02$ & 0.2679 & 0.3346 & 0.80 & $2.355 \mathrm{E}+03$ & 0.2016 & 0.1622 & 1.24 \\
\hline $1.863 \mathrm{E}+02$ & 0.1731 & 0.1657 & 1.04 & $8.521 \mathrm{E}+02$ & 0.3153 & 0.3418 & 0.92 & $2.533 \mathrm{E}+03$ & 0.1420 & 0.1373 & 1.03 \\
\hline $2.004 E+02$ & 0.1855 & 0.1383 & 1.34 & $9.155 \mathrm{E}+02$ & 0.3673 & 0.3604 & 1.02 & $2.726 \mathrm{E}+03$ & 0.1285 & 0.1254 & 1.03 \\
\hline $2.157 \mathrm{E}+02$ & 0.1945 & 0.1552 & 1.25 & $9.850 \mathrm{E}+02$ & 0.3733 & 0.3533 & 1.06 & $2.931 \mathrm{E}+03$ & 0.1161 & 0.1098 & 1.06 \\
\hline $2.315 \mathrm{E}+02$ & 0.2127 & 0.1591 & 1.34 & $1.060 \mathrm{E}+03$ & 0.3762 & 0.3245 & 1.16 & $3.150 \mathrm{E}+03$ & 0.0991 & 0.0976 & 1.02 \\
\hline $2.484 E+02$ & 0.2359 & 0.1670 & 1.41 & $1.139 \mathrm{E}+03$ & 0.3762 & 0.3691 & 1.02 & $3.387 \mathrm{E}+03$ & 0.0800 & 0.0839 & 0.95 \\
\hline $2.677 \mathrm{E}+02$ & 0.2438 & 0.2204 & 1.11 & $1.225 \mathrm{E}+03$ & 0.3629 & 0.3533 & 1.03 & $3.641 \mathrm{E}+03$ & 0.0744 & 0.0723 & 1.03 \\
\hline $2.880 \mathrm{E}+02$ & 0.2632 & 0.2387 & 1.10 & $1.316 \mathrm{E}+03$ & 0.3357 & 0.3202 & 1.05 & $3.916 \mathrm{E}+03$ & 0.0666 & 0.0623 & 1.07 \\
\hline $3.095 \mathrm{E}+02$ & 0.1751 & 0.3000 & 0.58 & $1.415 \mathrm{E}+03$ & 0.2973 & 0.2743 & 1.08 & $4.212 \mathrm{E}+03$ & 0.0559 & 0.0545 & 1.03 \\
\hline $3.326 \mathrm{E}+02$ & 0.1884 & 0.3039 & 0.62 & $1.523 \mathrm{E}+03$ & 0.2554 & 0.2484 & 1.03 & $4.527 \mathrm{E}+03$ & 0.0487 & 0.0412 & 1.18 \\
\hline $3.575 E+02$ & 0.2036 & 0.3457 & 0.59 & $1.638 \mathrm{E}+03$ & 0.2537 & 0.2743 & 0.92 & $4.869 \mathrm{E}+03$ & 0.0432 & 0.0360 & 1.20 \\
\hline $3.846 \mathrm{E}+02$ & 0.5109 & 0.2909 & 1.76 & $1.760 \mathrm{E}+03$ & 0.2489 & 0.2369 & 1.05 & $5.238 \mathrm{E}+03$ & 0.0345 & 0.0239 & 1.45 \\
\hline $4.139 \mathrm{E}+02$ & 0.5446 & 0.1904 & 2.86 & $1.893 \mathrm{E}+03$ & 0.2266 & 0.2355 & 0.96 & & & & \\
\hline $4.450 \mathrm{E}+02$ & 0.5882 & 0.2439 & 2.41 & $2.036 \mathrm{E}+03$ & 0.1929 & 0.1795 & 1.07 & & & & \\
\hline $4.783 E+02$ & 0.6363 & 0.2961 & 2.15 & $2.189 \mathrm{E}+03$ & 0.1905 & 0.1565 & 1.22 & & & & \\
\hline $5.144 \mathrm{E}+02$ & 0.3704 & 0.2805 & 1.32 & $2.354 \mathrm{E}+03$ & 0.2265 & 0.1317 & 1.72 & & & & \\
\hline $5.534 E+02$ & 0.3968 & 0.3470 & 1.14 & & & & & & & & \\
\hline $5.952 \mathrm{E}+02$ & 0.4346 & 0.4840 & 0.90 & & & & & & & & \\
\hline $6.398 \mathrm{E}+02$ & 0.4693 & 0.5636 & 0.83 & & & & & & & & \\
\hline $6.878 \mathrm{E}+02$ & 0.5032 & 0.4540 & 1.11 & & & & & & & & \\
\hline $7.392 \mathrm{E}+02$ & 0.6958 & 0.3809 & 1.83 & & & & & & & & \\
\hline $7.951 \mathrm{E}+02$ & 0.7428 & 0.3561 & 2.09 & & & & & & & BUG80/25 & \\
\hline
\end{tabular}


J

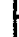

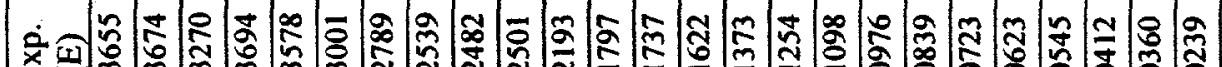 齐 $m$ 妾

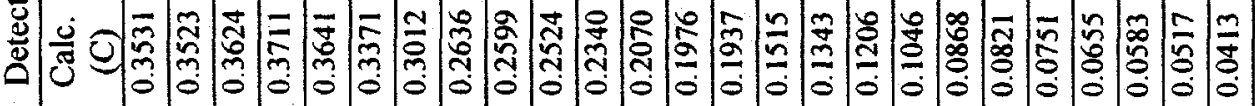

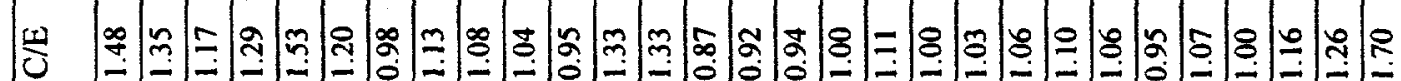

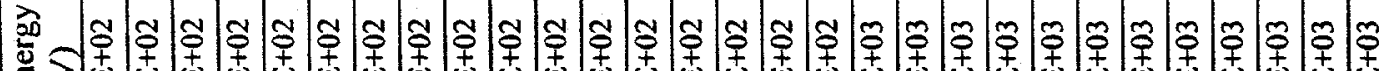

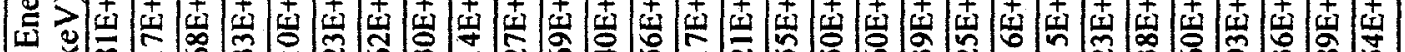

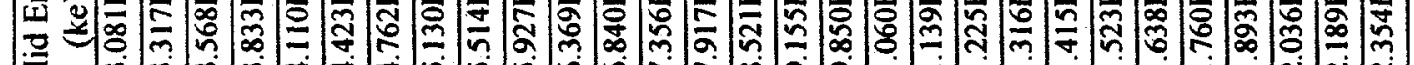
$\Sigma$

Un

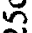

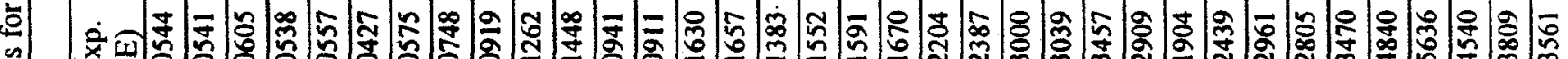
E)

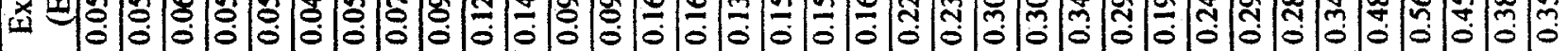

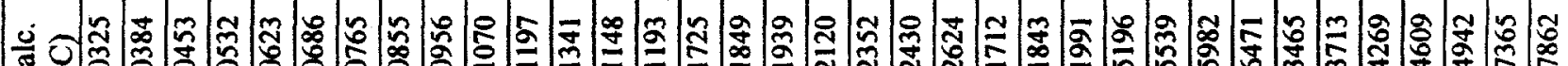

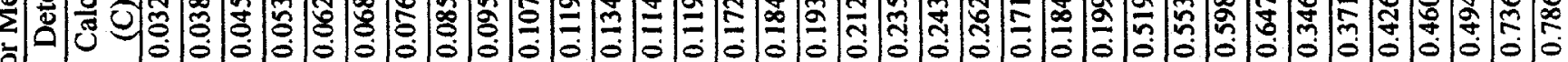

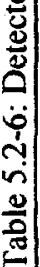

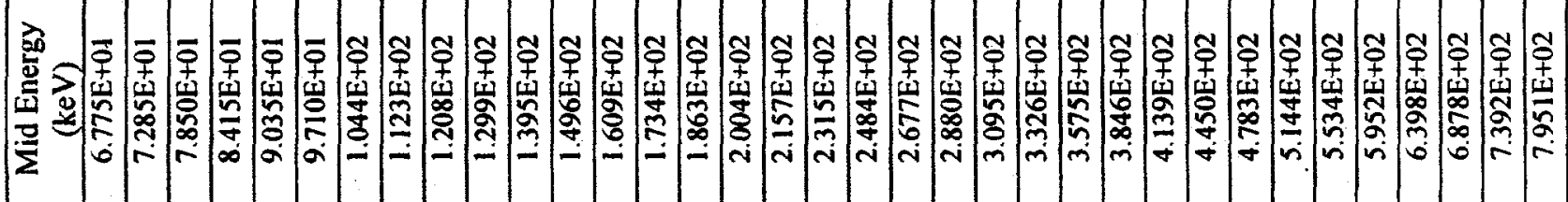


Figure 5.2-7: Detector 1 Data Comparison for $25 \mathrm{~cm}$ Sphere

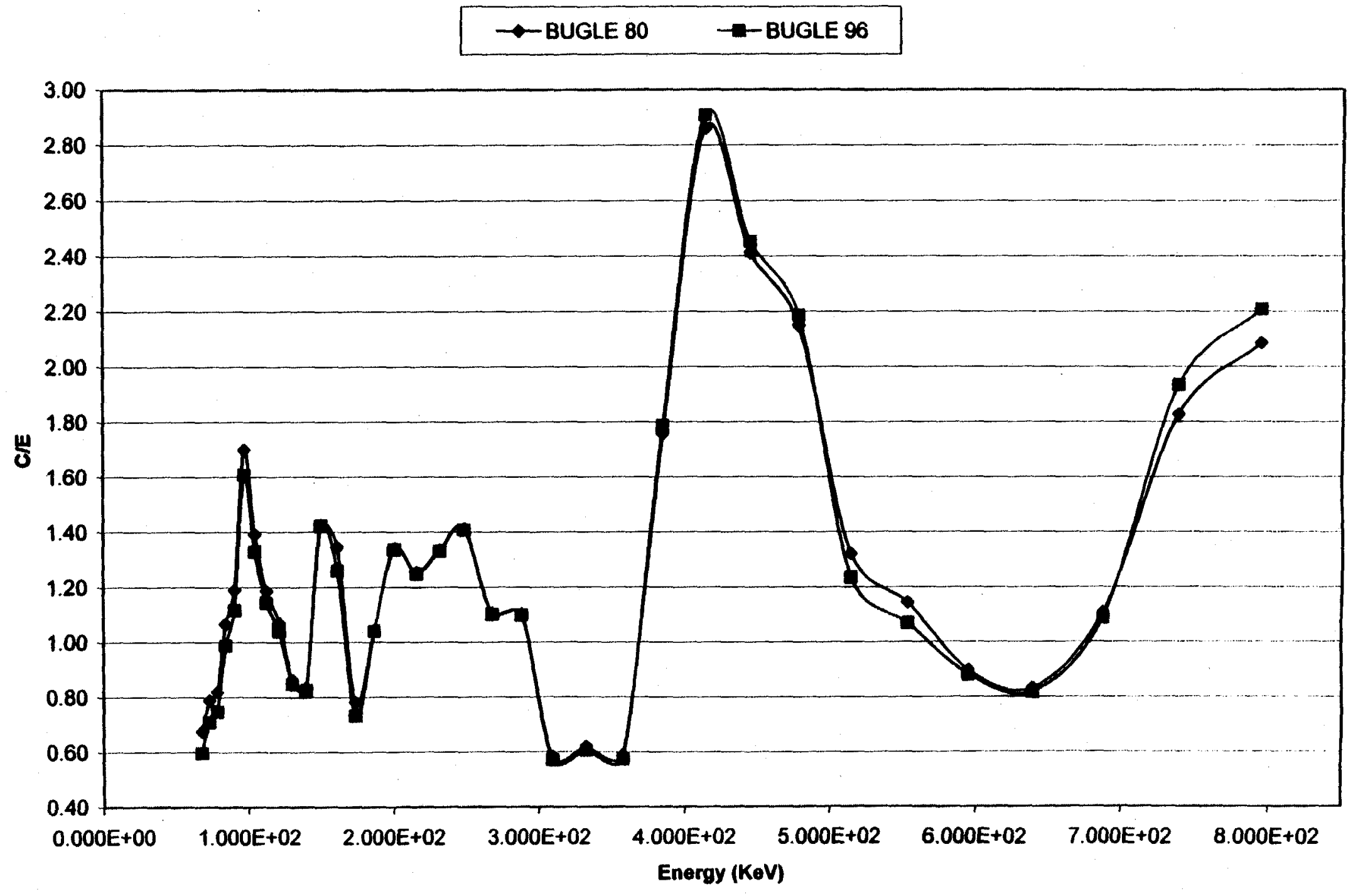


Figure 5.2-8: Detector 2 data comparison for $25 \mathrm{~cm}$ sphere.

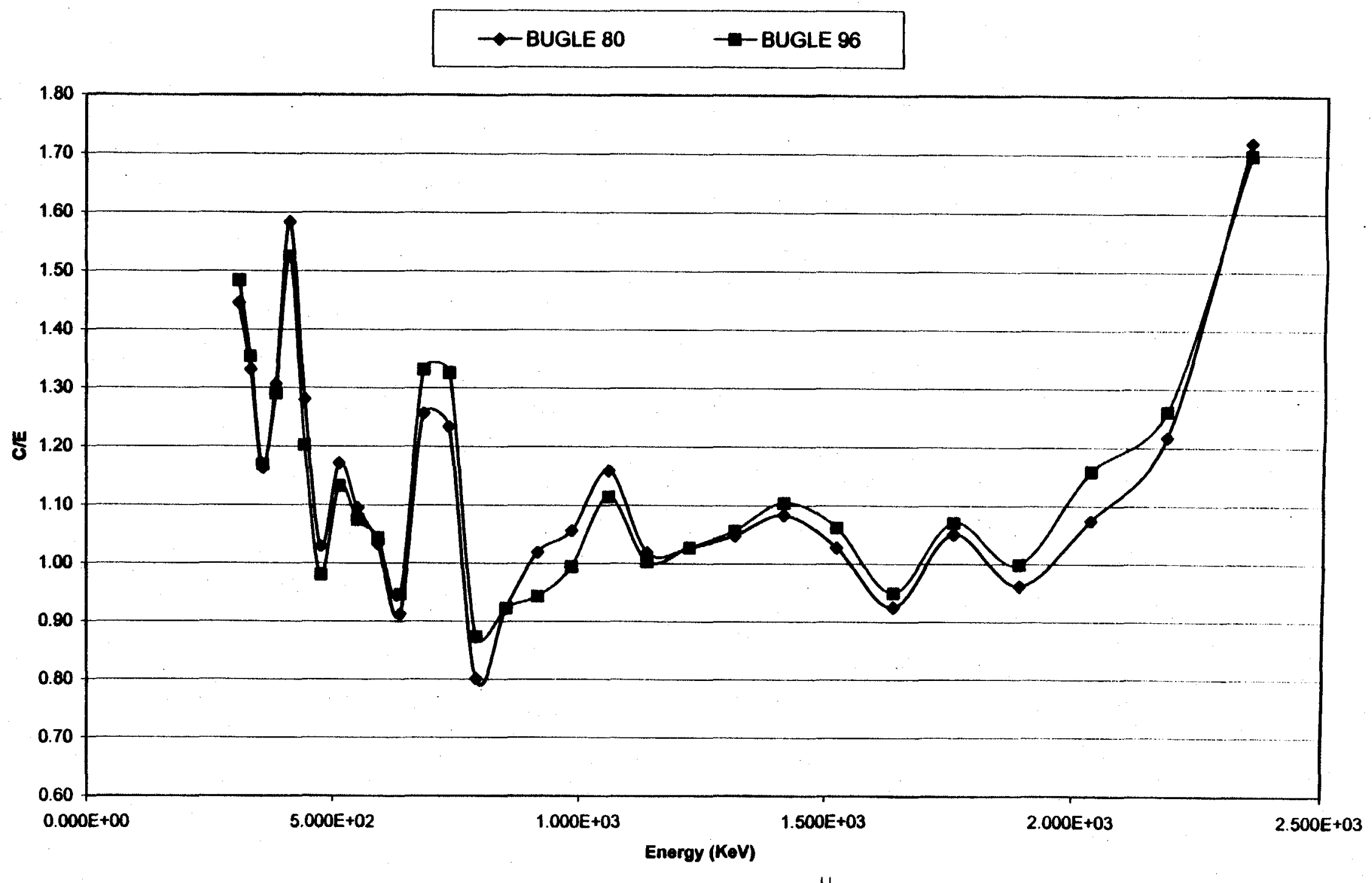


Figure 5.2-9: Detector 3 Data Comparison for $25 \mathrm{~cm}$ Sphere

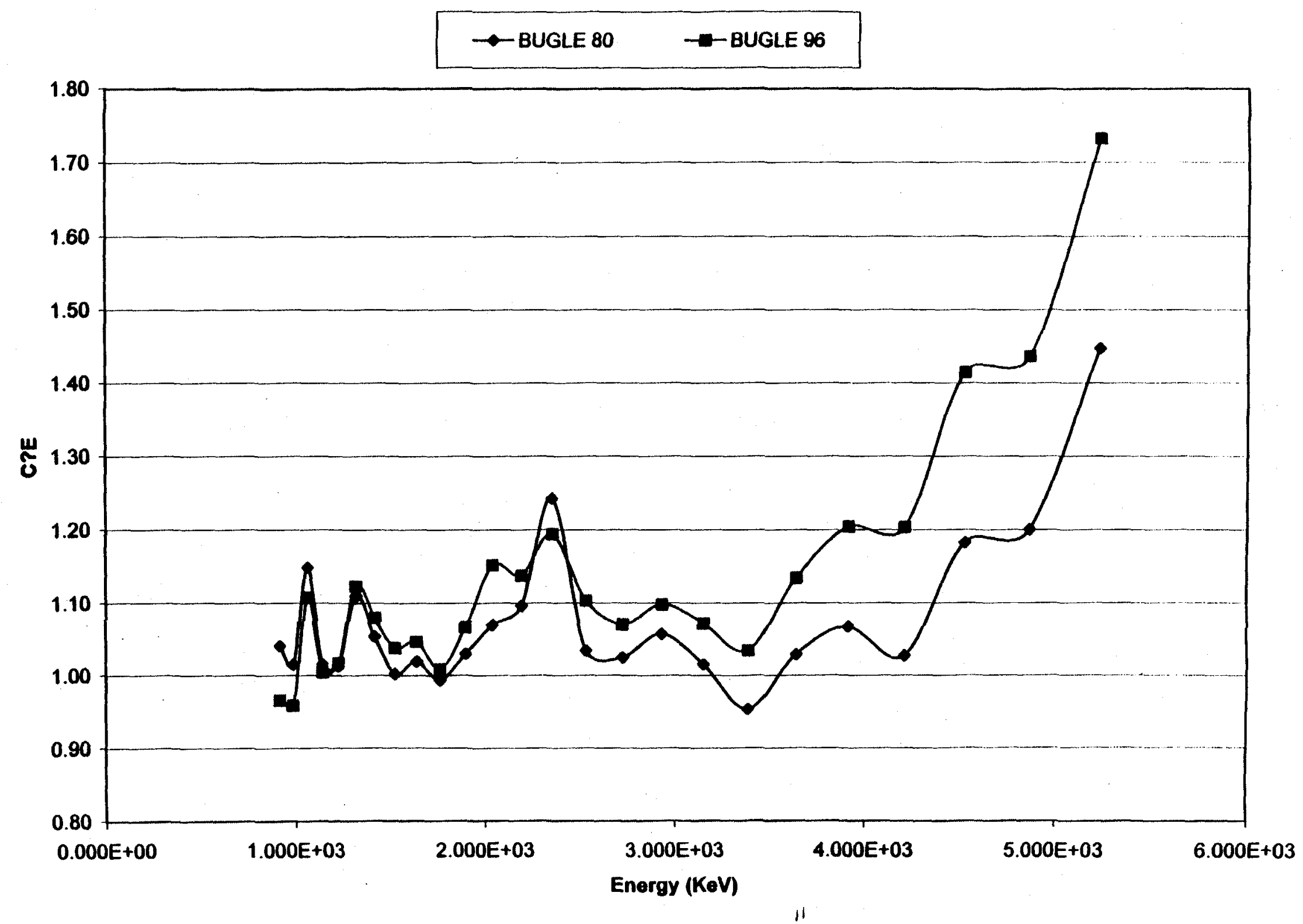


Table 5.2-7: Detector Measurements for 30cm Diameter Iron Sphere using BUGLE 80 cross section library.

\begin{tabular}{|c|c|c|c|c|c|c|c|c|c|c|c|}
\hline \multicolumn{4}{|c|}{ Detector 1} & \multicolumn{4}{|c|}{ Detector 2} & \multicolumn{4}{|c|}{ Detector 3} \\
\hline $\begin{array}{l}\text { Mid Energy } \\
\text { (keV) }\end{array}$ & $\begin{array}{l}\text { Calc. } \\
\text { (C) }\end{array}$ & $\begin{array}{l}\text { Exp. } \\
\text { (E) }\end{array}$ & $\mathrm{C} / \mathrm{E}$ & $\begin{array}{c}\text { Mid Energy } \\
(\mathrm{keV})\end{array}$ & $\begin{array}{l}\text { Calc. } \\
\text { (C) }\end{array}$ & $\begin{array}{c}\text { Exp. } \\
\text { (E) }\end{array}$ & $\mathrm{C} / \mathrm{E}$ & $\begin{array}{l}\text { Mid Energy } \\
(\mathrm{keV})\end{array}$ & $\begin{array}{l}\text { Calc. } \\
\text { (C) }\end{array}$ & $\begin{array}{c}\text { Exp. } \\
\text { (E) }\end{array}$ & $\mathrm{C} / \mathrm{E}$ \\
\hline $6.775 \mathrm{E}+01$ & 0.0465 & 0.0303 & 1.53 & $3.081 \mathrm{E}+02$ & 0.5434 & 0.3572 & 1.52 & $9.165 \mathrm{E}+02$ & 0.3541 & 0.3682 & 0.96 \\
\hline $7.285 \mathrm{E}+01$ & 0.0539 & 0.0357 & 1.51 & $3.317 \mathrm{E}+02$ & 0.5035 & 0.3445 & 1.46 & $9.870 \mathrm{E}+02$ & 0.3427 & 0.3703 & 0.93 \\
\hline $7.850 \mathrm{E}+01$ & 0.0625 & 0.0510 & 1.23 & $3.568 \mathrm{E}+02$ & 0.4723 & 0.3728 & 1.27 & $1.063 \mathrm{E}+03$ & 0.3396 & 0.3114 & 1.09 \\
\hline $8.415 \mathrm{E}+01$ & 0.0723 & 0.0515 & 1.40 & $3.833 \mathrm{E}+02$ & 0.4288 & 0.2693 & 1.59 & $1.142 E+03$ & 0.3327 & 0.3430 & 0.97 \\
\hline $9.035 \mathrm{E}+01$ & 0.0835 & 0.0603 & 1.39 & $4.110 \mathrm{E}+02$ & 0.3730 & 0.2084 & 1.79 & $1.226 \mathrm{E}+03$ & 0.3161 & 0.3367 & 0.94 \\
\hline $9.710 \mathrm{E}+01$ & 0.0905 & 0.0502 & 1.80 & $4.423 \mathrm{E}+02$ & 0.3247 & 0.2623 & 1.24 & $1.320 \mathrm{E}+03$ & 0.2849 & 0.2693 & 1.06 \\
\hline $1.044 \mathrm{E}+02$ & 0.0993 & 0.0511 & 1.94 & $4.762 \mathrm{E}+02$ & 0.3500 & 0.3289 & 1.06 & $1.419 \mathrm{E}+03$ & 0.2444 & 0.2399 & 1.02 \\
\hline $1.123 \mathrm{E}+02$ & 0.1091 & 0.0857 & 1.27 & $5.130 \mathrm{E}+02$ & 0.3771 & 0.3019 & 1.25 & $1.524 \mathrm{E}+03$ & 0.2034 & 0.2104 & 0.97 \\
\hline $1.208 \mathrm{E}+02$ & 0.1199 & 0.0861 & 1.39 & $5.514 \mathrm{E}+02$ & 0.4063 & 0.3799 & 1.07 & $1.638 \mathrm{E}+03$ & 0.2021 & 0.1986 & 1.02 \\
\hline $1.299 \mathrm{E}+02$ & 0.1320 & 0.1159 & 1.14 & $5.927 \mathrm{E}+02$ & 0.4754 & 0.5019 & 0.95 & $1.761 \mathrm{E}+03$ & 0.1984 & 0.2056 & 0.96 \\
\hline $1.395 E+02$ & 0.1451 & 0.1567 & 0.93 & $6.369 \mathrm{E}+02$ & 0.5572 & 0.6110 & 0.91 & $1.896 \mathrm{E}+03$ & 0.1770 & 0.1742 & 1.02 \\
\hline $1.496 \mathrm{E}+02$ & 0.1599 & 0.1382 & 1.16 & $6.840 \mathrm{E}+02$ & 0.6076 & 0.4225 & 1.44 & $2.039 \mathrm{E}+03$ & 0.1455 & 0.1348 & 1.08 \\
\hline $1.609 \mathrm{E}+02$ & 0.1543 & 0.1170 & 1.32 & $7.356 \mathrm{E}+02$ & 0.4243 & 0.3516 & 1.21 & $2.191 \mathrm{E}+03$ & 0.1434 & 0.1302 & 1.10 \\
\hline $1.734 \mathrm{E}+02$ & 0.1603 & 0.1567 & 1.02 & $7.917 \mathrm{E}+02$ & 0.2404 & 0.3204 & 0.75 & $2.355 \mathrm{E}+03$ & 0.1528 & 0.1256 & 1.22 \\
\hline $1.863 \mathrm{E}+02$ & 0.2066 & 0.2075 & 1.00 & $8.521 E+02$ & 0.2888 & 0.3204 & 0.90 & $2.533 \mathrm{E}+03$ & 0.1023 & 0.0989 & 1.03 \\
\hline $2.004 \mathrm{E}+02$ & 0.2214 & 0.1829 & 1.21 & $9.155 \mathrm{E}+02$ & 0.3419 & 0.3558 & 0.96 & $2.726 \mathrm{E}+03$ & 0.0913 & 0.0890 & 1.03 \\
\hline $2.157 \mathrm{E}+02$ & 0.2322 & 0.1742 & 1.33 & $9.850 \mathrm{E}+02$ & 0.3432 & 0.3615 & 0.95 & $2.931 \mathrm{E}+03$ & 0.0817 & 0.0806 & 1.01 \\
\hline $2.315 \mathrm{E}+02$ & 0.2538 & 0.1974 & 1.29 & $1.060 \mathrm{E}+03$ & 0.3407 & 0.2864 & 1.19 & $3.150 \mathrm{E}+03$ & 0.0692 & 0.0680 & 1.02 \\
\hline $2.484 E+02$ & 0.2816 & 0.1974 & 1.43 & $1.139 \mathrm{E}+03$ & 0.3343 & 0.3317 & 1.01 & $3.387 \mathrm{E}+03$ & 0.0552 & 0.0587 & 0.94 \\
\hline $2.677 \mathrm{E}+02$ & 0.2910 & 0.2613 & 1.11 & $1.225 \mathrm{E}+03$ & 0.3167 & 0.2991 & 1.06 & $3.641 \mathrm{E}+03$ & 0.0511 & 0.0484 & 1.06 \\
\hline $2.880 \mathrm{E}+02$ & 0.3142 & 0.3121 & 1.01 & $1.316 \mathrm{E}+03$ & 0.2876 & 0.2778 & 1.04 & $3.916 \mathrm{E}+03$ & 0.0454 & 0.0398 & 1.14 \\
\hline $3.095 \mathrm{E}+02$ & 0.1876 & 0.3832 & 0.49 & $1.415 \mathrm{E}+03$ & 0.2473 & 0.2013 & 1.23 & $4.212 E+03$ & 0.0377 & 0.0341 & 1.11 \\
\hline $3.326 \mathrm{E}+02$ & 0.2019 & 0.3484 & 0.58 & $1.523 \mathrm{E}+03$ & 0.2040 & 0.2282 & 0.89 & $4.527 \mathrm{E}+03$ & 0.0328 & 0.0286 & 1.15 \\
\hline $3.575 \mathrm{E}+02$ & 0.2181 & 0.3788 & 0.58 & $1.638 \mathrm{E}+03$ & 0.2026 & 0.2041 & 0.99 & $4.869 E+03$ & 0.0292 & 0.0213 & 1.38 \\
\hline $3.846 \mathrm{E}+02$ & 0.6030 & 0.3237 & 1.86 & $1.760 \mathrm{E}+03$ & 0.1987 & 0.1999 & 0.99 & $5.238 \mathrm{E}+03$ & 0.0236 & 0.0129 & 1.83 \\
\hline $4.139 \mathrm{E}+02$ & 0.6428 & 0.1959 & 3.28 & $1.893 \mathrm{E}+03$ & 0.1781 & 0.1531 & 1.16 & & & & \\
\hline $4.450 \mathrm{E}+02$ & 0.6943 & 0.2279 & 3.05 & $2.036 \mathrm{E}+03$ & 0.1466 & 0.1355 & 1.08 & & & & \\
\hline $4.783 \mathrm{E}+02$ & 0.7510 & 0.2845 & 2.64 & $2.189 \mathrm{E}+03$ & 0.1430 & 0.1273 & 1.12 & & & & \\
\hline $5.144 \mathrm{E}+02$ & 0.3921 & 0.3077 & 1.27 & $2.354 \mathrm{E}+03$ & 0.1732 & 0.0967 & 1.79 & & & & \\
\hline $5.534 \mathrm{E}+02$ & 0.4201 & 0.3788 & 1.11 & & & & & & & & \\
\hline $5.952 \mathrm{E}+02$ & 0.4527 & 0.5313 & 0.85 & & & & & & & & \\
\hline $6.398 \mathrm{E}+02$ & 0.4888 & 0.5995 & 0.82 & & & & & & & & \\
\hline $6.878 \mathrm{E}+02$ & 0.5241 & 0.4674 & 1.12 & & & & & & & & \\
\hline $7.392 \mathrm{E}+02$ & 0.7237 & 0.3527 & 2.05 & & & & & & & & \\
\hline $7.951 \mathrm{E}+02$ & 0.7725 & 0.3527 & 2.19 & & & & & & & BUG80/30 & \\
\hline
\end{tabular}


BUGLE 96 VALIDATION WITH MORSE USING WATER

WSRC-TR-00349

AND IRON EXPERIMENTAL DATA FROM SINDBAD 97

Rev.0

Page 66 of 89

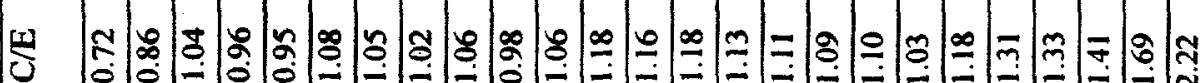

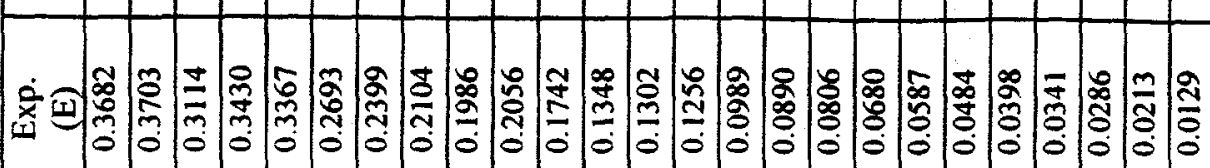
m

站

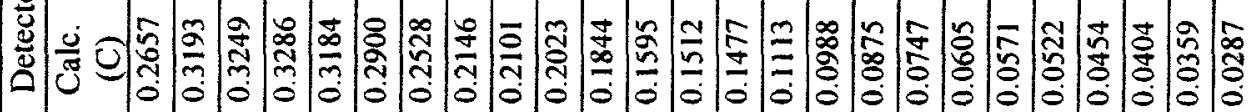

.

के

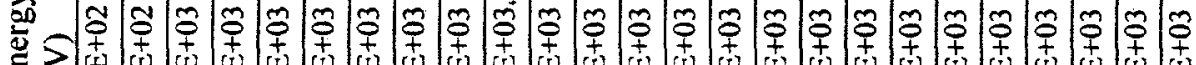

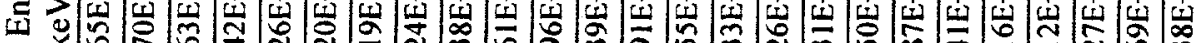

焉

U

홀

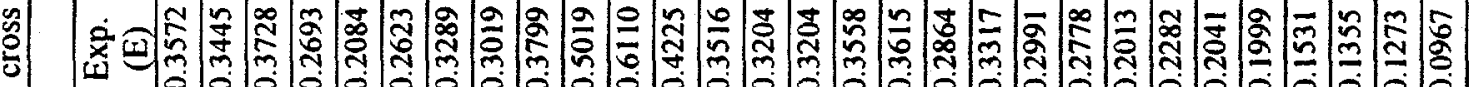

ฉำ

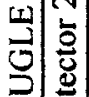

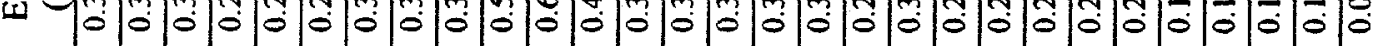

(n)

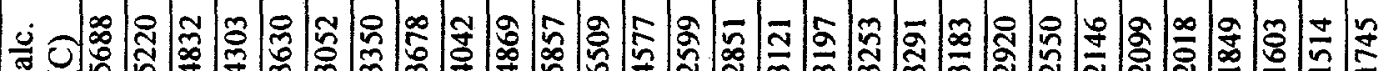

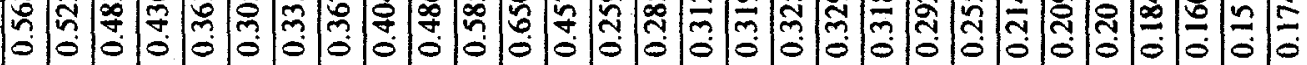

(

$\frac{\text { के }}{\frac{0}{2}}$

톨

क्षे

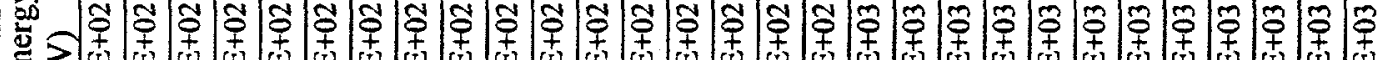

जี ق

承

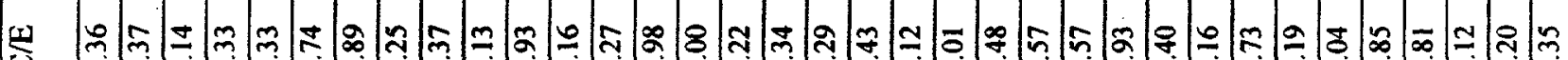

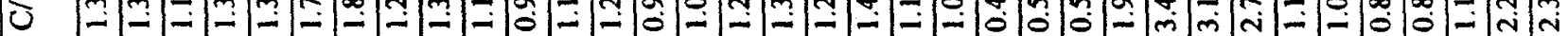

ह

홍

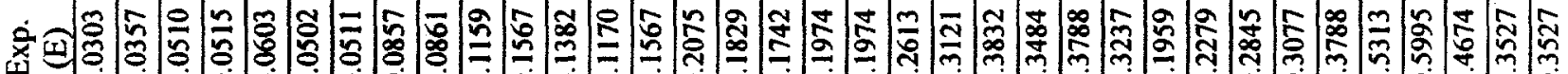
-

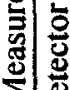

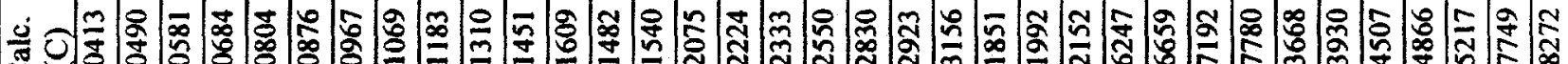
(1)

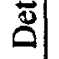

$\ddot{\dot{a}}$ 忽总

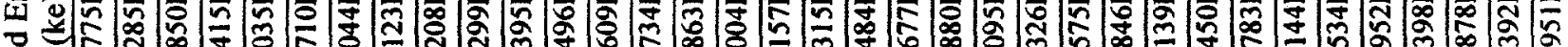

竞 $\sum$ 
Figure 5.2-10: Detector 1 Data Comparison for $30 \mathrm{~cm}$ Sphere

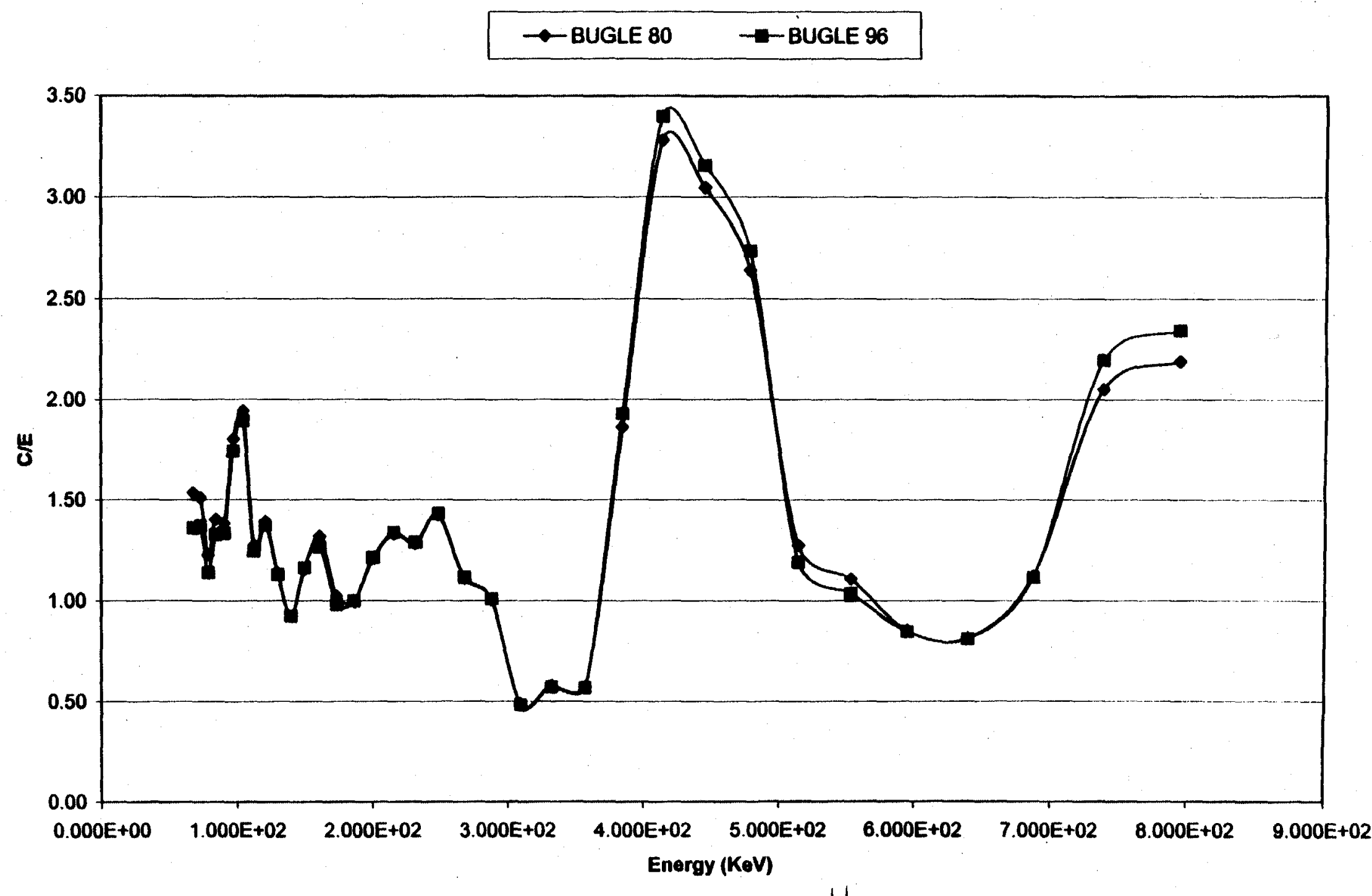




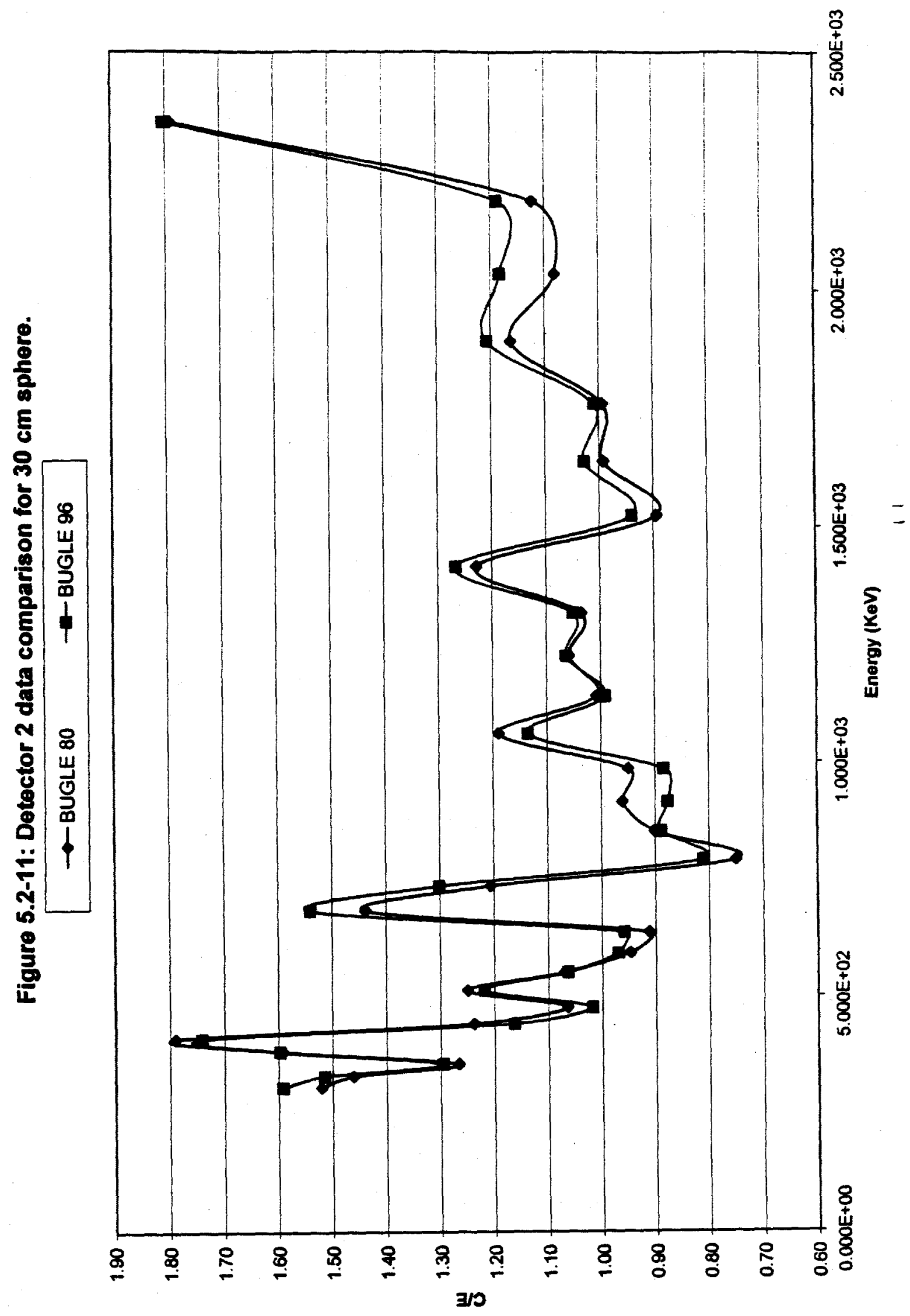


Figure 5.2-12: Detector 3 Data Comparison for $30 \mathrm{~cm}$ Sphere

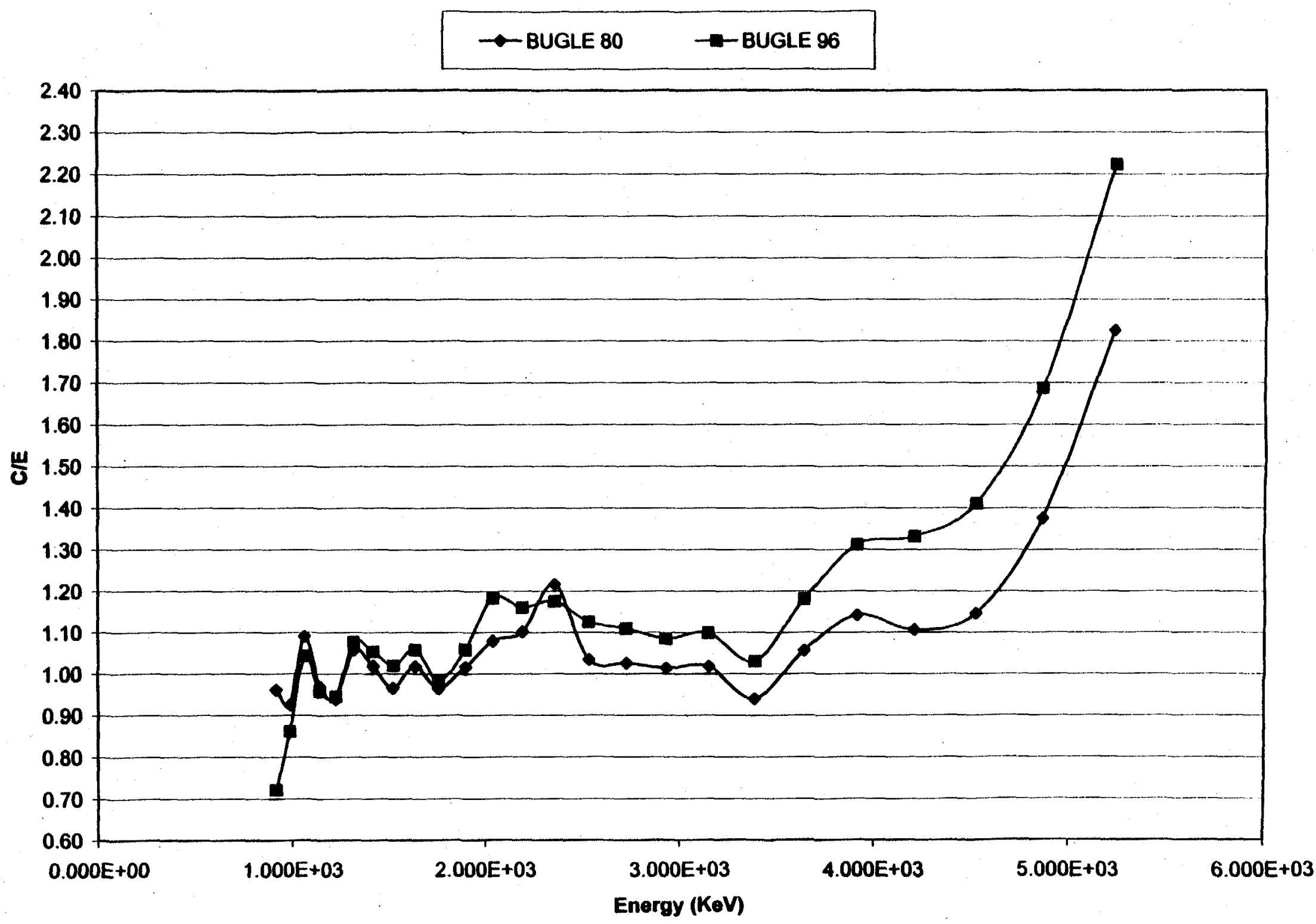


虫

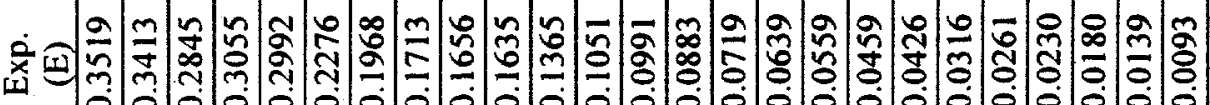

范

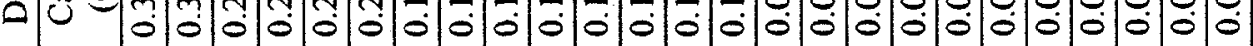

旁

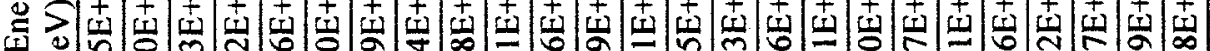

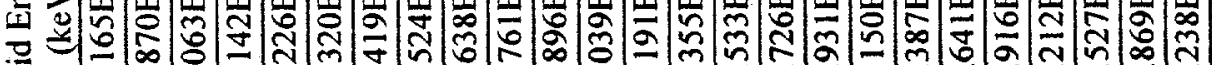
$\Sigma$

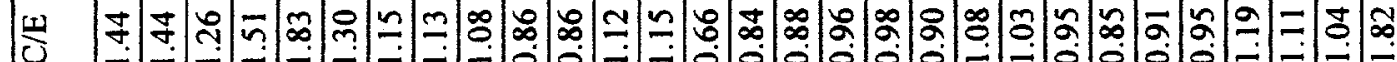

숭

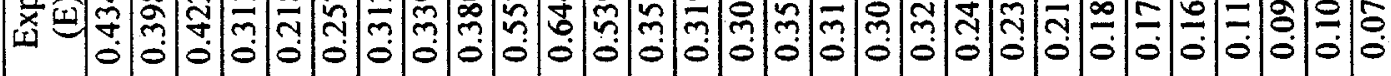
N

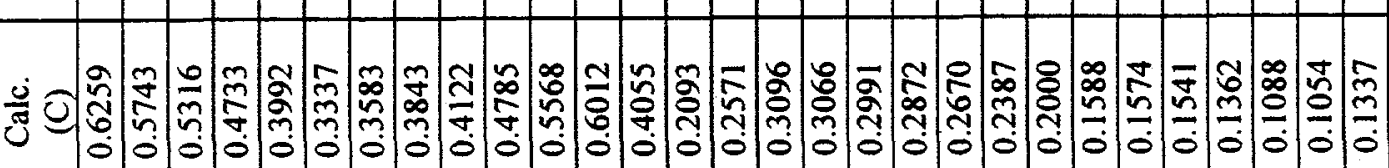

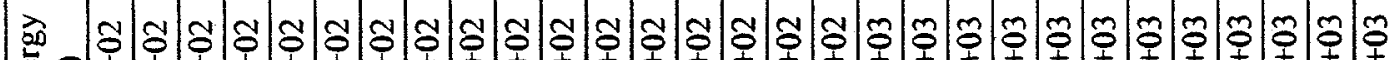

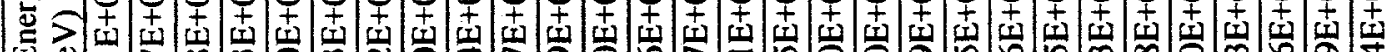

車 包

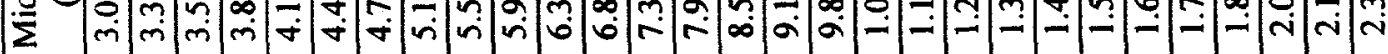

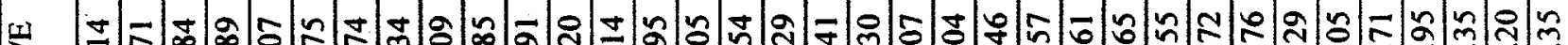

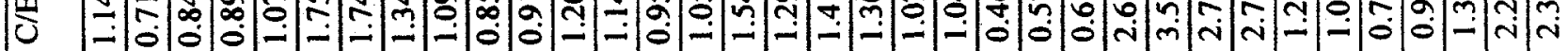

ֻ

호

$\stackrel{3}{3}$ 政

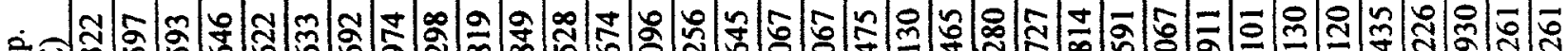
х

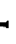

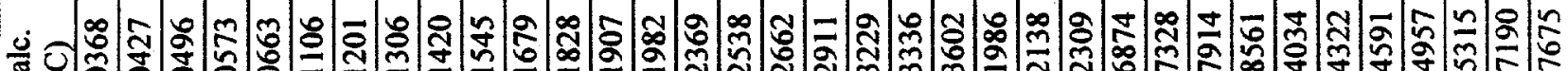

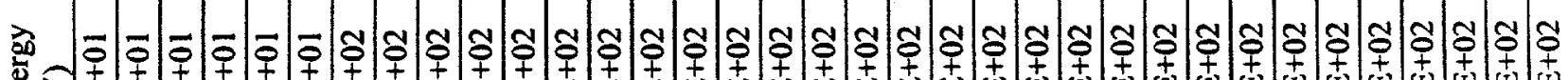
⿹勹巳

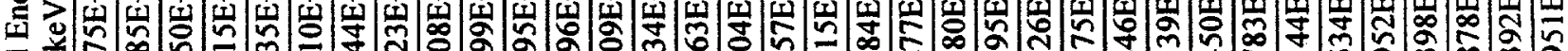
马 $\sum$ 
Table 5.2-10: Detector Measurements for 35cm Diameter Iron Sphere using BUGLE 96 cross section library.

\begin{tabular}{|c|c|c|c|c|c|c|c|c|c|c|c|}
\hline \multicolumn{4}{|c|}{ Detector 1} & \multicolumn{4}{|c|}{ Detector 2} & \multicolumn{4}{|c|}{ Detector 3} \\
\hline $\begin{array}{c}\text { Mid Energy } \\
(\mathrm{keV})\end{array}$ & $\begin{array}{l}\text { Calc. } \\
\text { (C) }\end{array}$ & $\begin{array}{c}\text { Exp. } \\
\text { (E) }\end{array}$ & $\mathrm{C} / \mathrm{E}$ & $\begin{array}{c}\text { Mid Energy } \\
(\mathrm{keV})\end{array}$ & $\begin{array}{l}\text { Calc. } \\
\text { (C) }\end{array}$ & $\begin{array}{l}\text { Exp. } \\
\text { (E) }\end{array}$ & $\mathrm{C} / \mathrm{E}$ & $\begin{array}{c}\text { Mid Energy } \\
(\mathrm{keV})\end{array}$ & $\begin{array}{l}\text { Calc. } \\
\text { (C) }\end{array}$ & $\begin{array}{c}\text { Exp. } \\
\text { (E) }\end{array}$ & $\mathrm{C} / \mathrm{E}$ \\
\hline $6.775 \mathrm{E}+01$ & 0.0515 & 0.0322 & 1.60 & $3.081 \mathrm{E}+02$ & 0.6565 & 0.4349 & 1.51 & $9.165 \mathrm{E}+02$ & 0.2887 & 0.3519 & 0.82 \\
\hline $7.285 \mathrm{E}+01$ & 0.0609 & 0.0597 & 1.02 & $3.317 \mathrm{E}+02$ & 0.5969 & 0.3983 & 1.50 & $9.870 \mathrm{E}+02$ & 0.2826 & 0.3413 & 0.83 \\
\hline $7.850 \mathrm{E}+01$ & 0.0721 & 0.0593 & 1.22 & $3.568 \mathrm{E}+02$ & 0.5453 & 0.4223 & 1.29 & $1.063 \mathrm{E}+03$ & 0.2841 & 0.2845 & 1.00 \\
\hline $8.415 \mathrm{E}+01$ & 0.0847 & 0.0646 & 1.31 & $3.833 \mathrm{E}+02$ & 0.4761 & 0.3139 & 1.52 & $1.142 \mathrm{E}+03$ & 0.2832 & 0.3055 & 0.93 \\
\hline $9.035 \mathrm{E}+01$ & 0.0993 & 0.0622 & 1.60 & $4.110 \mathrm{E}+02$ & 0.3888 & 0.2181 & 1.78 & $1.226 \mathrm{E}+03$ & 0.2706 & 0.2992 & 0.90 \\
\hline $9.710 \mathrm{E}+01$ & 0.1071 & 0.0633 & 1.69 & $4.423 \mathrm{E}+02$ & 0.3123 & 0.2576 & 1.21 & $1.320 \mathrm{E}+03$ & 0.2429 & 0.2276 & 1.07 \\
\hline $1.044 \mathrm{E}+02$ & 0.1171 & 0.0692 & 1.69 & $4.762 \mathrm{E}+02$ & 0.3417 & 0.3125 & 1.09 & $1.419 \mathrm{E}+03$ & 0.2071 & 0.1968 & 1.05 \\
\hline $1.123 \mathrm{E}+02$ & 0.1283 & 0.0974 & 1.32 & $5.130 \mathrm{E}+02$ & 0.3739 & 0.3392 & 1.10 & $1.524 \mathrm{E}+03$ & 0.1706 & 0.1713 & 1.00 \\
\hline $1.208 \mathrm{E}+02$ & 0.1407 & 0.1298 & 1.08 & $5.514 \mathrm{E}+02$ & 0.4094 & 0.3800 & 1.08 & $1.638 \mathrm{E}+03$ & 0.1654 & 0.1656 & 1.00 \\
\hline $1.299 \mathrm{E}+02$ & 0.1543 & 0.1819 & 0.85 & $5.927 \mathrm{E}+02$ & 0.4918 & 0.5574 & 0.88 & $1.761 \mathrm{E}+03$ & 0.1572 & 0.1635 & 0.96 \\
\hline $1.395 \mathrm{E}+02$ & 0.1691 & 0.1849 & 0.91 & $6.369 \mathrm{E}+02$ & 0.5904 & 0.6447 & 0.92 & $1.896 \mathrm{E}+03$ & 0.1414 & 0.1365 & 1.04 \\
\hline $1.496 \mathrm{E}+02$ & 0.1857 & 0.1528 & 1.22 & $6.840 \mathrm{E}+02$ & 0.6522 & 0.5391 & 1.21 & $2.039 \mathrm{E}+03$ & 0.1198 & 0.1051 & 1.14 \\
\hline $1.609 \mathrm{E}+02$ & 0.1831 & 0.1674 & 1.09 & $7.356 \mathrm{E}+02$ & 0.4417 & 0.3519 & 1.26 & $2.191 \mathrm{E}+03$ & 0.1136 & 0.0991 & 1.15 \\
\hline $1.734 \mathrm{E}+02$ & 0.1903 & 0.2096 & 0.91 & $7.917 \mathrm{E}+02$ & 0.2272 & 0.3195 & 0.71 & $2.355 \mathrm{E}+03$ & 0.1127 & 0.0883 & 1.28 \\
\hline $1.863 \mathrm{E}+02$ & 0.2401 & 0.2256 & 1.06 & $8.521 \mathrm{E}+02$ & 0.2521 & 0.3068 & 0.82 & $2.533 \mathrm{E}+03$ & 0.0802 & 0.0719 & 1.12 \\
\hline $2.004 \mathrm{E}+02$ & 0.2574 & 0.1645 & 1.56 & $9.155 \mathrm{E}+02$ & 0.2787 & 0.3505 & 0.80 & $2.726 \mathrm{E}+03$ & 0.0700 & 0.0639 & 1.10 \\
\hline $2.157 \mathrm{E}+02$ & 0.2699 & 0.2067 & 1.31 & $9.850 \mathrm{E}+02$ & 0.2829 & 0.3181 & 0.89 & $2.931 \mathrm{E}+03$ & 0.0622 & 0.0559 & 1.11 \\
\hline $2.315 \mathrm{E}+02$ & 0.2951 & 0.2067 & 1.43 & $1.060 \mathrm{E}+03$ & 0.2846 & 0.3068 & 0.93 & $3.150 \mathrm{E}+03$ & 0.0526 & 0.0459 & 1.14 \\
\hline $2.484 E+02$ & 0.3274 & 0.2475 & 1.32 & $1.139 \mathrm{E}+03$ & 0.2840 & 0.3209 & 0.88 & $3.387 \mathrm{E}+03$ & 0.0420 & 0.0426 & 0.99 \\
\hline $2.677 \mathrm{E}+02$ & 0.3382 & 0.3130 & 1.08 & $1.225 \mathrm{E}+03$ & 0.2712 & 0.2463 & 1.10 & $3.641 \mathrm{E}+03$ & 0.0395 & 0.0316 & 1.25 \\
\hline $2.880 \mathrm{E}+02$ & 0.3652 & 0.3465 & 1.05 & $1.316 \mathrm{E}+03$ & 0.2454 & 0.2322 & 1.06 & $3.916 \mathrm{E}+03$ & 0.0360 & 0.0261 & 1.38 \\
\hline $3.095 \mathrm{E}+02$ & 0.1961 & 0.4280 & 0.46 & $1.415 \mathrm{E}+03$ & 0.2098 & 0.2111 & 0.99 & $4.212 \mathrm{E}+03$ & 0.0311 & 0.0230 & 1.35 \\
\hline $3.326 \mathrm{E}+02$ & 0.2111 & 0.3727 & 0.57 & $1.523 \mathrm{E}+03$ & 0.1710 & 0.1858 & 0.92 & $4.527 \mathrm{E}+03$ & 0.0275 & 0.0180 & 1.53 \\
\hline $3.575 \mathrm{E}+02$ & 0.2280 & 0.3814 & 0.60 & $1.638 \mathrm{E}+03$ & 0.1657 & 0.1731 & 0.96 & $4.869 \mathrm{E}+03$ & 0.0244 & 0.0139 & 1.76 \\
\hline $3.846 \mathrm{E}+02$ & 0.7138 & 0.2591 & 2.75 & $1.760 \mathrm{E}+03$ & 0.1574 & 0.1618 & 0.97 & $5.238 \mathrm{E}+03$ & 0.0195 & 0.0093 & 2.10 \\
\hline $4.139 \mathrm{E}+02$ & 0.7609 & 0.2067 & 3.68 & $1.893 \mathrm{E}+03$ & 0.1420 & 0.1141 & 1.24 & & & & \\
\hline $4.450 \mathrm{E}+02$ & 0.8218 & 0.2911 & 2.82 & $2.036 \mathrm{E}+03$ & 0.1204 & 0.0977 & 1.23 & & & & \\
\hline $4.783 E+02$ & 0.8889 & 0.3101 & 2.87 & $2.189 \mathrm{E}+03$ & 0.1133 & 0.1009 & 1.12 & & & & \\
\hline $5.144 \mathrm{E}+02$ & 0.3756 & 0.3130 & 1.20 & $2.354 \mathrm{E}+03$ & 0.1330 & 0.0735 & 1.81 & & & & \\
\hline $5.534 \mathrm{E}+02$ & 0.4024 & 0.4120 & 0.98 & & & & & & & & \\
\hline $5.952 \mathrm{E}+02$ & 0.4564 & 0.6435 & 0.71 & & & & & & & & \\
\hline $6.398 \mathrm{E}+02$ & 0.4928 & 0.5226 & 0.94 & & & & & & & & \\
\hline $6.878 \mathrm{E}+02$ & 0.5284 & 0.3930 & 1.34 & & & & & & & & \\
\hline $7.392 \mathrm{E}+02$ & 0.7802 & 0.3261 & 2.39 & & & & & & & & \\
\hline $7.951 \mathrm{E}+02$ & 0.8328 & 0.3261 & 2.55 & & & & & & & BUG96/35 & \\
\hline
\end{tabular}


Figure 5.2-13: Detector 1 Data Comparison for $35 \mathrm{~cm}$ Sphere

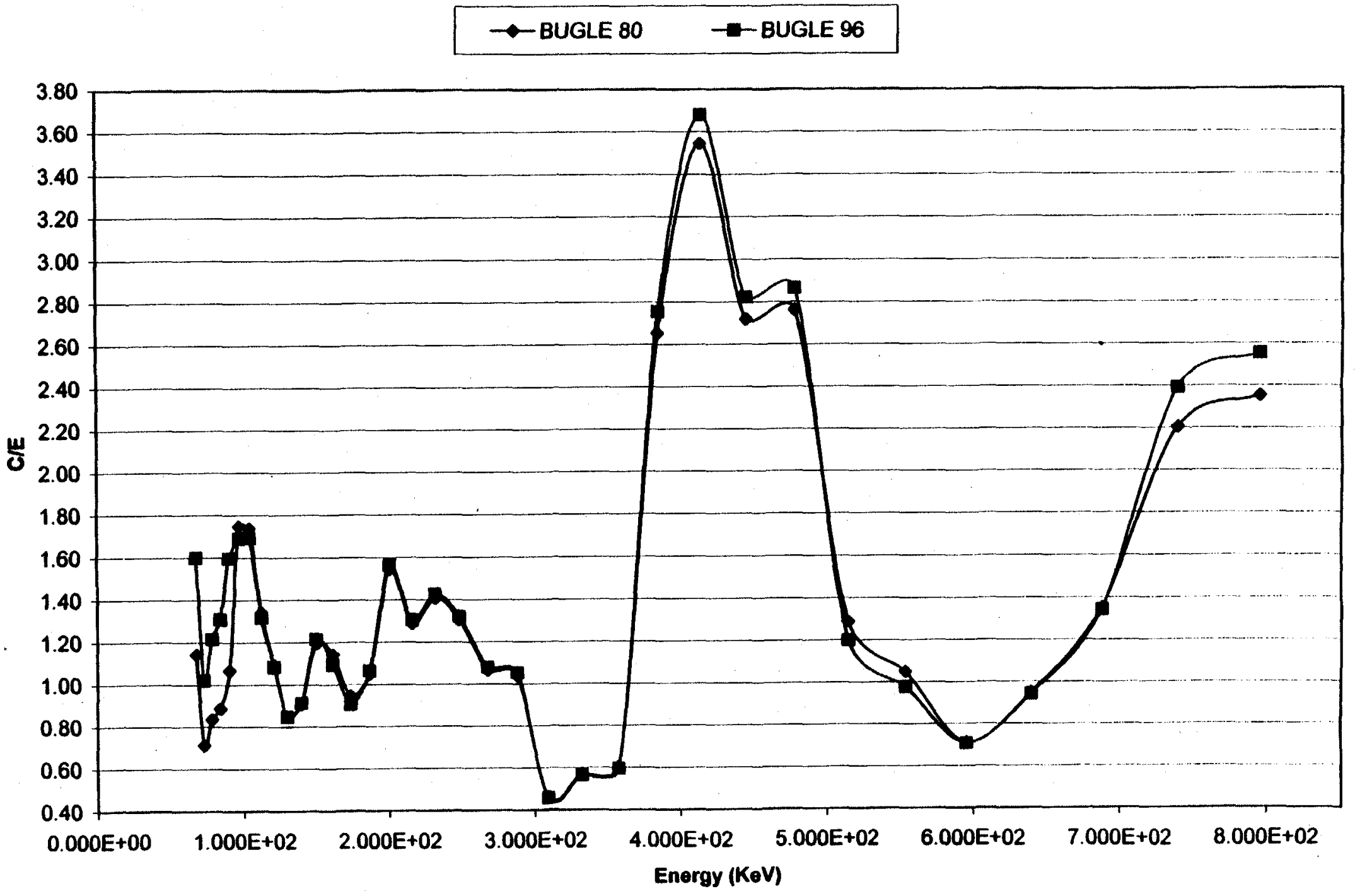


Figure 5.2-14: Detector 2 data comparison for $35 \mathrm{~cm}$ sphere.

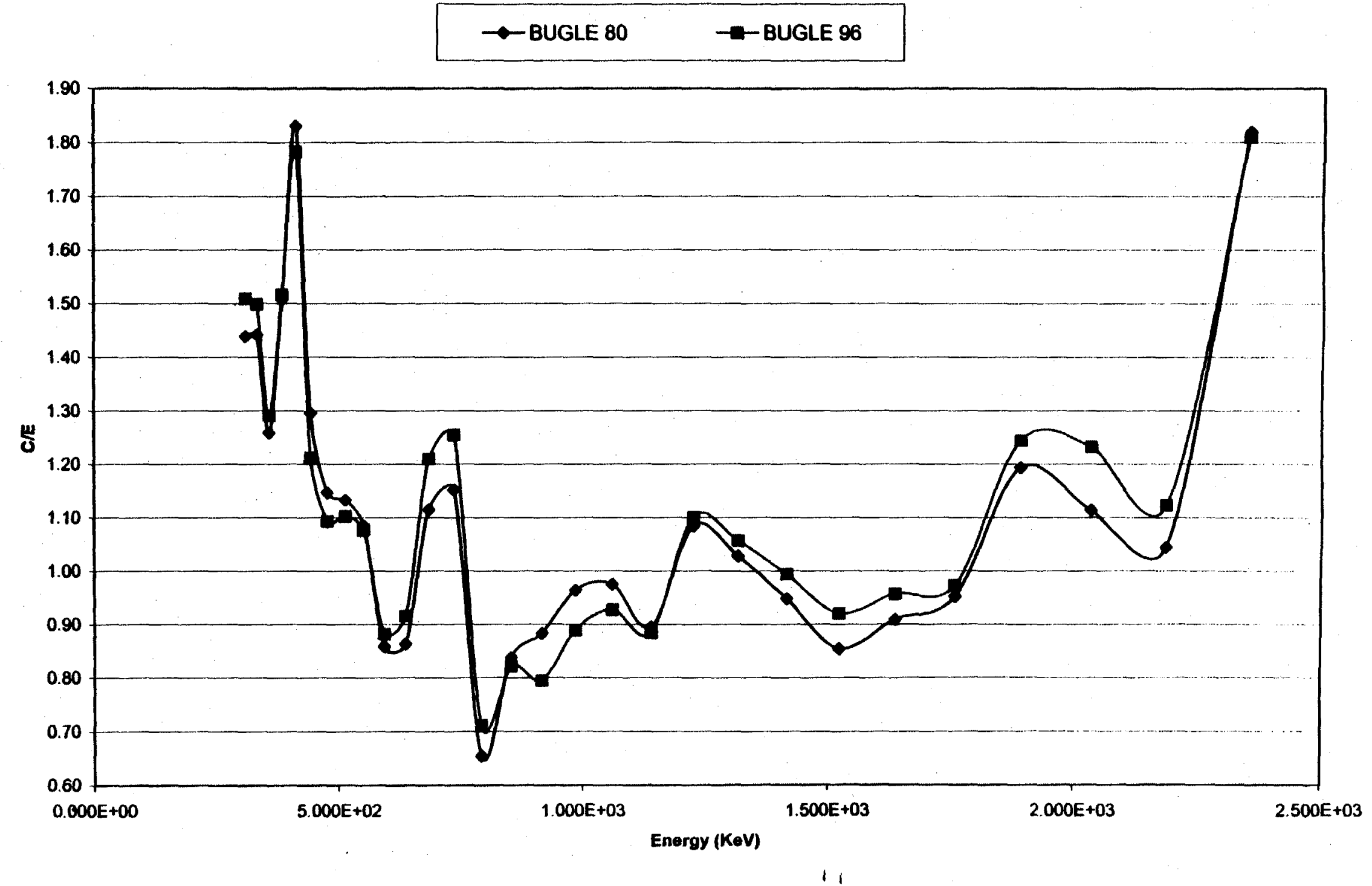


Figure 5.2-15: Detector 3 Data Comparison for $35 \mathrm{~cm}$ Sphere

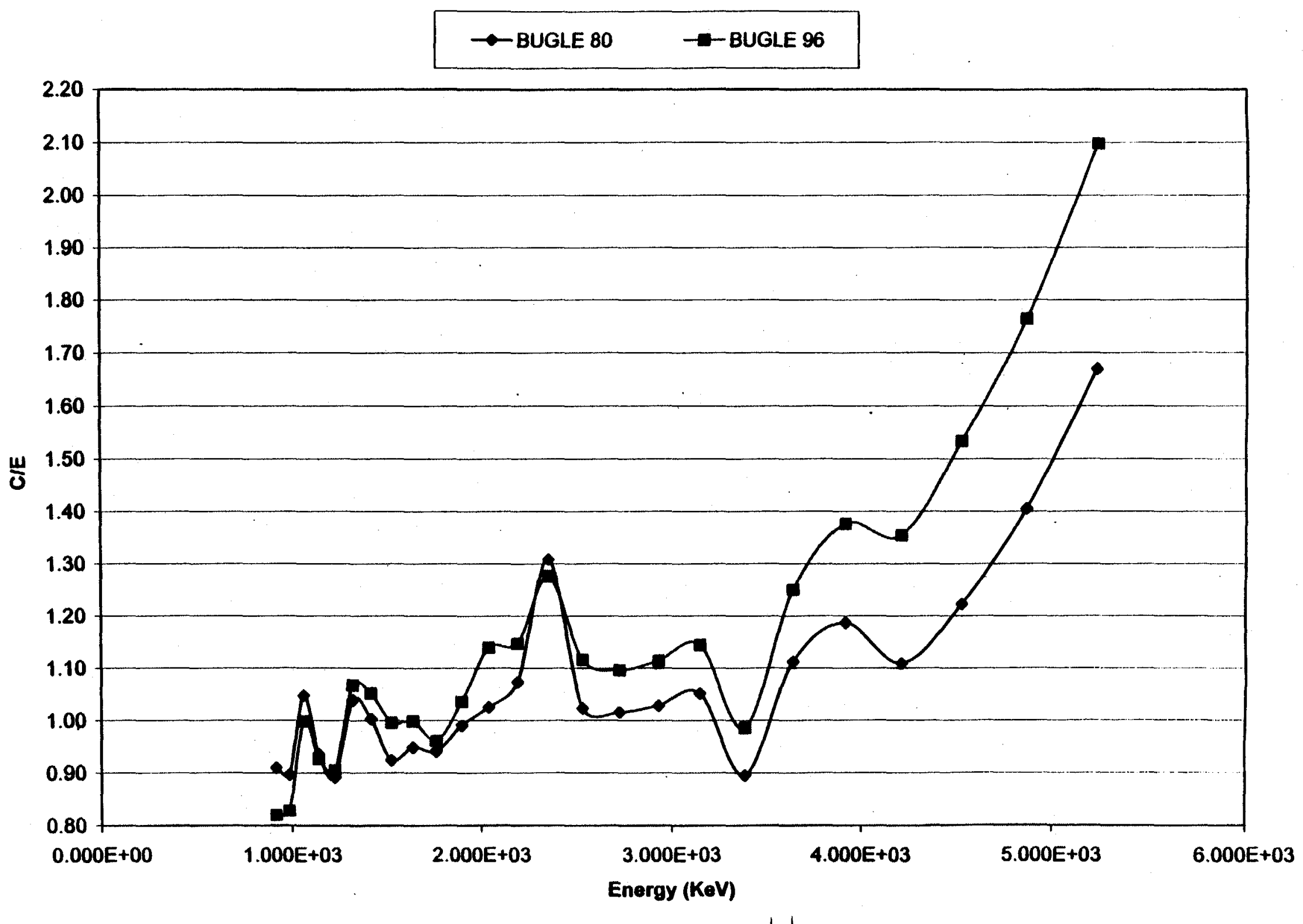


Table 5.2-11: Detector Measurements for $40 \mathrm{~cm}$ Diameter Iron Sphere using BUGLE 80 cross section library.

\begin{tabular}{|c|c|c|c|c|c|c|c|c|c|c|c|}
\hline \multicolumn{4}{|c|}{ Detector 1} & \multicolumn{4}{|c|}{ Detector 2} & \multicolumn{4}{|c|}{ Detector 3} \\
\hline $\begin{array}{c}\text { Mid Energy } \\
(\mathrm{keV})\end{array}$ & $\begin{array}{c}\text { Calc. } \\
(\mathrm{C})\end{array}$ & $\begin{array}{c}\text { Exp. } \\
\text { (E) }\end{array}$ & $\mathrm{C} / \mathrm{E}$ & $\begin{array}{c}\text { Mid Energy } \\
(\mathrm{keV})\end{array}$ & $\begin{array}{c}\text { Calc. } \\
\text { (C) }\end{array}$ & $\begin{array}{l}\text { Exp. } \\
\text { (E) }\end{array}$ & $\mathrm{C} / \mathrm{E}$ & $\begin{array}{c}\text { Mid Energy } \\
(\mathrm{keV})\end{array}$ & $\begin{array}{l}\text { Calc. } \\
\text { (C) }\end{array}$ & $\begin{array}{l}\text { Exp. } \\
\text { (E) }\end{array}$ & $\mathrm{C} / \mathrm{E}$ \\
\hline $6.775 \mathrm{E}+01$ & 0.0698 & 0.0479 & 1.46 & $3.081 \mathrm{E}+02$ & 0.6879 & 0.4417 & 1.56 & $9.165 \mathrm{E}+02$ & 0.2807 & 0.3247 & 0.86 \\
\hline $7.285 \mathrm{E}+01$ & 0.0802 & 0.0688 & 1.17 & $3.317 \mathrm{E}+02$ & 0.6267 & 0.3784 & 1.66 & $9.870 \mathrm{E}+02$ & 0.2651 & 0.3205 & 0.83 \\
\hline $7.850 \mathrm{E}+01$ & 0.0923 & 0.0803 & 1.15 & $3.568 \mathrm{E}+02$ & 0.5742 & 0.4149 & 1.38 & $1.063 \mathrm{E}+03$ & 0.2546 & 0.2551 & 1.00 \\
\hline $8.415 E+01$ & 0.1059 & 0.0905 & 1.17 & $3.833 \mathrm{E}+02$ & 0.5036 & 0.3108 & 1.62 & $1.142 \mathrm{E}+03$ & 0.2396 & 0.2678 & 0.89 \\
\hline $9.035 \mathrm{E}+01$ & 0.1216 & 0.0976 & 1.25 & $4.110 \mathrm{E}+02$ & 0.4144 & 0.2278 & 1.82 & $1.226 \mathrm{E}+03$ & 0.2199 & 0.2572 & 0.86 \\
\hline $9.710 \mathrm{E}+01$ & 0.1293 & 0.0687 & 1.88 & $4.423 E+02$ & 0.3342 & 0.2799 & 1.19 & $1.320 \mathrm{E}+03$ & 0.1922 & 0.1927 & 1.00 \\
\hline $1.044 \mathrm{E}+02$ & 0.1393 & 0.0857 & 1.63 & $4.762 \mathrm{E}+02$ & 0.3565 & 0.3249 & 1.10 & $1.419 \mathrm{E}+03$ & 0.1567 & 0.1607 & 0.98 \\
\hline $1.123 \mathrm{E}+02$ & 0.1501 & 0.1181 & 1.27 & $5.130 \mathrm{E}+02$ & 0.3797 & 0.3277 & 1.16 & $1.524 \mathrm{E}+03$ & 0.1215 & 0.1415 & 0.86 \\
\hline $1.208 \mathrm{E}+02$ & 0.1618 & 0.1415 & 1.14 & $5.514 \mathrm{E}+02$ & 0.4043 & 0.4065 & 0.99 & $1.638 \mathrm{E}+03$ & 0.1198 & 0.1282 & 0.93 \\
\hline $1.299 \mathrm{E}+02$ & 0.1742 & 0.1792 & 0.97 & $5.927 \mathrm{E}+02$ & 0.4673 & 0.5851 & 0.80 & $1.761 \mathrm{E}+03$ & 0.1164 & 0.1282 & 0.91 \\
\hline $1.395 \mathrm{E}+02$ & 0.1875 & 0.2433 & 0.77 & $6.369 \mathrm{E}+02$ & 0.5415 & 0.6259 & 0.87 & $1.896 \mathrm{E}+03$ & 0.1010 & 0.1031 & 0.98 \\
\hline $1.496 \mathrm{E}+02$ & 0.2020 & 0.2006 & 1.01 & $6.840 \mathrm{E}+02$ & 0.5811 & 0.4009 & 1.45 & $2.039 \mathrm{E}+03$ & 0.0786 & 0.0810 & 0.97 \\
\hline $1.609 \mathrm{E}+02$ & 0.2249 & 0.1629 & 1.38 & $7.356 \mathrm{E}+02$ & 0.3808 & 0.3418 & 1.11 & $2.191 \mathrm{E}+03$ & 0.0771 & 0.0726 & 1.06 \\
\hline $1.734 \mathrm{E}+02$ & 0.2338 & 0.2342 & 1.00 & $7.917 \mathrm{E}+02$ & 0.1794 & 0.3094 & 0.58 & $2.355 \mathrm{E}+03$ & 0.0844 & 0.0614 & 1.38 \\
\hline $1.863 \mathrm{E}+02$ & 0.2624 & 0.2484 & 1.06 & $8.521 \mathrm{E}+02$ & 0.2235 & 0.2560 & 0.87 & $2.533 \mathrm{E}+03$ & 0.0511 & 0.0527 & 0.97 \\
\hline $2.004 \mathrm{E}+02$ & 0.2812 & 0.1924 & 1.46 & $9.155 \mathrm{E}+02$ & 0.2720 & 0.3263 & 0.83 & $2.726 \mathrm{E}+03$ & 0.0449 & 0.0441 & 1.02 \\
\hline $2.157 \mathrm{E}+02$ & 0.2949 & 0.1975 & 1.49 & $9.850 \mathrm{E}+02$ & 0.2664 & 0.2968 & 0.90 & $2.931 E+03$ & 0.0394 & 0.0390 & 1.01 \\
\hline $2.315 \mathrm{E}+02$ & 0.3224 & 0.2546 & 1.27 & $1.060 \mathrm{E}+03$ & 0.2561 & 0.2349 & 1.09 & $3.150 \mathrm{E}+03$ & 0.0329 & 0.0323 & 1.02 \\
\hline $2.484 \mathrm{E}+02$ & 0.3577 & 0.2658 & 1.35 & $1.139 E+03$ & 0.2412 & 0.2757 & 0.87 & $3.387 \mathrm{E}+03$ & 0.0257 & 0.0278 & 0.92 \\
\hline $2.677 \mathrm{E}+02$ & 0.3695 & 0.3401 & 1.09 & $1.225 \mathrm{E}+03$ & 0.2204 & 0.2067 & 1.07 & $3.641 \mathrm{E}+03$ & 0.0236 & 0.0232 & 1.02 \\
\hline $2.880 \mathrm{E}+02$ & 0.3990 & 0.3788 & 1.05 & $1.316 \mathrm{E}+03$ & 0.1942 & 0.1730 & 1.12 & $3.916 \mathrm{E}+03$ & 0.0207 & 0.0170 & 1.22 \\
\hline $3.095 \mathrm{E}+02$ & 0.2029 & 0.4715 & 0.43 & $1.415 \mathrm{E}+03$ & 0.1588 & 0.1547 & 1.03 & $4.212 \mathrm{E}+03$ & 0.0169 & 0.0153 & 1.10 \\
\hline $3.326 \mathrm{E}+02$ & 0.2184 & 0.4195 & 0.52 & $1.523 \mathrm{E}+03$ & 0.1212 & 0.1399 & 0.87 & $4.527 \mathrm{E}+03$ & 0.0145 & 0.0128 & 1.13 \\
\hline $3.575 \mathrm{E}+02$ & 0.2360 & 0.4318 & 0.55 & $1.638 \mathrm{E}+03$ & 0.1199 & 0.1448 & 0.83 & $4.869 \mathrm{E}+03$ & 0.0129 & 0.0082 & 1.57 \\
\hline $3.846 \mathrm{E}+02$ & 0.7495 & 0.3330 & 2.25 & $1.760 \mathrm{E}+03$ & 0.1170 & 0.1129 & 1.04 & $5.238 \mathrm{E}+03$ & 0.0103 & 0.0068 & 1.52 \\
\hline $4.139 \mathrm{E}+02$ & 0.7990 & 0.1985 & 4.03 & $1.893 \mathrm{E}+03$ & 0.1022 & 0.0979 & 1.04 & & & & \\
\hline $4.450 \mathrm{E}+02$ & 0.8629 & 0.2454 & 3.52 & $2.036 \mathrm{E}+03$ & 0.1088 & 0.0713 & 1.53 & & & & \\
\hline $4.783 E+02$ & 0.9335 & 0.3421 & 2.73 & $2.189 \mathrm{E}+03$ & 0.0776 & 0.0640 & 1.21 & & & & \\
\hline $5.144 \mathrm{E}+02$ & 0.4045 & 0.3279 & 1.23 & $2.354 \mathrm{E}+03$ & 0.0939 & 0.0521 & 1.80 & & & & \\
\hline $5.534 \mathrm{E}+02$ & 0.4334 & 0.3992 & 1.09 & & & & & & & & \\
\hline $5.952 \mathrm{E}+02$ & 0.4502 & 0.5906 & 0.76 & & & & & & & & \\
\hline $6.398 \mathrm{E}+02$ & 0.4861 & 0.6110 & 0.80 & & & & & & & & \\
\hline $6.878 \mathrm{E}+02$ & 0.5212 & 0.4440 & 1.17 & & & & & & & & \\
\hline $7.392 \mathrm{E}+02$ & 0.6970 & 0.2820 & 2.47 & & & & & & & & \\
\hline $7.951 \mathrm{E}+02$ & 0.7440 & 0.3218 & 2.31 & & & & & & & BUG $80 / 40$ & \\
\hline
\end{tabular}


Table 5.2-12: Detector Measurements for $40 \mathrm{~cm}$ Diameter Iron Sphere using BUGLE 96 cross section library.

\begin{tabular}{|c|c|c|c|c|c|c|c|c|c|c|c|}
\hline \multicolumn{4}{|c|}{ Detector 1} & \multicolumn{4}{|c|}{ Detector 2} & \multicolumn{4}{|c|}{ Detector 3} \\
\hline $\begin{array}{l}\text { Mid Energy } \\
\text { (keV) }\end{array}$ & $\begin{array}{l}\text { Calc. } \\
\text { (C) }\end{array}$ & $\begin{array}{l}\text { Exp. } \\
\text { (E) }\end{array}$ & $\mathrm{C} / \mathrm{E}$ & $\begin{array}{l}\text { Mid Energy } \\
\text { (keV) }\end{array}$ & $\begin{array}{l}\text { Calc. } \\
\text { (C) }\end{array}$ & $\begin{array}{l}\text { Exp. } \\
\text { (E) }\end{array}$ & $\mathrm{C} / \mathrm{E}$ & $\begin{array}{l}\text { Mid Energy } \\
\text { (keV) }\end{array}$ & $\begin{array}{l}\text { Calc. } \\
\text { (C) }\end{array}$ & $\begin{array}{l}\text { Exp. } \\
\text { (E) }\end{array}$ & $\mathrm{C} / \mathrm{E}$ \\
\hline $6.775 \mathrm{E}+01$ & 0.0629 & 0.0479 & 1.31 & $3.081 E+02$ & 0.7309 & 0.4417 & 1.65 & $9.165 \mathrm{E}+02$ & 0.2515 & 0.3247 & 0.77 \\
\hline $7.285 \mathrm{E}+01$ & 0.0744 & 0.0688 & 1.08 & $3.317 \mathrm{E}+02$ & 0.6592 & 0.3784 & 1.74 & $9.870 \mathrm{E}+02$ & 0.2436 & 0.3205 & 0.76 \\
\hline $7.850 \mathrm{E}+01$ & 0.0880 & 0.0803 & 1.10 & $3.568 \mathrm{E}+02$ & 0.5952 & 0.4149 & 1.43 & $1.063 \mathrm{E}+03$ & 0.2416 & 0.2551 & 0.95 \\
\hline $8.415 \mathrm{E}+01$ & 0.1034 & 0.0905 & 1.14 & $3.833 E+02$ & 0.5103 & 0.3108 & 1.64 & $1.142 \mathrm{E}+03$ & 0.2369 & 0.2678 & 0.88 \\
\hline $9.035 \mathrm{E}+01$ & 0.1212 & 0.0976 & 1.24 & $4.110 \mathrm{E}+02$ & 0.4038 & 0.2278 & 1.77 & $1.226 \mathrm{E}+03$ & 0.2238 & 0.2572 & 0.87 \\
\hline $9.710 \mathrm{E}+01$ & 0.1294 & 0.0687 & 1.88 & $4.423 \mathrm{E}+02$ & 0.3100 & 0.2799 & 1.11 & $1.320 \mathrm{E}+03$ & 0.1986 & 0.1927 & 1.03 \\
\hline $1.044 E+02$ & 0.1402 & 0.0857 & 1.64 & $4.762 \mathrm{E}+02$ & 0.3391 & 0.3249 & 1.04 & $1.419 \mathrm{E}+03$ & 0.1661 & 0.1607 & 1.03 \\
\hline $1.123 \mathrm{E}+02$ & 0.1521 & 0.1181 & 1.29 & $5.130 \mathrm{E}+02$ & 0.3710 & 0.3277 & 1.13 & $1.524 \mathrm{E}+03$ & 0.1328 & 0.1415 & 0.94 \\
\hline $1.208 \mathrm{E}+02$ & 0.1651 & 0.1415 & 1.17 & $5.514 \mathrm{E}+02$ & 0.4062 & 0.4065 & 1.00 & $1.638 \mathrm{E}+03$ & 0.1285 & 0.1282 & 1.00 \\
\hline $1.299 \mathrm{E}+02$ & 0.1792 & 0.1792 & 1.00 & $5.927 \mathrm{E}+02$ & 0.4838 & 0.5851 & 0.83 & $1.761 E+03$ & 0.1218 & 0.1282 & 0.95 \\
\hline $1.395 E+02$ & 0.1943 & 0.2433 & 0.80 & $6.369 \mathrm{E}+02$ & 0.5761 & 0.6259 & 0.92 & $1.896 \mathrm{E}+03$ & 0.1086 & 0.1031 & 1.05 \\
\hline $1.496 \mathrm{E}+02$ & 0.2111 & 0.2006 & 1.05 & $6.840 \mathrm{E}+02$ & 0.6313 & 0.4009 & 1.57 & $2.039 \mathrm{E}+03$ & 0.0905 & 0.0810 & 1.12 \\
\hline $1.609 \mathrm{E}+02$ & 0.2234 & 0.1629 & 1.37 & $7.356 \mathrm{E}+02$ & 0.4153 & 0.3418 & 1.22 & $2.191 \mathrm{E}+03$ & 0.0847 & 0.0726 & 1.17 \\
\hline $1.734 \mathrm{E}+02$ & 0.2323 & 0.2342 & 0.99 & $7.917 \mathrm{E}+02$ & 0.1945 & 0.3094 & 0.63 & $2.355 \mathrm{E}+03$ & 0.0829 & 0.0614 & 1.35 \\
\hline $1.863 \mathrm{E}+02$ & 0.2736 & 0.2484 & 1.10 & $8.521 \mathrm{E}+02$ & 0.2178 & 0.2560 & 0.85 & $2.533 \mathrm{E}+03$ & 0.0580 & 0.0527 & 1.10 \\
\hline $2.004 E+02$ & 0.2932 & 0.1924 & 1.52 & $9.155 \mathrm{E}+02$ & 0.2429 & 0.3263 & 0.74 & $2.726 \mathrm{E}+03$ & 0.0502 & 0.0441 & 1.14 \\
\hline $2.157 \mathrm{E}+02$ & 0.3075 & 0.1975 & 1.56 & $9.850 \mathrm{E}+02$ & 0.2439 & 0.2968 & 0.82 & $2.931 \mathrm{E}+03$ & 0.0441 & 0.0390 & 1.13 \\
\hline $2.315 \mathrm{E}+02$ & 0.3362 & 0.2546 & 1.32 & $1.060 \mathrm{E}+03$ & 0.2423 & 0.2349 & 1.03 & $3.150 \mathrm{E}+03$ & 0.0370 & 0.0323 & 1.15 \\
\hline $2.484 \mathrm{E}+02$ & 0.3730 & 0.2658 & 1.40 & $1.139 \mathrm{E}+03$ & 0.2381 & 0.2757 & 0.86 & $3.387 \mathrm{E}+03$ & 0.0292 & 0.0278 & 1.05 \\
\hline $2.677 E+02$ & 0.3854 & 0.3401 & 1.13 & $1.225 \mathrm{E}+03$ & 0.2243 & 0.2067 & 1.09 & $3.641 \mathrm{E}+03$ & 0.0275 & 0.0232 & 1.18 \\
\hline $2.880 \mathrm{E}+02$ & 0.4161 & 0.3788 & 1.10 & $1.316 \mathrm{E}+03$ & 0.2006 & 0.1730 & 1.16 & $3.916 \mathrm{E}+03$ & 0.0250 & 0.0170 & 1.47 \\
\hline $3.095 \mathrm{E}+02$ & 0.2058 & 0.4715 & 0.44 & $1.415 \mathrm{E}+03$ & 0.1682 & 0.1547 & 1.09 & $4.212 \mathrm{E}+03$ & 0.0216 & 0.0153 & 1.41 \\
\hline $3.326 \mathrm{E}+02$ & 0.2215 & 0.4195 & 0.53 & $1.523 \mathrm{E}+03$ & 0.1332 & 0.1399 & 0.95 & $4.527 \mathrm{E}+03$ & 0.0190 & 0.0128 & 1.48 \\
\hline $3.575 \mathrm{E}+02$ & 0.2393 & $\overline{0.4318}$ & 0.55 & $1.638 \mathrm{E}+03$ & 0.1286 & 0.1448 & 0.89 & $4.869 \mathrm{E}+03$ & 0.0168 & 0.0082 & 2.05 \\
\hline $3.846 \mathrm{E}+02$ & 0.7882 & 0.3330 & 2.37 & $1.760 \mathrm{E}+03$ & 0.1217 & 0.1129 & 1.08 & $5.238 \mathrm{E}+03$ & 0.0134 & 0.0068 & 1.97 \\
\hline $4.139 \mathrm{E}+02$ & 0.8402 & 0.1985 & 4.23 & $1.893 \mathrm{E}+03$ & 0.1087 & 0.0979 & 1.11 & & & & \\
\hline $4.450 \mathrm{E}+02$ & 0.9075 & 0.2454 & 3.70 & $2.036 \mathrm{E}+03$ & 0.0907 & 0.0713 & 1.27 & & & & \\
\hline $4.783 \mathrm{E}+02$ & 0.9816 & 0.3421 & 2.87 & $2.189 \mathrm{E}+03$ & 0.0850 & 0.0640 & 1.33 & & & & \\
\hline $5.144 \mathrm{E}+02$ & 0.3728 & 0.3279 & 1.14 & $2.354 \mathrm{E}+03$ & 0.0970 & 0.0521 & 1.86 & & & & \\
\hline $5.534 \mathrm{E}+02$ & 0.3994 & 0.3992 & 1.00 & & & & & & & & \\
\hline $5.952 \mathrm{E}+02$ & 0.4528 & 0.5906 & 0.77 & & & & & & & & \\
\hline $6.398 \mathrm{E}+02$ & 0.4889 & 0.6110 & 0.80 & & & & & & & & \\
\hline $6.878 \mathrm{E}+02$ & 0.5242 & 0.4440 & 1.18 & & & & & & & & \\
\hline $7.392 \mathrm{E}+02$ & 0.7567 & 0.2820 & 2.68 & & & & & & & & \\
\hline $7.951 \mathrm{E}+02$ & 0.8078 & 0.3218 & 2.51 & & & & & & & BUG96/40 & \\
\hline
\end{tabular}


Figure 5.2-16: Detector 1 Data Comparison for $40 \mathrm{~cm}$ Sphere

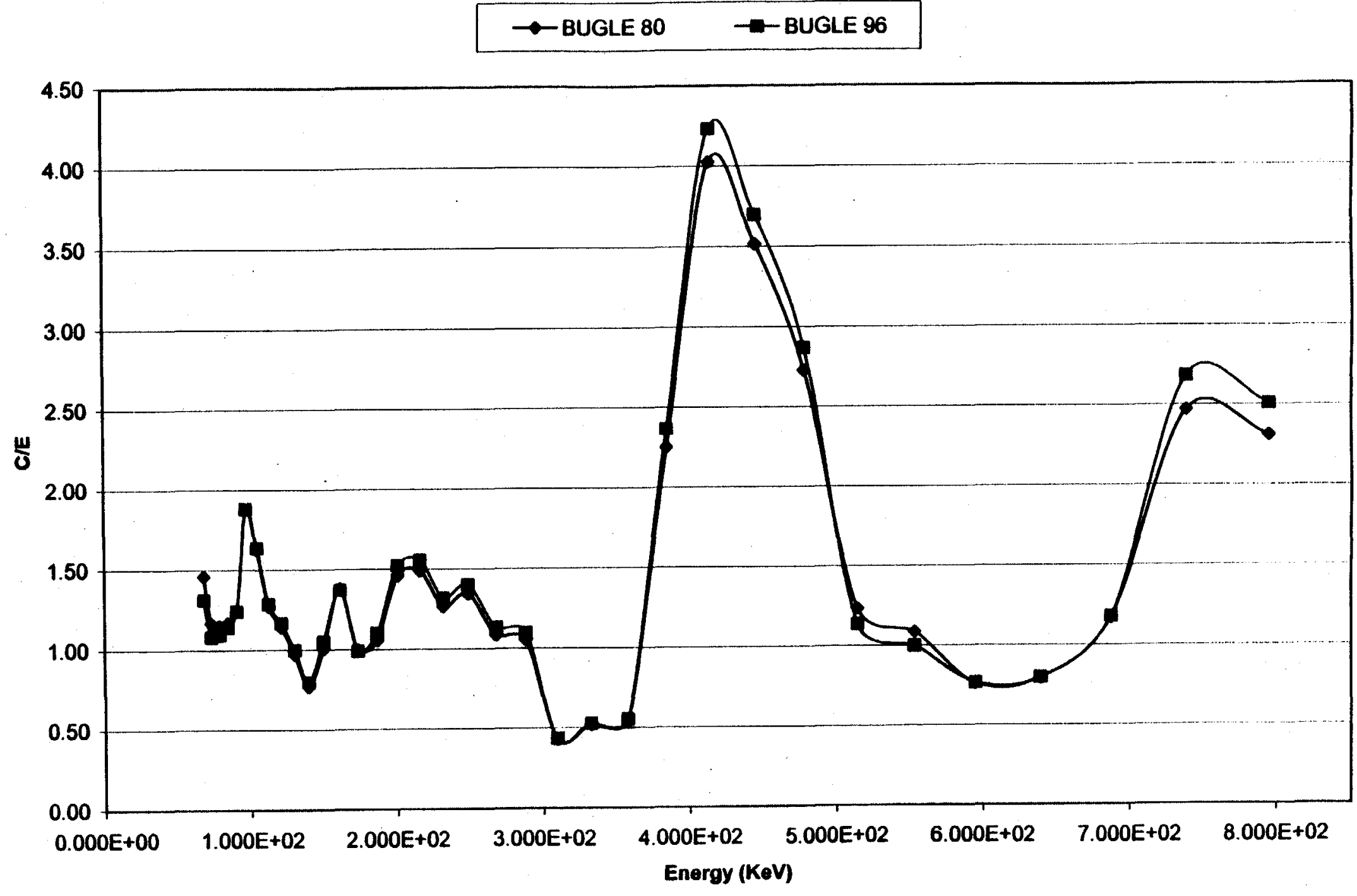


Figure 5.2-18: Detector 3 Data Comparison for $40 \mathrm{~cm}$ Sphere

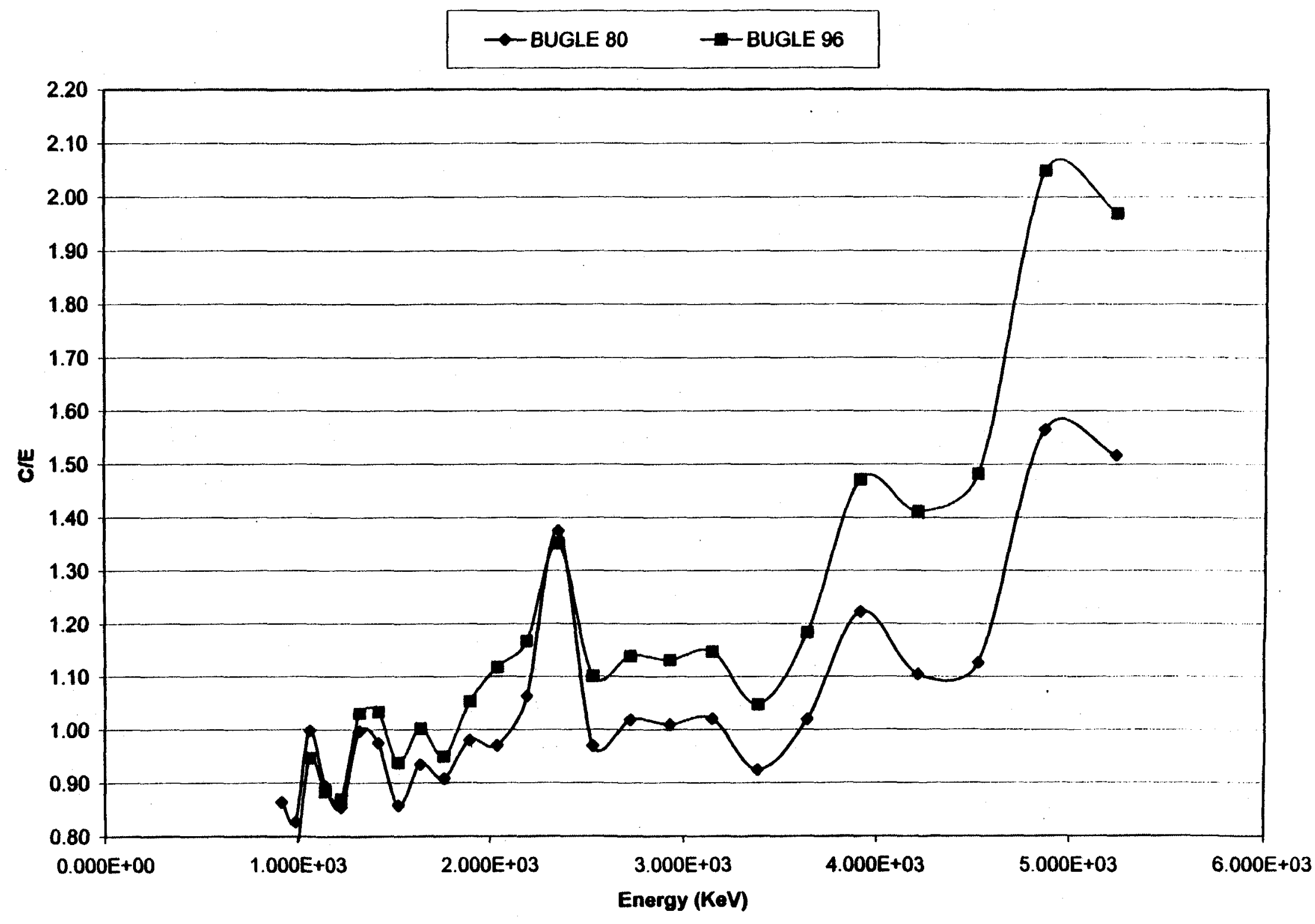

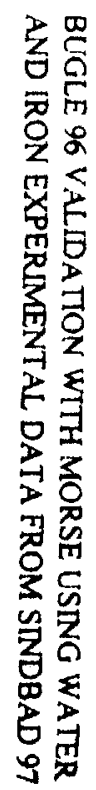




\subsection{Conclusion}

MORSE-SGC/S code was validated with the BUGLE-96 cross section set for the parameters described in Table 6.1. Two sets of benchmark measurement data were obtained from the SINBAD 97 database. The neutron spectrum measurement data in water was obtained from the Winfrith Water Benchmark Experiment and the leakage spectrum data in iron was obtained from the Karlsruhe Iron Sphere Benchmark Experiment. In both cases, the Cf-252 source was used and a standard Cf-252 source data (neutron spectrum) was taken from the University of Illinois Iron Sphere Benchmark evaluation in SINBAD 97 database, since it was given in 47 group BUGLE-96 format. All calculations with the BUGLE-96 cross section set were repeated with the BUGLE-80 set to determine the improvement (if any) in predicting the neutron spectrum due to the fact that BUGLE-96 was derived from the latest ENDF/B-VI evaluation.

The experimental spectra for neutron both in water and in iron were well predicted by MORSESGC/S with the BUGLE-96 cross section set. The differences between the calculated and measured values lie within a $20 \%$ range for most of the energy band in each case. The BUGLE= 96 generated spectra were close to those generated using the BUGLE-80 cross section set, and a distinct advantage of the BUGLE-96 set over the earlier cross section set was not determined from this limited validation study. This validation study should be continued with several other benchmark experiments available from the SINBAD 97 database to complete the validation of MORSE-SCG/S with the BUGLE-96 cross section set.

Table 6.1: MORSE-SGC/S Validation with BUGLE-96 cross sections

\begin{tabular}{|c|c|c|}
\hline Parameters & Water & Iron \\
\hline Material Thickness & Infinite & \begin{tabular}{c}
$7.5 \mathrm{~cm}$ to $20 \mathrm{~cm}$ \\
\hline Energy Range
\end{tabular} \\
\hline $\begin{array}{c}\text { Detector to Source } \\
\text { Distance (cm) }\end{array}$ & 0.9 to $8.8 \mathrm{MeV}$ & $\begin{array}{c}60 \mathrm{KeV} \text { to } 2 \mathrm{MeV}^{*}, \\
1.0-4 . \mathrm{MeV}^{* *}\end{array}$ \\
\hline
\end{tabular}

* Based on detectors at a distance of $102 \mathrm{~cm}$ from source.

** Based on detector at a distance of $117 \mathrm{~cm}$ from source. 


\subsection{References}

1. J. T. West et. al, MORSE-SCG/S for the SCALE system, NUREG/CR-0200, Rev.5.

2. DLC-185/ BUGLE-96, Coupled 47 Neutrons, 20 Gamma-Ray Group Cross Section Library Derived from ENDF/B-VI for LWR Shielding and Pressure Vessel Dosimetry Applications, Oak Ridge National Laboratory, March 1996.

3. SINBAD 97/DLC-191, Shielding Integral Benchmark Archive and Database, Oak Ridge National Laboratory, September 1997.

4. RSICC ,CCC-545, SCALE 4.3 Modular Code System for Performing Standardized Computer Analysis for Licensing Evaluation, Oak Ridge National Laboratory, March 1997.

5. WSMS-CTG-98-0009, "Software Configuration and Control Guidance for SCALE 4.3 (U)", G. Kessler, February, 1998.

6. M. J. Grimstone, The RADAK User's Manual, AEEW-M1455 (1976) 


\section{APPENDIX A: Input Listings for Winfrith Water Benchmark Experiment}

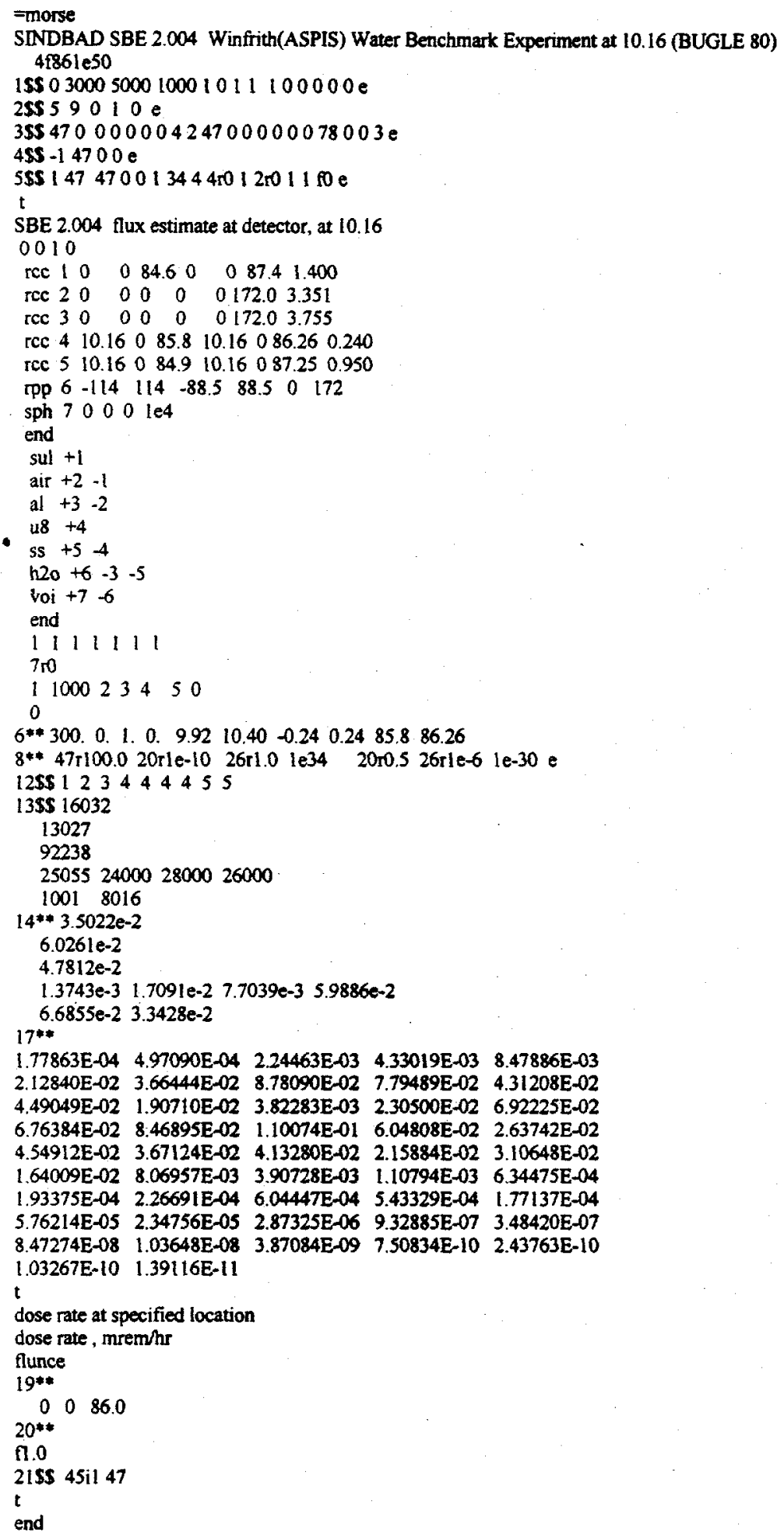




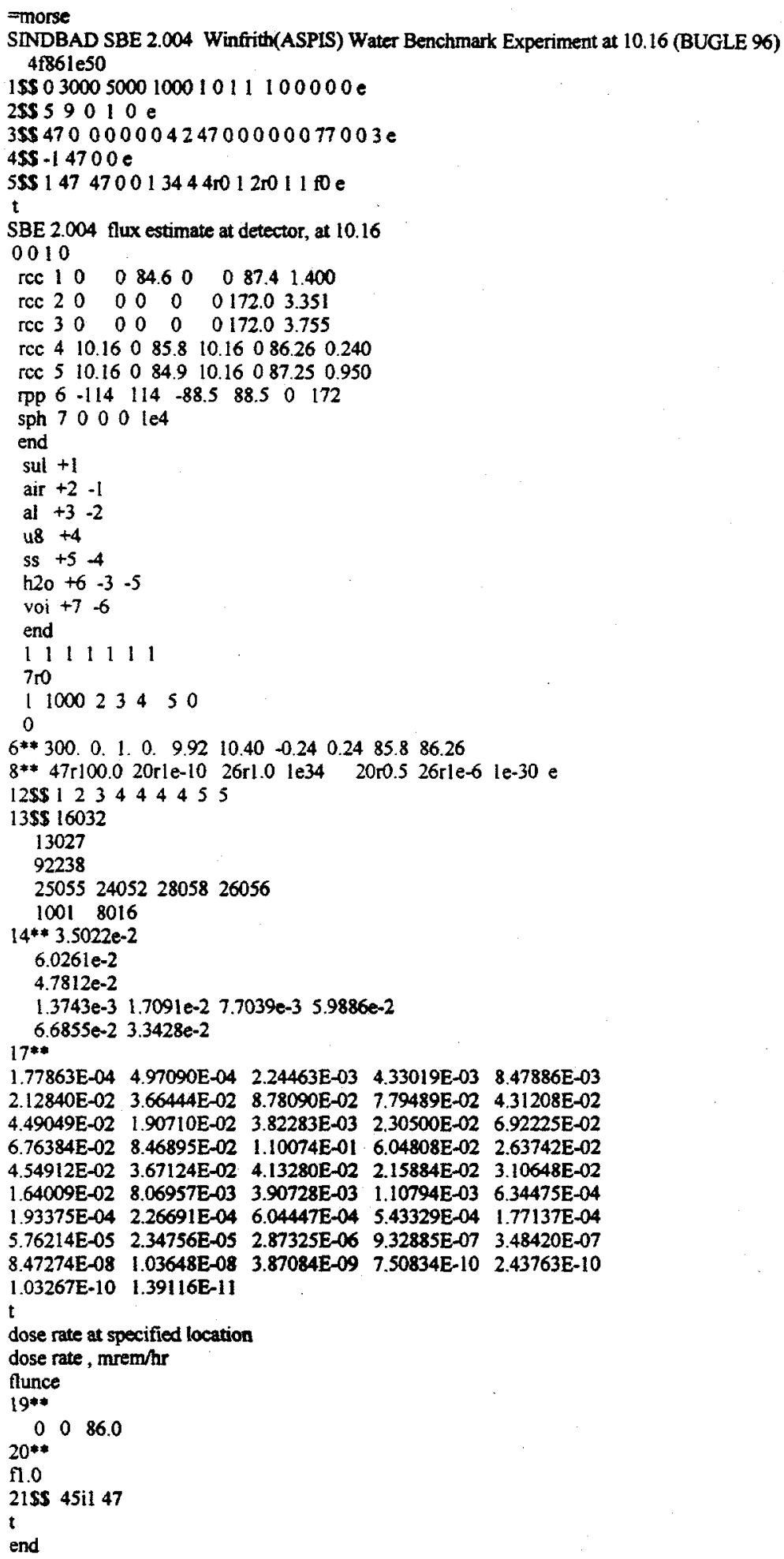


Rev.0

Page 84 of 89

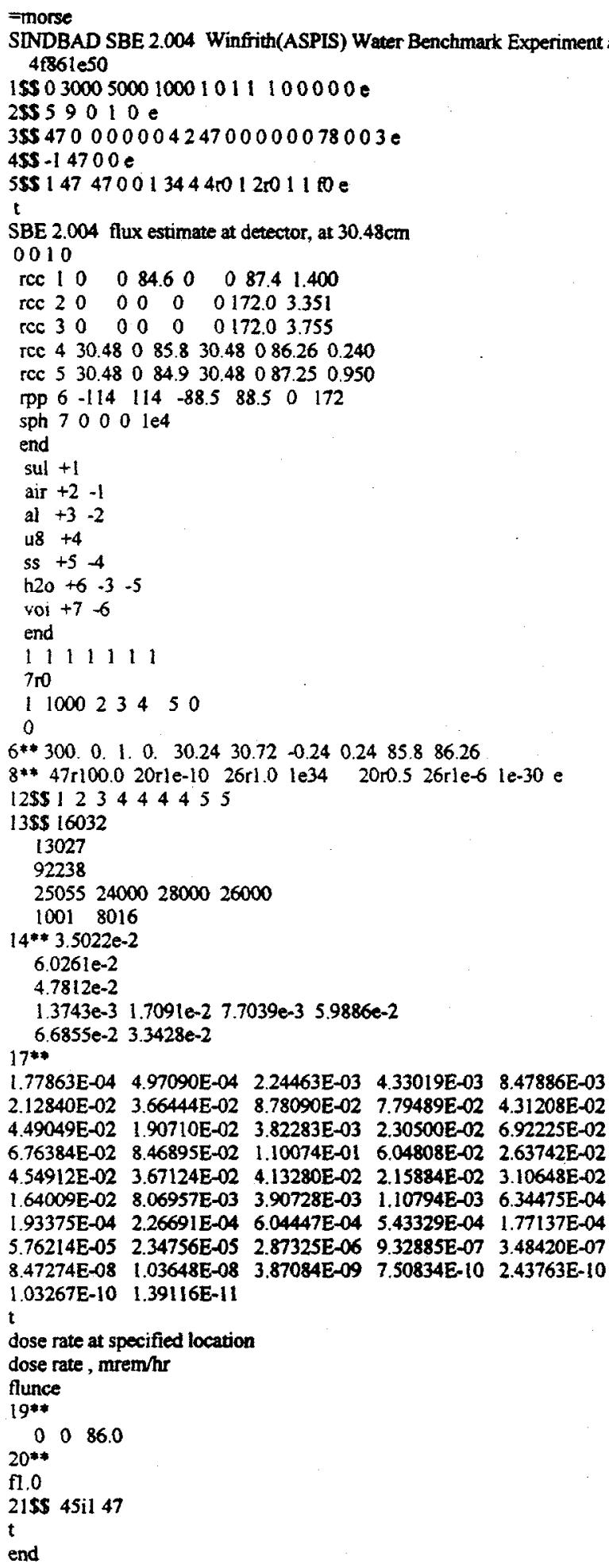




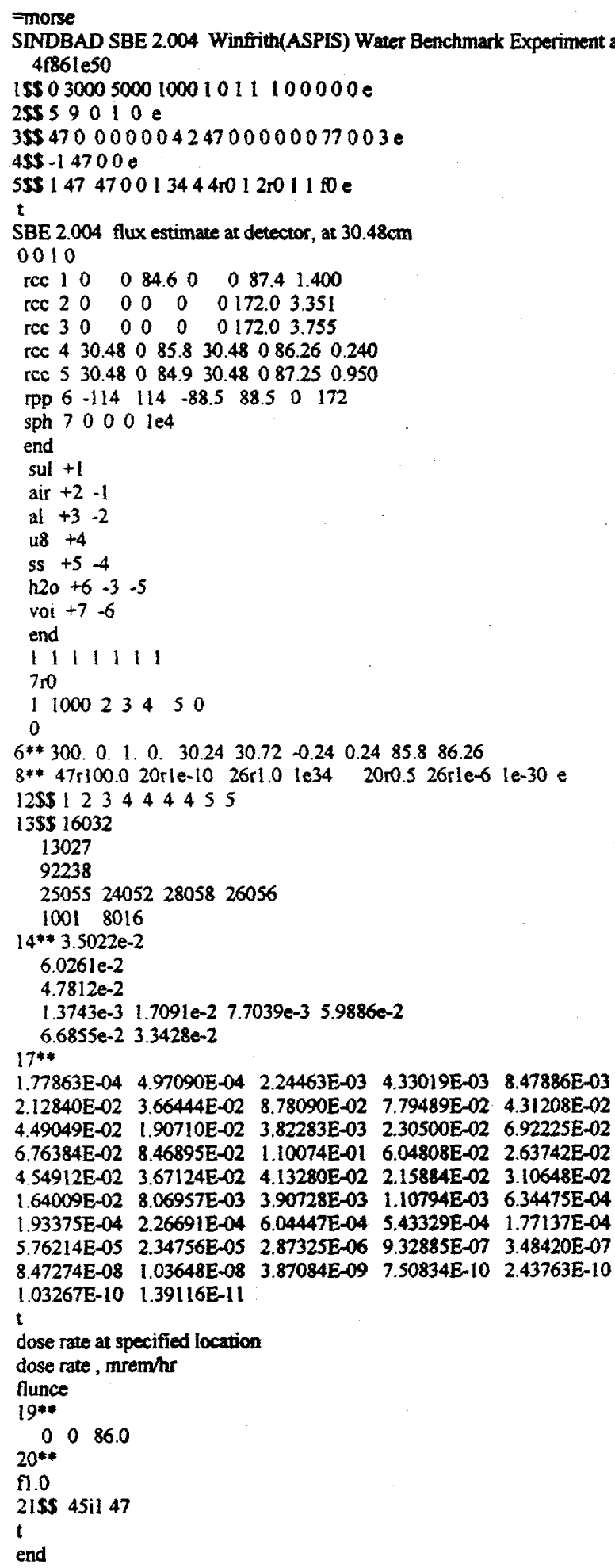




\section{APPENDIX B: Input Listings for Karlsruhe Iron Sphere Benchmark Experiment}

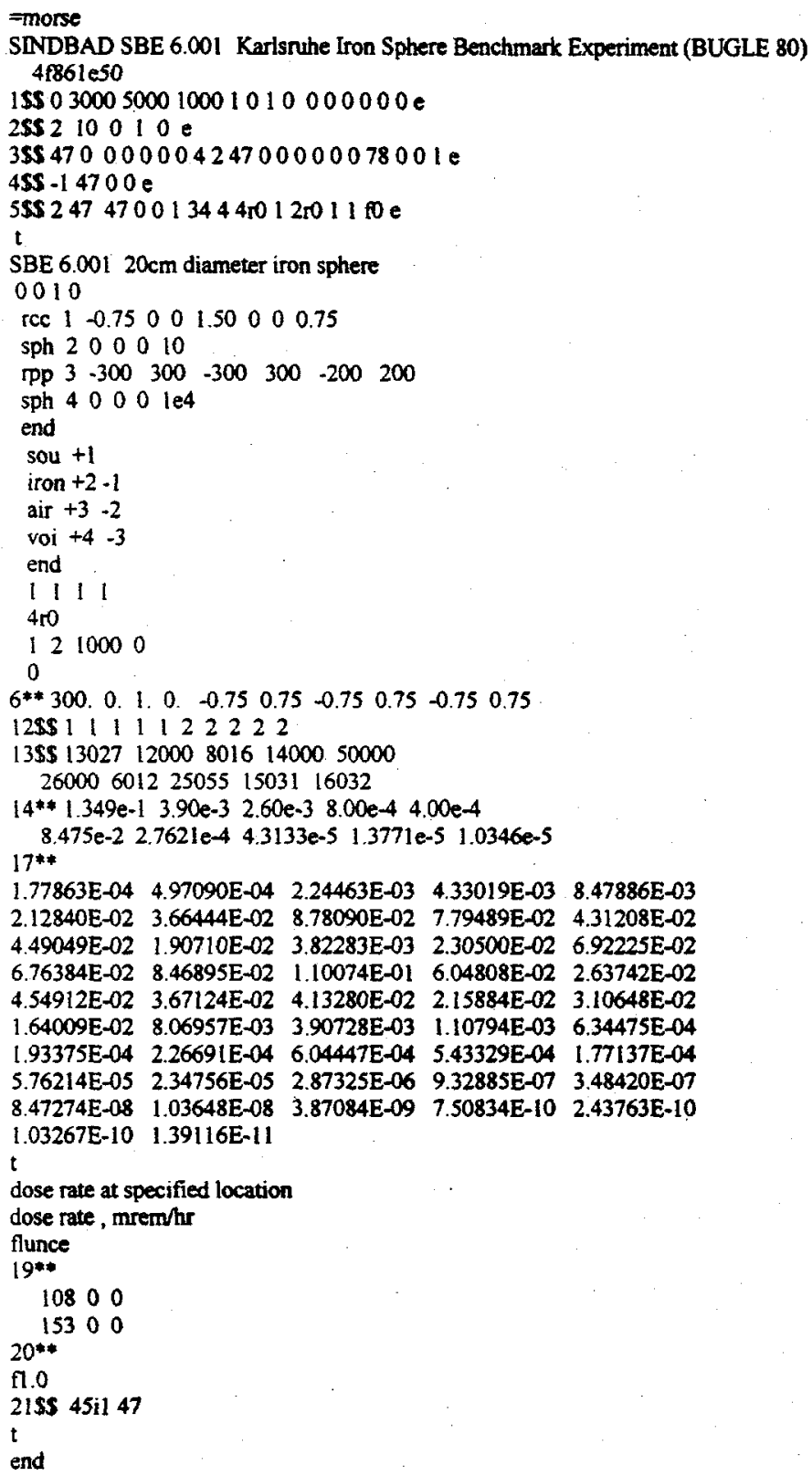


morse

SINDBAD SBE 6.001 Kartsnuhe Iron Sphere Benchmark Experiment (BUGLE 96)

4 f86les0

ISS 03000500010001010000000 e

2SS 210010 e

3\$S $47000000424700000077001 \mathrm{e}$

4SS-1 4700 e

S5S 24747001344450125011 foe

$t$

SBE $6.00120 \mathrm{~cm}$ diameter iron sphere

0010

rec $1-0.750001 .50000 .75$

sph 2000010

$\begin{array}{lllllll}\text { TpP } 3 & -300 & 300 & -300 & 300 & -200 & 200\end{array}$

sph 40000 le 4

end

sou +1

iron $+2-1$

air $+3-2$

voi $+4-3$

end

1111

$4 \mathrm{ro}$

1210000

0

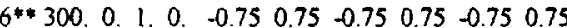

125\$ 11111122222

13\$\$ 130271200080161400050000

260566012250551503116032

$14^{* *} 1.349 \mathrm{e}-1 \quad 3.90 \mathrm{e}-3 \quad 2.60 \mathrm{e}-3 \quad 8.00 \mathrm{e}-4 \quad 4.00 \mathrm{e}-4$

8.475e-2 2.7621e-4 4.3133e-5 1.3771e-5 1.0346e-5 $17 * *$

$\begin{array}{lllll}1.77863 \mathrm{E}-04 & 4.97090 \mathrm{E}-04 & 2.24463 \mathrm{E}-03 & 4.33019 \mathrm{E}-03 & 8.47886 \mathrm{E}-03\end{array}$ $\begin{array}{llllll}2.12840 \mathrm{E}-02 & 3.66444 \mathrm{E}-02 & 8.78090 \mathrm{E}-02 & 7.79489 \mathrm{E}-02 & 4.31208 \mathrm{E}-02\end{array}$

$\begin{array}{lllll}4.49049 \mathrm{E}-02 & 1.90710 \mathrm{E}-02 & 3.82283 \mathrm{E}-03 & 2.30500 \mathrm{E}-02 & 6.92225 \mathrm{E}-02\end{array}$

6.76384E-02 8.46895E-02 $1.10074 \mathrm{E}-01 \quad 6.04808 \mathrm{E}-02 \quad 2.63742 \mathrm{E}-02$ 4.54912E-02 3.67124E-02 4.13280E-02 2.15884E-02 $3.10648 \mathrm{E}-02$ $1.64009 \mathrm{E}-02 \quad 8.06957 \mathrm{E}-03 \quad 3.90728 \mathrm{E}-03 \quad 1.10794 \mathrm{E}-03 \quad 6.34475 \mathrm{E}-04$ 1.93375E-04 2.26691E-04 6.04447E-04 5.43329E-04 $1.77137 \mathrm{E}-04$ 5.76214E-05 2.34756E-05 2.87325E-06 9.32885E-07 3.48420E-07 $\begin{array}{lllll}8.47274 \mathrm{E}-08 & 1.03648 \mathrm{E}-08 & 3.87084 \mathrm{E}-09 & 7.50834 \mathrm{E}-10 & 2.43763 \mathrm{E}-10\end{array}$ $1.03267 \mathrm{E}-10 \quad 1.39116 \mathrm{E}-11$

dose rate at specified location

dose rate, mrem/hr

flunce

19**

10800

15300

$20^{* *}$

f1. 0

21s5 $45 i 147$

end 
morse

SINDBAD SBE 6.001 Karlsruhe Iron Sphere Benchmark Experiment (BUGLE 80)

4 f861es0

1S\$0 $3000500010001010000000 e$

$2 \$ \$ 210010 \mathrm{e}$

$35 \$ 47000000424700000078001 \mathrm{e}$

$45 S-14700 \mathrm{e}$

SS\$ 24747001344450125011 foe

$t$

SBE 6.001 35cm diameter iron sphere

0010

$\begin{array}{llllllllll}\text { rce } 1 & -0.75 & 0 & 0 & 1.50 & 0 & 0 & 0.75\end{array}$

sph 20000017.5

$\begin{array}{llllllll}\text { rpp } & 3 & -300 & 300 & -300 & 300 & -200 & 200\end{array}$

sph 40000184

end

sou +1

iron $+2-1$

air $+3-2$

voi $+4-3$

end

1111

$4 \mathrm{ro}$

1210000

0

$\begin{array}{llllllllll}6 * * 300 & 0 & 1 & 0 & -0.75 & 0.75 & -0.75 & 0.75 & -0.75 & 0.75\end{array}$

I2\$\$ 1111122222

13\$\$ 130271200080161400050000

$2600060122505515031 \quad 16032$

$14 * 1.349 \mathrm{e}-1 \quad 3.90 \mathrm{e}-3 \quad 2.60 \mathrm{e}-3 \quad 8.00 \mathrm{e}-4 \quad 4.00 \mathrm{e}-4$

$17^{* *}$

$1.77863 \mathrm{E}-04 \quad 4.97090 \mathrm{E}-04 \quad 2.24463 \mathrm{E}-03 \quad 4.33019 \mathrm{E}-03 \quad 8.47886 \mathrm{E}-03$ $2.12840 \mathrm{E}-02 \quad 3.66444 \mathrm{E}-02 \quad 8.78090 \mathrm{E}-02 \quad 7.79489 \mathrm{E}-02 \quad 4.31208 \mathrm{E}-02$ $\begin{array}{llllll}4.49049 \mathrm{E}-02 & 1.90710 \mathrm{E}-02 & 3.82283 \mathrm{E}-03 & 2.30500 \mathrm{E}-02 & 6.92225 \mathrm{E}-02\end{array}$ $\begin{array}{llllll}6.76384 \mathrm{E}-02 & 8.46895 \mathrm{E}-02 & 1.10074 \mathrm{E}-01 & 6.04808 \mathrm{E}-02 & 2.63742 \mathrm{E}-02\end{array}$

$\begin{array}{llllll}4.54912 \mathrm{E}-02 & 3.67124 \mathrm{E}-02 & 4.13280 \mathrm{E}-02 & 2.15884 \mathrm{E}-02 & 3.10648 \mathrm{E}-02\end{array}$

$\begin{array}{lllll}1.64009 \mathrm{E}-02 & 8.06957 \mathrm{E}-03 & 3.90728 \mathrm{E}-03 & 1.10794 \mathrm{E}-03 & 6.34475 \mathrm{E}-04\end{array}$

1 93375E-04 2.26691E-04 604447E-04 5.43329E-04 1.77137E-04

$\begin{array}{llllll}5.76214 \mathrm{E}-05 & 2.34756 \mathrm{E}-05 & 2.87325 \mathrm{E}-06 & 9.32885 \mathrm{E}-07 & 3.48420 \mathrm{E}-07\end{array}$

$\begin{array}{llllll}8.47274 \mathrm{E}-08 & 1.03648 \mathrm{E}-08 & 3.87084 \mathrm{E}-09 & 7.50834 \mathrm{E}-10 & 2.43763 \mathrm{E}-10\end{array}$

$1.03267 \mathrm{E}-10 \quad 1.39116 \mathrm{E}-11$

$t$

dose rate at specified location

dose rate, mrem/hr

flunce

19**

10800

15300

$20^{* * *}$

f1. 0

21S\$ $45 i 147$

end 
BUGLE 96 VALIDATION WITH MORSE USING WATER

WSRC-TR-00349 AND IRON EXPERIMENTAL DATA FROM SINDBAD 97

Rev.0

Page 89 of 89

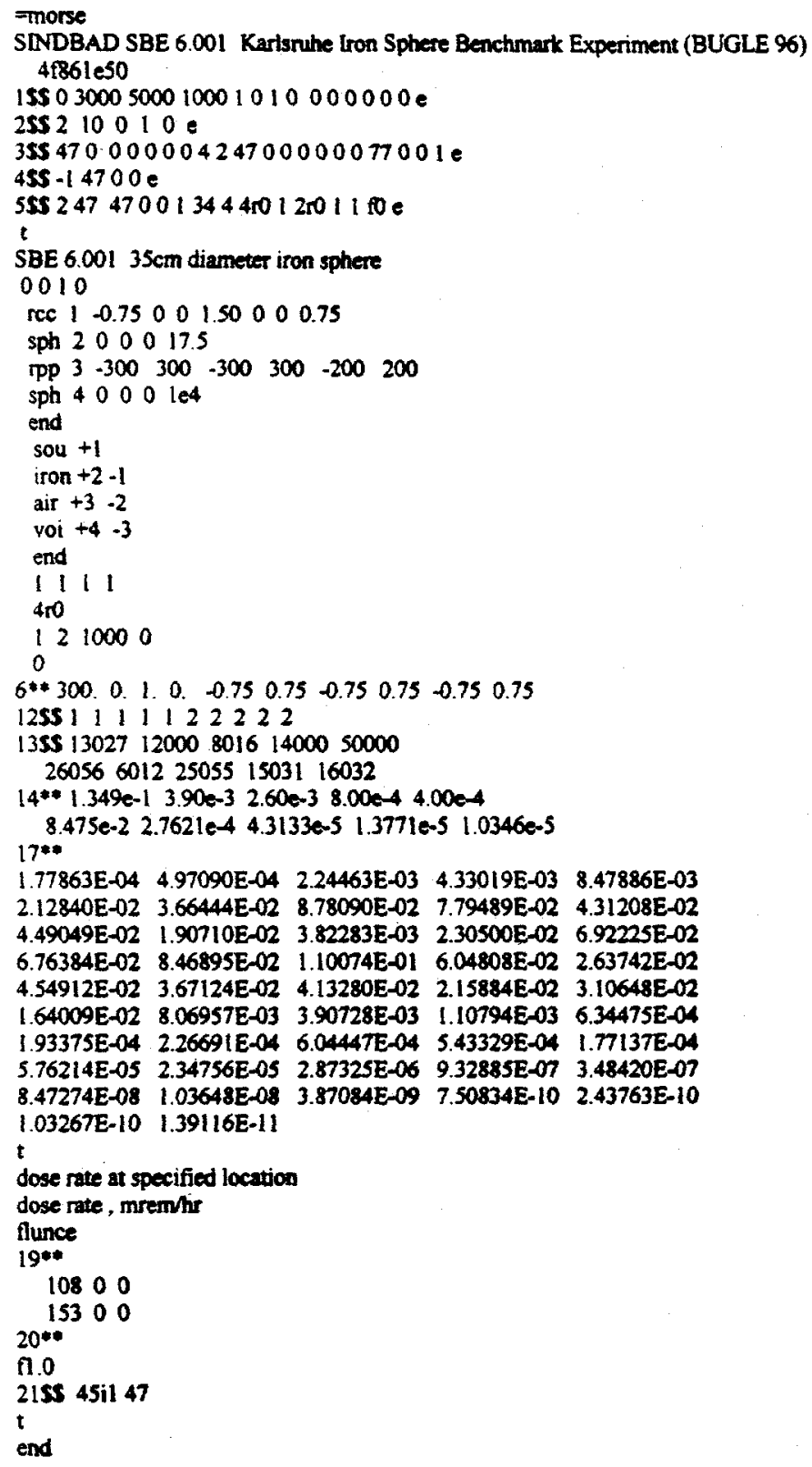

